\title{
Noncommutative localization in noncommutative geometry
}

\author{
ZORAN ŠKODA, email: zskoda@irb.hr \\ Institute Rudjer Bošković, P.O.Box 180, HR-10002 Zagreb, Croatia \\ - and - Institut des Hautes Études Scientifiques, \\ Le Bois-Marie, 35 route de Chartres, F-91440 Bures-sur-Yvette, France
}

February 8, 2020

\begin{abstract}
The aim of these notes is to collect and motivate the basic localization toolbox for the geometric study of "spaces", locally described by noncommutative rings and their categories of one-sided modules.

We present the basics of ORE localization of rings and modules in much detail. Common practical techniques are studied as well. We also describe a counterexample for a folklore test principle. Localization in negatively filtered rings arising in deformation theory is presented. A new notion of the differential ORE condition is introduced in the study of localization of differential calculi.

To aid the geometrical viewpoint, localization is studied with emphasis on descent formalism, flatness, abelian categories of quasicoherent sheaves and generalizations, and natural pairs of adjoint functors for sheaf and module categories. The key motivational theorems from the seminal works of GABRIEL on localization, abelian categories and schemes are quoted without proof, as well as the related statements of Popescu, Watts, Deligne and Rosenberg.

CoHn universal localization does not have good flatness properties, but may be described well at the ring level. We conjecture that the latter feature may be important for gluing in noncommutative geometry whenever the flat descent fails. Cohn localization is here related to the quasideterminants of GELFAND and RETAKH; and this may help understanding both subjects.
\end{abstract}




\section{Introduction}

1.1 Objectives and scope. This is an introduction to Ore localizations and generalizations, with geometric applications in mind.

The existing bibliography in localization theory is relatively vast, including several monographs. Localizations proliferate particularly in category theory, with flavours adapted to various situations, like bicategories, toposes, QuiLLEN's model categories, triangulated categories etc. A noncommutative algebraic geometer replaces a space with a ring or more general 'algebra', or with some category whose objects mimic modules over the 'algebra', or mimic sheaves over a space, or he/she studies a more general category which is glued from such local ingredients. This setup suggests that we may eventually need a similar toolbox to the ones used by the category and homotopy theorists; however the simplest cases are in the area of ring and module theory. Even so, we shift the emphasis from purely ring-theoretic questions to more geometrical ones.

A localized ring is typically structurally simpler than the original, but retaining some of its features. A useful tool for a ring theorist is a controlled but substantial simplification of a ring, often as extreme as passing to a local ring or a quotient skewfield. On the contrary, our main geometrical goal are those localizations which may play the role of noncommutative analogues of (rings of regular functions on principal Zariski) open sets in (say affine) variety. Rings of functions on these open sets may be slightly simpler than the rings of global functions, but not as radically as when, say, passing to a local ring. We start with the very basics of localization procedures. The geometric notion of a cover by localizations is studied in the noncommutative context as well. Only recent geometrically minded works include some elements of such study, as some key features of covers, the globalization lemma in particular, were recognized only in mid-eighties.

We use an elementary method to prove the existence and simple properties of the Ore localized rings, in line with the original 1931 O. ORE's paper [82] (who however assumed no zero divisors). Modern treatments often neglect details if sketching the elementary method. Another modern method (to prove existence etc. ([7])), following Asano (4]), is cheap, but does not give equivalent experience with the Ore method. Calculations similar to the ones in our proofs, appear in concrete examples of using or proving Ore conditions in practice. We also use this method to examine when there is an induced lo- 
calization of a first order differential calculus over a noncommutative ring, and come to a condition, not previously observed, which we call the "differential Ore condition". The elementary method has the advantage of being parallel to the calculus of (left) fractions in general categories, which has an aura of being a difficult subject, but it is more transparentafter learning Ore localization the elementary way.

Our next expositional goal is to obtain some practical criteria for finding and dealing with Ore localizations in practice. Folklore strategies to prove that some set is Ore are examined in detail. In a section on Ore localization in 'negatively' filtered rings, we explore similar methods.

While Ore localization is treated in a comprehensive introductory style, more general localizations are sketched in a survey style. For advanced topics there are often in place good references, assuming that the reader knows the motivation, and at least the Ore case. Both assumptions may be fulfilled by reading the present notes. We emphasize facts important in geometry, often omitted, or which are only a folklore. In order to clear up some sources of confusion, we sketch and compare several levels of generality; mention competing terminologies; and examine difficulties of geometrical interpretation and usage.

We focus on localizations of the category $R$ - Mod of all left modules over a fixed ring $R$. Localization in other specific categories, e.g. of 2-sided modules (symmetric localization, cf. [52]), bimodules, and the standard approach via injective hulls are omitted. In general, there are not enough 2-sided modules over a noncommutative ring, and bimodules are interpreted as corresponding to the maps of noncommutative rings, as explained in the text. Generalities on localization in arbitrary categories, and abelian in particular, are outlined for convenience.

As CoHn localization can be found in other works in this volume, we include only a short introduction with two goals: putting it in our context and, more importantly, relating it to the recent subject of quasideterminants. Anybody aware of both subjects is aware of some connection, and we try to spell it out as precisely as we can.

1.2 Prerequisites on algebraic structures. Basic notions on rings and modules are freely used: unital ring, left ideal, center, left module, bimodule, domain (ring with no zero divisors), skewfield (division ring), graded rings and modules, and operations with those.

1.3 Prerequisites on categories. The language of categories 
and functors is assumed, including a (universal) initial and terminal object, (projective/inverse) limit, colimit (= inductive/direct limit), (co)products, adjoint functors, Yoneda lemma and the categorical duality (inverting arrows, dual category, dual statements about categories).

1.4 A morphism $f: B \rightarrow C$ is mono (epi) if for any pair $e, e^{\prime}$ of morphisms from $C$ to any $D$ (resp. from any $A$ to $B$ ), equality $e f=e^{\prime} f$ (resp. $f e=f e^{\prime}$ ) may happen only if $e=e^{\prime}$.

A subobject is an equivalence class of monomorphisms. A pair $(F$, in) consisting of a functor $F$, and a natural transformation of functors in : $F \hookrightarrow G$, is a subfunctor of functor $G$, if all $\operatorname{in}_{M}: F(M) \hookrightarrow$ $G(M)$ are monomorphisms. Explicitly, for any $f: M \rightarrow N$ we have $\operatorname{in}_{N} \circ F(f)=G(f) \circ \operatorname{in}_{M}: F(M) \rightarrow G(N)$. Clearly, if $F$ is a subfunctor of an additive (k-linear) functor $G$, between additive (k-linear) categories, then $F$ is additive (k-linear) as well.

1.5 A (small) diagram $d$ in category $\mathcal{C}$ will be viewed as a functor from some (small) category $D$ into $\mathcal{C}$. For fixed $D$, such functors and natural transformations make a category $\mathcal{C}^{D}$. Every object $c$ in $\mathcal{C}$ gives rise to a constant diagram $c^{D}$ sending each $X$ in $D$ into $c$. This rule extends to a functor ()$^{D}: \mathcal{C} \rightarrow \mathcal{C}^{D}$. A cone over diagram $d: D \rightarrow \mathcal{C}$ is a natural transformation $I_{c}: c^{D} \Rightarrow d$ for some $c \in \mathcal{C}$. A morphism $I_{c} \rightarrow I_{c^{\prime}}$ of cones over $d$ is a morphism $\phi: c^{\prime} \rightarrow c$ such that $I_{c^{\prime}}=I_{c} \circ \phi^{D}$. A terminal among the cones over $D$ will be called a limiting cone over $D$. A colimiting cone in $\mathcal{C}$ is a limiting cone in opposite category $\mathcal{C}^{\text {op }}$. Consider a 'parallel' family of morphisms $\left\{f_{\gamma}: A \rightarrow B\right\}_{\gamma \in \Gamma}$ as a diagram $D$ with 2 objects and $|\Gamma|$ arrows in an obvious way. In this case, a cone over $D$ is given by a single map $I: c \rightarrow A$. We call the diagram $c \stackrel{I}{\rightarrow} A \stackrel{f_{\gamma}}{\Rightarrow} B$ a fork diagram. It is called an equalizer (diagram) if $I: c \rightarrow A$ is in addition a limiting cone; by abuse of language one often calls $I$, or even $c$ an equalizer. Equalizers in $\mathcal{C}^{\text {op }}$ are referred to as coequalizers. A morphism $I \rightarrow A$ of the cone of an equalizer diagram with $\Gamma=\{1,2\}$ is also called a kernel of parallel pair $f_{1}, f_{2}: A \rightarrow B$. Dually one defines cokernels.

A zero object 0 is an object which is simultaneously initial and terminal. In that case, composition $X \rightarrow 0 \rightarrow X$ is also denoted by $0: X \rightarrow X$. A (co)kernel of a single morphism $f: A \rightarrow B$ in a category with 0 is by definition a (co)kernel of pair $f, 0: A \rightarrow B$.

1.6 A functor $F: \mathcal{C} \rightarrow \mathcal{C}^{\prime}$ induces a (pullback) functor for diagrams $F \circ: \mathcal{C}^{D} \rightarrow\left(\mathcal{C}^{\prime}\right)^{D}$. It is defined by $d \mapsto F \circ d$ for every diagram $d: D \rightarrow \mathcal{C}$, and $\alpha \mapsto F(\alpha)$ where $(F(\alpha))_{M}:=F\left(\alpha_{M}\right)$ for $\alpha: d \Rightarrow d^{\prime}$. 
$F$ preserves limits of some class $\mathcal{P}$ of diagrams in $\mathcal{A}$ if it sends any limiting cone $p_{0} \rightarrow p$ over any $p \in \mathcal{P}$ in $\mathcal{A}$ into a limiting cone in $\mathcal{A}^{\prime}$. $F$ reflects limits if any cone $p_{0} \rightarrow p$ over any $p \in \mathcal{P}$ in $\mathcal{A}$ must be a limiting cone if $F$ sends it to a limiting cone in $\mathcal{A}^{\prime}$. The same with word limit replaced by colimit, and cone $p_{0} \rightarrow p$ by a cocone $p \rightarrow p_{0}$.

1.7 A preadditive category is a category $\mathcal{A}$ with an abelian group operation + on each set $\mathcal{A}(X, Y)$ such that each composition map $\circ: \mathcal{A}(X, Y) \times \mathcal{A}(Y, Z) \rightarrow \mathcal{A}(Y, Z)$ is bilinear. A preadditive category is additive if it contains a zero object and pairwise, hence all finite, products. Automatically then, finite products agree with finite coproducts. Recall that a preadditive category $\mathcal{A}$ is abelian if each morphism $f$ in $\mathcal{A}$ has a kernel and a cokernel morphism: and the kernel object of a cokernel equals the cokernel object of a kernel. We assume that the reader is comfortable with elementary notions on abelian groups like exact sequences and left (right) exact functors in the greater generality of abelian categories.

1.8 Gabriel-Mitchell-Popescu embedding theorem. Every small abelian category is equivalent as an abelian category to a subcategory of category of left modules over a certain ring R. Proof: [87.

1.9 Prerequisites on spaces of modern geometry. We expect familiarity with the notions of a presheaf, separated presheaf and sheaf, and with some examples describing a geometry via a topological space with a structure sheaf on it; as well as idea of gluing from some sort of local models, behind the concepts of (super)manifolds, analytic spaces and schemes. Earlier exposure to commutative algebraic (or analytic) varieties and schemes is assumed in style of some sections, but no specific facts are required; an abstract sketch of the main features of scheme-like theories is supplied in the text below.

1.10 Conventions. The word map means a set-theoretic map unless it is accompanied with a specification of the ambient category in question when it means a (homo)morphism, e.g. a map of rings means a ring (homo)morphism. The word noncommutative means "not necessarily commutative". We mostly deal with unital rings and modules, but each section has at least some labelling as regards to this assumption. Ideal without label means 1-sided (usually left) ideal.

The symbol for inclusion $\subset$ may include the case of equality. The unadorned tensor sign is over $\mathbb{Z}$, except for elements in known tensor products, like $a \otimes b:=a \otimes_{R} b \in A \otimes_{R} B$. For algebras and modules over a commutative ring, this ring is usually denoted $\mathbf{k}$. These conventions may be locally overwritten by contextual remarks. 


\section{Noncommutative geometry}

DESCARTES introduced the method of coordinates, which amounted to identification of real vector spaces with the space described by the axioms of EUCLID. LAGRANGE considered more general coordinates in analytic mechanics to obtain exhaustive treatments of space. Topological spaces do not have distinguished coordinate functions, but the whole algebra of functions suffices. The Gelfand-NEIMARK theorem stated that the category of compact Hausdorff topological spaces is equivalent to the opposite of category of commutative unital $C^{*}$ algebras. This is accomplished by assigning to a compact $X$ the Banach $\star$-algebra $C(X)$ of all continuous $\mathbb{C}$-valued functions (with supremum-norm and involution $f^{*}(x)=\overline{f(x)}$ ). In the other direction one (re)constructs $X$ as a Gelfand spectrum of the algebra $A$, which is a space whose points are continuous characters $\chi: A \rightarrow \mathbb{C}^{*}$ endowed with spectral topology. These characters appear as the evaluation functionals $\chi_{x}$ on $A$ at points $x \in X$, where $\chi_{x}(f)=f(x)$. Each annihilator Ann $\chi=\{a \in A \mid \chi(a)=0\}$ is a maximal ideal of $A$ and all maximal ideals are of that form.

2.1 Towards noncommutative algebraic geometry. For any commutative ring $R$, GROTHENDIECK replaced maximal ideals from the theory of affine varieties and from the Gelfand-Neimark picture, by arbitrary prime ideals, which behave better functorially, and he endowed the resulting spectrum $\operatorname{Spec} R$ with a non-Hausdorff Zariski topology and a structure sheaf, defined with the help of commutative localization. This amounts to a fully faithful contravariant functor Spec from Rings to the category $\mathfrak{i s p}$ of locally ringed spaces.

Statements about spaces in the images of Gelfand-Neimark and Spec-functors, may be in principle rephrased in terms of the initial algebras, and as such often make sense for noncommutative rings. Noncommutative geometry means exploring the idea of faithfully extending the Gelfand and Spec functors to noncommutative algebras as a domain and some geometrical universe $\mathfrak{n} \mathfrak{S} \mathfrak{s}$ generalizing category $\mathfrak{I S p}$ as a target, and thinking geometrically of the consequences of such construction(s). The category $\mathfrak{n l} \mathfrak{S p}$ should ideally contain more general noncommutative schemes, extending the fact that spaces in $\mathfrak{r} \mathfrak{S p}$ have topological base, enabling one to glue affine schemes over such subsets. This is as needed as in the commutative case where basic useful operations like blow-up, quotients by actions of groups etc. take us out of the realm of affine schemes. SoIBELman (110]) advocates 
examples of natural compactifications of moduli spaces of commutative spaces with noncommutative spaces as points on the boundary. There are also candidates for noncommutative Proj-functor on some category of graded rings (non-faithful as usual Proj).

But there is no fully satisfactory extension of the Spec-functor: some candidates are not faithful, yet others solve the problem formally, but do not really geometrize at least those rings which are very big and very noncommutative. Thus we fix some intermediate "semilocal" category slSp with a fully faithful contravariant functor Rings $\rightarrow$ slSp, and work in slSp. It is intermediate in the sense that it is still equivalent to the original but its features are yet not manifestly of a local and geometric nature. As such it does not extend commutative $\mathfrak{r} \mathfrak{s}$, but heuristically it is viewed as equivalent to the hypothetical $\mathfrak{n l} \mathfrak{S p}$ extension of $\mathfrak{r} \mathfrak{S p}$ which is geometric.

For special purposes we know many useful constructions, partially replacing the missing equivalence $\mathbf{s l S p} \rightarrow \mathfrak{n}$ lsp. We may think of such constructions as non-faithful functors $\mathbf{s l S p} \rightarrow \mathfrak{n l} \mathfrak{S p}_{\alpha}$ into some geometric realms $\mathfrak{n l} \mathfrak{S p}_{\alpha}$.

2.2 An example of slSp is as follows. Consider the center $Z(R)$ and construct commutative $\operatorname{Spec} Z(R)$. Then for each principal open set $U$ in $Z(R)$, one localizes $R$ at $U$ (a commutative localization) and this essentially gives the structure sheaf $U \rightarrow R_{U}=\mathcal{O}(U)$. The problem is, what if the center is small, hence $\operatorname{Spec} Z(R)$ as well: all the information on $R$ is kept in a few, maybe one $\operatorname{ring} R_{U}$, and we did not get far. In fact, taking the center is not functorial, so we have to modify the categories a bit, to allow for pairs $\left(R, Z^{\prime}\right), Z^{\prime} \subset Z(R)$ from the start. In some cases the base space $\operatorname{Spec} Z^{\prime}$ is big enough and we may glue such spectra to interesting more general "schemes".

A method which is sometimes useful is to add some other localizations, $Q_{i} R$, and think of them as structure rings $R_{U_{i}}$ over open subsets $U_{i}$. However, now $U_{i}$ is not a subset in $\operatorname{Spec} Z(R)$, but rather a functor $Q_{i}$ on the category of (left) $R$-modules.

2.3 MAnin has suggested (75]) a functor from graded rings into abelian categories: to a Noetherian ring $R$ assign the quotient of the category of finitely generated graded $R$-modules by the subcategory of the finitely generated graded $R$-modules of finite length. In the commutative case, by a theorem of SERRE, this category is equivalent to the category of coherent sheaves over Proj $R$. This is one of the candidates for projective noncommutative geometry and we view it as an example of functor of type slSp. Manin here follows 
GrothendiECK's advice that to do geometry one does not need the space itself but only the category of sheaves on that would-be space. In this spirit, Grothendieck defined topos (53] and [17, vol.3) as an abstract category satisfying a list of axioms, whose consequence is that it is equivalent to the category of sheaves Fas $\mathcal{C}$ over some site $\mathcal{C}$ (a category with a Grothendieck topology). Two different sites may give rise to the same topos, but their cohomological behaviour will be the same. Thus they are thought of as the same generalized space. In algebraic geometry we have parallel examples for the same heuristics, where we take the abelian categories of quasicoherent sheaves of $\mathcal{O}$ modules, instead of a topos of all sheaves of sets. Suitable morphisms between the topoi are recognized to be 'geometrical morphisms' which are also certain adjoint pairs; Grothendieck actually utilizes an interplay ('yoga') of 6 standard functors in topos theory. We discuss the basic pairs of adjoint functors for modules and sheaves. They appear in the disguise of maps of (noncommutative) rings (affine maps and their abstract version), as bimodules for two rings, as direct and inverse maps for $\mathcal{O}_{X}$-modules, and as localization functors.

2.4 Grothendieck categories (G.c.) [52, 109]. A Grothendieck category is a cocomplete (having all small limits) abelian category, with enough injectives and a small generator. The category of left $R$-modules, and the category of all sheaves of left $R$-modules over a fixed topological space, are G.c.'s. Given a coalgebra $C$, the category of $C$-comodules is G.c. Given a bialgebra $B$ and a $B$-comodule algebra $E$, the category of relative $(E, B)$-Hopf modules is a G. c. 128 .

Theorem. (P. GABRIEL) The category $\mathfrak{Q} \mathfrak{c o h}{ }_{X}$ of quasicoherent sheaves of $\mathcal{O}_{X}$-modules over a quasicompact quasiseparated scheme $X$ is a Grothendieck category.

It is not known if $\mathfrak{Q c o h}_{X}$ where $X$ is a general scheme is cocomplete nor if it has enough injectives. This fact is behind our decision not to strictly require our abelian categories of noncommutative geometry to be a G.c. (which is fashionable). Rosenberg 93 requires the weaker property (sup) (= categories with exact limits) introduced by GABRIEL (34]): for any object $M$ and any ascending chain of subobjects there is a supremum subobject, and taking such supremums commutes with taking the join (minimum) with a fixed subobject $N \subset M$. This holds for $R$-mod, Fas $\mathcal{C}$ (for a small site $\mathcal{C}$ ) and $\mathfrak{Q} \mathfrak{c o h}_{X}$ (for any scheme $X$ ).

2.5 Theorem. (P. Gabriel for noetherian schemes (34, Ch. $\mathrm{VI})$; A.L. RosenberG in quasicompact case (92]); and in general case (99])) Every scheme $X$ can be reconstructed from the abelian 
category $\mathfrak{Q c o h}_{X}$ uniquely up to an isomorphism of schemes.

This motivates the promotion to a "space" any member of a class of abelian categories, possibly satisfying some additional axioms, which must allow for (some variant) of $R$-mod and $\mathfrak{Q} \mathfrak{c o h}{ }_{X}$ as prime examples. A distinguished object $\mathcal{O}$ in $\mathcal{A}$, corresponding to the structure sheaf is often useful part of a data, even at the abstract level, hence the spaces could be actually pairs $(\mathcal{A}, \mathcal{O})$. The consideration of functors for categories of modules and categories of sheaves suggests there a special role for functors having various exactness properties (93]), e.g. having a right adjoint. Gluing categories over localizations, and variants thereof, should be interpreted in good cases as gluings of spaces from local models. In noncommutative geometry a local model is the category of modules over a noncommutative ring.

2.6 The so-called derived algebraic geometry, treating in more natural terms and globalizing the infinitesimal picture of moduli spaces governed by the deformation theory, appeared recently ([9, 116]). Its cousin, homotopical algebraic geometry appeared promising in the study of homotopy theories for algebraic varieties, and also in using the reasoning of algebraic geometry for ring spectra of homotopy theory and their globalization. In similar generalizations of algebraic geometry the basic gadgets are higher categories (e.g. simplicially enriched, DG, Segal, $A_{\infty}$, cf. [29, 89, 116, 117]). The lack of smoothness in some examples of moduli spaces is now explained as an artifact of the truncation process replacing the natural and smooth 'derived moduli spaces' by ordinary moduli spaces ('hidden smoothness principle' due Deligne, Drinfeld, Kapranov, Hinich...).

Part of the relevant structure here may be already expressed by replacing rings by differential graded algebras (dga-s) (9]), or, more generally, by introducing sequences of higher (e.g. 'MASSEY') products, as in the theory of $A_{\infty}$ (strongly homotopy associative) and $L_{\infty}$ (strongly homotopy Lie) algebras. Such generalizations and special requirements needed to do localization in such enriched settings, are beyond the scope of the present article. A noncommutative algebraic geometry framework designed by O.A. LAUDAL (63), with emphasis on the problem of noncommutative deformation of moduli, implicitly includes the higher Massey products as well. In the viewpoint put forward by KONTSEVICH and FUKAYA, some of the 'dualities' of the modern mathematical physics, e.g. the homological mirror symmetry, involve certain $A_{\infty}$-categories defined in terms of geometric data $([58,174,110])$. The so-called quantization $([26$, 56, 62, 130]) 
in its many versions is generally of deformational and noncommutative nature. Thus it is not surprising that the formalisms combining the noncommutative and homological (or even homotopical) structures benefit from the geometrically sound models of quantum physics. MANIN suggested that a more systematic content of a similar nature exists, programmatically named quantized algebraic geometry, and that it may shed light on hidden aspects of the geometry of (commutative) varieties, including the deep subject of motives.

An interesting interplay of derived categories of coherent sheaves on varieties and their close analogues among other triangulated categories, motivates some 'noncommutative' geometry of the latter (13. [12]). Triangulated categories are also only a truncation of some other higher categories.

One should also mention that some important classes of rings in quantum algebra, for example quantum groups, may be constructed using categories of (perverse) sheaves over certain commutative configuration spaces $(70)$. Thus the structure of various sheaf categories is an ever repeating theme which relates the commutative and noncommutative world. See the essay [22] for further motivation.

\section{Abstract localization}

We discuss localization of 1 . algebraic structures; 2 . categories. These two types are related: typically a localization of a ring $R$ induces a localization of the category $R$ - Mod of left modules over $R$.

A recipe $\mathcal{G}$ for a localization takes as input a structure $R$ (monoid, lattice, ring), or a category $\mathcal{A}$, and a distinguished data $\Sigma$ in $R$ (or $\mathcal{A}$ ). The localizing data $\Sigma$ is selected from some class $\mathcal{U}(R)$ of structural data, for example elements, endomorphisms or ideals of $R$; similarly $\mathcal{U}(\mathcal{A})$ could be a class of subcategories or collection of morphisms in $\mathcal{A}$. Usually not all obvious subclasses of $\mathcal{U}(R)$ may serve as distinguished data for $\mathcal{G}$, and some 'localizability' conditions apply.

A localization procedure $\mathcal{G}(R, \Sigma)$ should replace $R$ by another object $Y$ and a map $i: R \rightarrow Y$, which induces, for given $\mathcal{G}$, some canonical correspondence $\mathcal{G}(i): \Sigma \rightsquigarrow \Sigma_{*}$ between the localization data $\Sigma$ and some other data $\Sigma_{*}$ chosen from $\mathcal{U}(Y)$. The subclass $\Sigma_{*}$ should satisfy some natural requirement, for example that it consist of invertible elements. Pair $(i, Y)$ should be in some sense minimal, or universal among all candidates satisfying the given requirements. For given re- 
quirements only certain collections $\Sigma$ built from elements in $\mathcal{U}(R)$ give rise to a universal $(i, Y)$. Such $\Sigma$ are generically called localizable and the resulting $Y$ is denoted $\Sigma^{-1} R$.

In the case of a category $\mathcal{C}$, a map $i$ is replaced by a localization functor $Q^{*}: \mathcal{C} \rightarrow \Sigma^{-1} \mathcal{C}$. In this article, a localization of a category will be equivalent to an abstract localization with respect to a class of morphisms $\Sigma$ in $\mathcal{C}$, often using some other equivalent data (e.g. 'localizing subcategory'). Following [35], we sketch the general case of a localization at a class of morphisms $\Sigma$, cf. also [17.

3.1 An abstract 1-diagram $\mathcal{E}$ is a structure weaker then a category: it consists of a class $\mathrm{Ob} \mathcal{E}$ of objects and a class Mor $\mathcal{E}$ of morphisms equipped with a source and a target maps $\mathrm{s}$, T $:$ Mor $\mathcal{E} \rightarrow \mathrm{Ob} \mathcal{E}$. No composition, or identity morphisms are supplied. As usual, for two objects $A, B$ by $\mathcal{E}(A, B)$ we denote class of morphisms $f$ with $\mathrm{s}(f)=A$ and $\mathrm{T}(f)=B$. If each $\mathcal{E}(A, B)$ is a set, one may use word (multiple-edge) graph instead. If $\mathcal{E}, \mathcal{C}$ are diagrams, an $\mathcal{E}$-diagram in $\mathcal{C}$, or a morphism $d: \mathcal{E} \rightarrow \mathcal{C}$, is any pair of maps $\mathrm{Ob} \mathcal{E} \rightarrow \mathrm{Ob} \mathcal{C}$ and $\operatorname{Mor} \mathcal{E} \rightarrow \operatorname{Mor} \mathcal{C}$ which commute with source and target maps. Small abstract 1-diagrams and their morphisms form a category $\mathfrak{D i a g r}_{1}$. To each category one assigns its underlying abstract diagram. This correspondence induces a forgetful functor from the category $\mathfrak{C} a t$ of small categories to $\mathfrak{D i a g r}_{1}$. The construction of a category of paths below provides the left adjoint to this functor.

If $n \geq 0$ is an integer, a path of length $n$ from $A$ to $B$ in an abstract diagram $\mathcal{E}$ is a tuple $\left(A, f_{1}, f_{2}, \ldots, f_{n}, B\right)$, where $A$ is an object and $f_{i}$ are morphisms in $\mathcal{E}$, such that $\mathrm{s}\left(f_{i+1}\right)=\mathrm{T}\left(f_{i}\right)$ for $i=1, \ldots, n-1$, and $\mathrm{s}\left(f_{1}\right)=A, \mathrm{~T}\left(f_{n}\right)=B$ if $n>0$, and $A=B$ if $n=0$. For any abstract 1-diagram $\mathcal{E}$ define a category $\mathrm{Pa} \mathcal{E}$ of paths in $\mathcal{E}$ as follows. The class of objects is

$$
\mathrm{ObPa} \mathcal{E}:=\operatorname{Ob} \mathcal{E}
$$

and the morphism class $(\mathrm{Pa} \mathcal{E})(A, B)$ consists of all paths from $A$ to $B$. One declares $\operatorname{Id}_{A}:=(A, A), \mathrm{s}^{\prime}\left(A, f_{1}, \ldots, f_{n}, B\right)=A$ and $\mathrm{T}^{\prime}\left(A, f_{1}, \ldots, f_{n}, B\right)=B$ to be identity morphisms, and source and target maps for $\operatorname{Pa} \mathcal{E}(A, B)$. The composition rule is

$$
\left(A, f_{1}, \ldots, f_{n}, B\right) \circ\left(B, g_{1}, \ldots, g_{m}, C\right)=\left(A, f_{1}, \ldots, f_{n}, g_{1}, \ldots, g_{m}, C\right) .
$$

If each $(\mathrm{Pa} \mathcal{E})(A, B)$ is small we indeed obtain a category.

Consider the canonical $\mathcal{E}$-diagram $i_{\mathcal{E}}: \mathcal{E} \rightarrow \mathrm{Pa} \mathcal{E}$ which is tautological on objects as well as on paths of length 1 . $\mathrm{Pa} \mathcal{E}$ has the following 
universal property: an $\mathcal{E}$-diagram $d$ in any category $\mathcal{C}$ gives rise to a unique functor $d^{\prime}: \mathrm{Pa} \mathcal{E} \rightarrow \mathcal{C}$ such that $d=d^{\prime} \circ i_{\mathcal{E}}$.

3.2 Let $\Sigma$ be a family of morphisms in category $\mathcal{C}$. If $J: \mathcal{C} \rightarrow \mathcal{D}$ is any functor let $\Sigma_{*}:=J(\Sigma)$ be the class of all morphisms $J(f)$ where $f \in \Sigma$. Given $\mathcal{C}$ and $\Sigma$, consider the diagram scheme $\mathcal{E}=\mathcal{E}(\mathcal{C}, \Sigma)$ with $\operatorname{Ob} \mathcal{E}:=\operatorname{Ob} \mathcal{C}$ and Mor $\mathcal{E}:=\operatorname{Mor} \mathcal{C} \coprod \Sigma, \mathrm{s}_{\mathcal{E}}=\left.\mathrm{s} \coprod \mathrm{s}\right|_{\Sigma}, \mathrm{T}_{\mathcal{E}}=\left.\mathrm{T} \coprod \mathrm{T}\right|_{\Sigma}$. One has canonical inclusions $\mathrm{IN}_{1}: \operatorname{Mor} \mathcal{C} \rightarrow \operatorname{Mor} \mathcal{E}, \mathrm{IN}_{2}: \Sigma \hookrightarrow \operatorname{Mor} \mathcal{E}$. Let $\sim$ be the smallest equivalence relation on $\mathrm{Pa} \mathcal{E}$ such that

$$
\begin{aligned}
& \left(\mathrm{IN}_{1} v\right) \circ\left(\mathrm{IN}_{1} u\right) \sim \operatorname{IN}_{1}(v \circ u) \text { if } v \circ u \text { is defined in } \mathcal{C}, \\
& \operatorname{IN}_{1}\left(\operatorname{id}_{A}\right) \sim(A, A), \quad A \in \mathcal{C}, \\
& \left.\begin{array}{l}
\left(\mathrm{IN}_{2} f\right) \circ\left(\mathrm{IN}_{1} f\right) \sim(\mathrm{S}(f), \mathrm{S}(f)) \\
\left(\mathrm{IN}_{1} f\right) \circ\left(\mathrm{IN}_{2} f\right) \sim(\mathrm{T}(f), \mathrm{T}(f))
\end{array}\right\} f \in \Sigma .
\end{aligned}
$$

It is direct to show that operation $\circ$ induces a composition on classes of morphisms with respect to this particular equivalence relation. In this way we obtain a quotient $\Sigma^{-1} \mathcal{C}$ of the category $\mathrm{Pa} \mathcal{E}$ together with the canonical functor $Q_{\Sigma}^{*}: \mathcal{C} \rightarrow \mathrm{Pa} \mathcal{E}$ which is tautological on objects and equals $i_{\mathcal{E}} \circ \mathrm{IN}_{1}$ followed by the projection to the classes of equivalence on morphisms.

3.3 Proposition. If $f \in \Sigma$ then the functor $Q_{\Sigma}^{*}: \mathcal{C} \rightarrow \Sigma^{-1} \mathcal{C}$ sends $f$ to an invertible map $Q_{\Sigma}^{*}(f)$. If $T: \mathcal{C} \rightarrow \mathcal{D}$ is any functor such that $T(s)$ is invertible for any $s \in \Sigma$ then there is a unique functor $H: \Sigma^{-1} \mathcal{A} \rightarrow \mathcal{B}$ such that $T=H \circ Q_{\Sigma}^{*}$.

$\Sigma^{-1} \mathcal{C}$ is the category of fractions of $\mathcal{C}$ at $\Sigma$. This construction has a defect, in that there is no general recipe to determine when two morphisms in $\mathrm{Pa} \mathcal{E}$ represent the same morphism in $\Sigma^{-1} \mathcal{C}$. If $\Sigma$ satisfies the Ore conditions, below, there is one.

3.4 Proposition. 35] Let $T^{*} \dashv T_{*}$ be an adjoint pair of functors (this notation means that $T^{*}$ is left adjoint to $T_{*}$ ), where $T^{*}: \mathcal{A} \rightarrow \mathcal{B}$. with adjunction counit $\epsilon: T^{*} T_{*} \Rightarrow 1_{\mathcal{B}}$. Let $\Sigma=\Sigma\left(T_{*}\right)$ be the class of all morphisms $f$ in $\mathcal{A}$ such that $T^{*}(f)$ is invertible, and $Q^{*}: \mathcal{A} \rightarrow \Sigma^{-1} \mathcal{A}$ the natural functor. Then the following are equivalent:

(i) $T_{*}$ is fully faithful.

(ii) $\epsilon: T^{*} T_{*} \Rightarrow 1_{\mathcal{B}}$ is an isomorphism of functors.

(iii) The unique functor $H: \Sigma^{-1} \mathcal{A} \rightarrow \mathcal{B}$ such that $T^{*}=H \circ Q_{\Sigma}^{*}$ is an equivalence; in particular $Q_{\Sigma}^{*}$ has a right adjoint $Q_{\Sigma *}$.

(iv) (If $\mathcal{A}$ is small) For each category $\mathcal{X}$, functor $\mathfrak{C a t}(-, \mathcal{X})$ : $\mathfrak{C a t}(\mathcal{B}, \mathcal{X}) \rightarrow \mathfrak{C a t}(\mathcal{A}, \mathcal{X})$ is fully faithful.

Throughout the paper, any functor $T^{*}$ satisfying (i)-(iii) will be referred to as a continuous localization functor. 
3.5 66] Suppose $Q^{*}: \mathcal{A} \rightarrow \mathcal{B}$ is a continuous localization functor (cf. 3.4), and $F: \mathcal{A} \rightarrow \mathcal{A}$ an endofunctor. If there is a functor $G: \mathcal{B} \rightarrow \mathcal{B}$ and a natural equivalence of functors $\alpha: Q^{*} \circ F \Rightarrow G \circ Q^{*}$ then there is a unique functor $F_{\mathcal{B}}: \mathcal{B} \rightarrow \mathcal{B}$ such that $Q^{*} \circ F=F_{\mathcal{B}} \circ Q^{*}$. In that case, we say that $F$ is compatible with $Q^{*}$.

Proof. Suppose $f: M \rightarrow N$ is a morphism in $\mathcal{A}$. Suppose that $Q^{*}(f)$ is invertible. Then $G Q^{*}(f): G Q^{*}(M) \rightarrow G Q^{*}(N)$ also has some inverse $s$. The naturality of $\alpha$ and $\alpha^{-1}$ implies

$$
\begin{aligned}
& \alpha_{M}^{-1} \circ s \circ \alpha_{N} \circ Q^{*} F(f)=\alpha_{M}^{-1} \circ s \circ G Q^{*}(f) \circ \alpha_{M}=\operatorname{id}_{M}, \\
& Q^{*} F(f) \circ \alpha_{M}^{-1} \circ s \circ \alpha_{N}=\alpha_{N}^{-1} \circ G Q^{*}(f) \circ s \circ \alpha_{N}=\operatorname{id}_{N},
\end{aligned}
$$

hence $\alpha_{M}^{-1} \circ s \circ \alpha_{N}: Q^{*} F(N) \rightarrow Q^{*} F(M)$ is the inverse of $Q^{*} F(f)$. The conclusion is that for any $f$ with $Q^{*}(f)$ invertible, $Q^{*} F(f)$ is invertible as well. In other words, (by the universal property of the localization), functor $Q^{*} F$ factors through the quotient category $\mathcal{B}$, i.e. $\exists$ ! $F_{\mathcal{B}}: \mathcal{B} \rightarrow \mathcal{B}$ with $Q^{*} \circ F=F_{\mathcal{B}} \circ Q^{*}$. Q.E.D.

\section{Ore localization for monoids}

4.1 A semigroup is a set $R$ with a binary associative operation. A semigroup with unit element $1 \in R$ is called a monoid. By definition, maps of semigroups are set maps which respect the multiplication, and maps of monoids should preserve unit element as well. Monoids and maps of monoids form a category $\mathfrak{M o n}$, which has arbitrary products. The notion of a submonoid is the obvious one.

A subset $S$ of a monoid $R$ is multiplicative if $1 \in S$ and whenever $s_{1}, s_{2} \in S$ then $s_{1} s_{2} \in S$. For a set $S_{1} \subset R$ there is a smallest multiplicative subset $S \subset R$ containing $S_{1}$, namely the set of all products $s_{1} \cdots s_{n}$ where $s_{i} \in S_{1}$, including the product of the empty set of elements which equals 1 by definition. We say that $S$ is multiplicatively generated by $S_{1}$.

4.2 A multiplicative subset $S \subset R$ is a left Ore set if

- $\left(\forall s \in S \forall r \in R \exists s^{\prime} \in S \exists r^{\prime} \in R\right)\left(r^{\prime} s=s^{\prime} r\right)$ (left Ore condition);

- $\left(\forall n_{1}, n_{2} \in R \forall s \in S\right)\left(n_{1} s=n_{2} s\right) \Rightarrow\left(\exists s^{\prime} \in S, s^{\prime} n_{1}=s^{\prime} n_{2}\right)$ (left reversibility).

4.3 In traditional ring-theoretic terminology, $S$ is a left Ore set if the first condition holds and $S$ is a left denominator set if both 
conditions hold. We often say "left Ore set" for a left denominator set, as is increasingly common among geometers, and the notion of satisfying just the left Ore condition may be said simply "satisfying left Ore condition". By saying (plural:) "left Ore conditions" we subsume both the left Ore condition and the left reversibility.

4.4 A monoid $R$ can be viewed as a small category $\operatorname{Cat}(R)$ with a single object $R$. Left multiplication by an element $a \in R$ is a morphism in Cat $R$ denoted by $L_{a}$. We compose the morphisms by composing the maps. Any small category having one single object is clearly equivalent to $\operatorname{Cat}(R)$ for a suitable $R$.

This suggests a generalization of the notion of a denominator set (as well as its applications below) by replacing Cat $(R)$ by an arbitrary category. A multiplicative system in a category $\mathcal{A}$ is a class $\Sigma$ of morphisms in $\mathcal{A}$ where all identity morphisms $1_{A}$, where $A \in \operatorname{Ob} \mathcal{A}$, are in $\Sigma$, and for any two composable morphism $s, t \in \Sigma$ (i.e., the target (range) of $t$ matches the source (domain) of $s$ ), also $s \circ t \in S$.

A multiplicative system $\Sigma$ satisfies the left Ore conditions if it satisfies the ordinary left Ore condition with all quantifiers conditioned on matching of the source and target maps appropriately.

More precisely, $\Sigma$ satisfies the left Ore condition if

$$
\forall(s: A \rightarrow B) \in \Sigma, \forall r: A^{\prime} \rightarrow B, \exists\left(s^{\prime}: D \rightarrow A^{\prime}\right) \in \Sigma, \exists r^{\prime}: D \rightarrow A,
$$

so that $r^{\prime} \circ s=s^{\prime} \circ r$. $S$ satisfies left reversibility ('simplifiability') if

$$
\begin{gathered}
\left(\forall n_{1}, n_{2}: A \rightarrow B, \forall(s: C \rightarrow A) \in \Sigma\right)\left(n_{1} \circ s=n_{2} \circ s\right) \\
\Rightarrow\left(\exists\left(s^{\prime}: B \rightarrow D\right) \in \Sigma, s^{\prime} \circ n_{1}=s^{\prime} \circ n_{2}\right) .
\end{gathered}
$$

We may picture the left simplifiability by the diagram

$$
C \stackrel{s}{\longrightarrow} A \underset{n_{2}}{\stackrel{n_{1}}{\longrightarrow}} B \stackrel{s^{\prime}}{\rightarrow} D
$$

We say that $\Sigma$ is a left denominator system, or equivalently, that the pair $(\mathcal{A}, \Sigma)$ forms a left calculus of fractions if the left Ore and left simplifiability condition hold. The book 88, has a good graphical treatment of that subject. See also [17, 30, 35, 87.

4.5 Lemma. Let $f: R \rightarrow R^{\prime}$ be a surjective map of monoids and $S \subset R$ left Ore. Then $f(S)$ is left Ore in $R^{\prime}$.

4.6 Let $\mathcal{D}$ be some category of monoids with additional structure, i.e. a category with a forgetful functor $(-)_{\text {mon }}: \mathcal{D} \rightarrow \mathfrak{M o n}$. If $R$ is an 
object in $\mathcal{D}$, a multiplicative set in $R$ is by definition any multiplicative set $S \subset(R)_{\text {mon }}$.

Definition. Given a multiplicative set $S$ in $R \in \mathcal{D}$ we introduce category $\mathcal{C}_{\mathcal{D}}(R, S)$ as follows. The objects of $\mathcal{C}_{\mathcal{D}}(R, S)$ are all pairs $(j, Y)$ where $Y \in \mathrm{Ob} \mathcal{D}$ and $j: R \rightarrow Y$ is a morphism in $\mathcal{D}$ satisfying

- $(\forall s \in S)(\exists u \in Y)(u j(s)=j(s) u=1)$ in $(Y)_{\operatorname{mon}}$;

The morphisms of pairs $\sigma:(j, Y) \rightarrow\left(j^{\prime}, Y^{\prime}\right)$ are precisely those morphisms $\sigma: Y \rightarrow Y^{\prime}$ in $\mathcal{D}$, for which $\sigma \circ j=j^{\prime}$.

In plain words, we consider those morphisms which invert all $s \in S$.

Now we would like multiplying $j\left(s_{1}\right)^{-1} j\left(r_{1}\right) \cdot j\left(s_{2}\right)^{-1} j\left(r_{2}\right)$ to obtain again a 'left fraction' $j(s)^{-1} j(r)$. For this it is enough to be able to 'commute' the two middle terms in the sense $j\left(r_{1}\right) j\left(s_{2}\right)^{-1}=$ $j\left(s^{\prime}\right)^{-1} j\left(r^{\prime}\right)$ as $\left.j\left(s_{1}\right)^{-1} j\left(s^{\prime}\right)^{-1}\right)=j\left(s^{\prime} s_{1}\right)^{-1}$ and $j\left(r^{\prime}\right) j\left(r_{2}\right)=j\left(r^{\prime} r_{2}\right)$ and we are done. This reasoning is the origin of the left Ore condition. Here is a formal statement:

4.7 Proposition. (i) For $(j, Y) \in \mathrm{Ob} \in \mathcal{C}_{\mathfrak{M o n}}(R, S), j(S)$ is left Ore in $j(R)$ iff

$$
j(S)^{-1} j(R)=\left\{j(s)^{-1} j(r) \mid s \in S, r \in R\right\} \subset Y
$$

is a submonoid of $Y$. In particular, if $S$ is left Ore in $R, j(S)^{-1} j(R)$ is a submonoid of $Y$ for each $(j, Y) \in \mathrm{Ob}(\mathcal{C}(R, S))$.

(ii) If the equivalent conditions in (i) hold, then

$$
\begin{gathered}
\forall(s, r) \in S \times R \\
j(s)^{-1} j(r)=j\left(s^{\prime}\right)^{-1} j\left(r^{\prime}\right)
\end{gathered} \Leftrightarrow\left\{\begin{array}{l}
\exists \tilde{s} \in S, \exists \tilde{r} \in R \\
j(\tilde{s}) j\left(s^{\prime}\right)=j(\tilde{r}) j(s) \\
j(\tilde{s}) j\left(r^{\prime}\right)=j(\tilde{r}) j(r) .
\end{array}\right.
$$

Proof. (i) $(\Rightarrow)$ Let $s_{1}, s_{2} \in S r_{1}, r_{2} \in R$. By the left Ore condition $\exists s^{\prime} \in S \exists r^{\prime} \in R$ with $j\left(s^{\prime}\right) j\left(r_{1}\right)=j\left(r^{\prime}\right) j\left(s_{2}\right)$. Hence the product $j\left(s_{1}\right)^{-1} j\left(r_{1}\right) \cdot j\left(s_{2}\right)^{-1} j\left(r_{2}\right)=j\left(s^{\prime} s_{1}\right)^{-1} j\left(r^{\prime} r_{2}\right)$ belongs to $Y$.

$(\Leftarrow)$ If $j(S)^{-1} j(R)$ is a monoid then $j(r) j(s)^{-1} \in j(S)^{-1} j(R)$. In other words, $\exists s^{\prime} \in S \exists r^{\prime} \in R$ such that $j(r) j(s)^{-1}=j\left(s^{\prime}\right)^{-1} j\left(r^{\prime}\right)$. Thus $j\left(s_{1}\right) j(r)=j\left(r_{1}\right) j(s)$.

(ii) By multiplying from the left by $j\left(s^{\prime}\right)$ one gets $j\left(s^{\prime}\right) j\left(s^{-1}\right) j(r)=$ $j\left(r^{\prime}\right)$. As $S$ is left Ore, $\exists \tilde{s} \in S \exists \tilde{r} \in R$ such that $\tilde{s} s^{\prime}=\tilde{r} s$. This implies $j(\tilde{s}) j\left(s^{\prime}\right)=j(\tilde{r}) j(s)$ and, consequently, $j\left(s^{\prime}\right) j\left(s^{-1}\right)=j(\tilde{s})^{-1} j(\tilde{r})$; then $j(\tilde{s})^{-1} j(\tilde{r}) j(r)=j\left(r^{\prime}\right)$ and, finally, $j(\tilde{r}) j(r)=j(\tilde{s}) j\left(r^{\prime}\right)$.

4.8 Proposition. Let $S, R, Y, j$ be as in 4.7, and let $R, Y$ be each equipped with a binary operation, in both cases denoted by $+_{0}$, 
such that - is left distributive with respect to $+_{0}$. If $j(S)^{-1} j(R)$ is a submonoid of $Y$, then it is closed with respect to $+_{0}$ as well.

Proof. The following calculation is valid in $Y$ :

$$
\begin{aligned}
j\left(s_{1}\right)^{-1} j\left(r_{1}\right)+ & j\left(s_{2}\right)^{-1} j\left(r_{2}\right)=j\left(s_{1}\right)^{-1}\left(j\left(r_{1}\right)+_{0} j\left(s_{1}\right) j\left(s_{2}\right)^{-1} j\left(r_{2}\right)\right) \\
& =j\left(s_{1}\right)^{-1}\left(j\left(r_{1}\right)+_{0} j(\tilde{s})^{-1} j(\tilde{r}) j\left(r_{2}\right)\right) \\
& =j\left(\tilde{s} s_{1}\right)^{-1}\left(j(\tilde{s}) j\left(r_{1}\right)+_{0} j(\tilde{r}) j\left(r_{2}\right)\right) \in j(S)^{-1} j(R),
\end{aligned}
$$

where $j(\tilde{s}) j\left(s_{1}\right)=j(\tilde{r}) j\left(s_{2}\right)$ for some $\tilde{s}, \tilde{r}$ by the left Ore condition which holds due 4.7.

4.9 Remark. We do not require $j\left(a++_{0} b\right)=j(a)+_{0} j(b)$ here.

4.9a Exercise. Generalize this to a family $\mathcal{F}$ of $n$-ary left distributive operations in place of $+_{0}$, i.e., of operations of the form $F: X^{\times n} \rightarrow X$, for various $n \geq 0$, such that $L_{a} \circ F=F \circ L_{a}^{\times n}$.

4.10 From now on we limit to the case where the category $\mathcal{D}$ above corresponds to a variety $\mathfrak{D}$ of algebras $\left(A, \mathcal{L}_{A}\right)$ (in the sense of universal algebra) of signature $\mathcal{L}=(\cdot, 1, \mathcal{F})$, where $\mathcal{F}_{A}$ is a family of left distributive operations on $A$ on a $\left(A, \cdot_{A}, 1_{A}\right)$. The reader who does not care for this generality (suitable say for algebras with operators) can consider 3 basic cases: 1) $\mathcal{F}=\emptyset$ when $\mathcal{D}=\mathfrak{M o n}$; 2) $\mathcal{F}=\{+\}$ and algebras are unital rings; 3$) \mathcal{F}=\{+\}$ and algebras are associative unital $\mathbf{k}$-algebras over a commutative ring $\mathbf{k}$.

4.11 Denote by $\mathcal{C}_{l, \mathcal{D}}(R, S)$ the full subcategory of $\mathcal{C}_{\mathcal{D}}(R, S)$ consisting of those objects $(j, Y)$ which satisfy

- $\left(\forall r, r^{\prime} \in R\right)\left(j(r)=j\left(r^{\prime}\right) \Leftrightarrow \exists s \in S\left(s r=s r^{\prime}\right)\right)$.

- $j(S)^{-1} j(R)$ is a subring of $Y$

Similarly, $\mathcal{C}_{l, \mathcal{D}}^{-}(R, S)$ by definition consists of objects satisfying the first, but not necessarily the second property. Denote by $\mathcal{C}_{r, \mathcal{D}}(R, S)$ the full subcategory of $\mathcal{C}_{\mathcal{D}}(R, S)$ consisting of those objects $(j, Y)$ which satisfy the symmetric conditions

- $\left(\forall r, r^{\prime} \in R\right)\left(j(r)=j\left(r^{\prime}\right) \Leftrightarrow \exists s \in S\left(r s=r^{\prime} s\right)\right)$.

- $j(R) j(S)^{-1}$ is a subring of $Y$

Finally, the objects in $\mathcal{C}_{r, \mathcal{D}}^{-}(R, S)$, by definition, satisfy the first, but not necessarily the second property. If there is a universal initial object in $\mathcal{C}_{l}(R, S)\left(\mathcal{C}_{r}(R, S)\right)$, we denote it by $\left(\iota, S^{-1} R\right)\left(\operatorname{resp} .\left(\iota, R S^{-1}\right)\right)$ and we call the pair, or by abuse of language, also the $\operatorname{ring} S^{-1} R\left(R S^{-1}\right.$ resp.), the left (right) Ore localization of $R$ at set $S$, and map $\iota$ the localization map. An alternative name for $S^{-1} R\left(R S^{-1}\right)$ is the left (right) ring of fractions (of ring $R$ at set $S$ ). 
4.12 Proposition. If for any $(j, Y)$ in $\mathcal{C}_{l, \mathfrak{M}_{\mathfrak{n}}}^{-}(R, S)$ the subset $j(S)^{-1} j(R)$ is a submonoid (i.e. $\exists(j, Y) \in \mathcal{C}_{l, \mathfrak{M} \text { on }}(R, S)$ ) then it is for every $(j, Y)$ in $\mathcal{C}_{l, \mathfrak{M o n}}^{-}(R, S)$, i.e. the categories $\mathcal{C}_{l, \mathfrak{M o n}}(R, S)$ and $\mathcal{C}_{l, \mathfrak{M o n}}^{-}(R, S)$ coincide. In that case, $S$ is a left denominator set in $R$.

Proof. Let $j(S)^{-1} j(R)$ be a subring for some $(j, Y)$. Then $j(S)^{-1} j(R)$ is Ore in $j(R)$ by the previous proposition. Thus for every $s \in S$, $r \in R \exists s^{\prime} \in S \exists r^{\prime} \in R$ such that $j(r) j(s)^{-1}=j\left(s^{\prime}\right)^{-1} j\left(r^{\prime}\right)$ and therefore $j\left(s^{\prime}\right) j(r)=j\left(r^{\prime}\right) j(s)$ which means $j\left(s^{\prime} r\right)=j\left(r^{\prime} s\right)$. That implies $\exists s^{+} \in S$ with $\left.s^{+} s^{\prime} r=s^{+} r^{\prime} s\right)$. Therefore for any other $\left(j^{\prime}, Y^{\prime}\right)$ in $\mathcal{C}_{l, \mathfrak{M} \text { on }}(R, S)$ the subset $j^{\prime}(S)^{-1} j^{\prime}(R)$ is a subring. Moreover we have $s^{+} s^{\prime} \in S$ and $s^{+} r^{\prime}$ satisfy $\left(s^{+} s^{\prime}\right) r=\left(s^{+} r^{\prime}\right) s$. Since they were constructed for an arbitrary $s$ and $r, S$ is left Ore in $R$.

Left reversibility: Let $r, r^{\prime} \in R, s \in S$. Then $r s=r^{\prime} s \Rightarrow j(r) j(s)=$ $j\left(r^{\prime}\right) j(s)$, so by invertibility of $j(s)$ also $j(r)=j\left(r^{\prime}\right)$. But $(j, Y)$ is object in $\mathcal{C}_{l, \mathfrak{M} \text { on }}(R, S)$ so $j(r)=j\left(r^{\prime}\right) \Rightarrow \exists s^{\prime} \in S, s^{\prime} r=s^{\prime} r^{\prime}$.

4.13 Lemma. (i) Let $S$ be a left denominator set. Define the relation $\sim$ on $S \times R$ by

$$
(s, r) \sim\left(s^{\prime}, r^{\prime}\right) \Leftrightarrow(\exists \tilde{s} \in S \exists \tilde{r} \in R)\left(\tilde{s} s^{\prime}=\tilde{r} s \quad \text { and } \tilde{s} r^{\prime}=\tilde{r} r\right) .
$$

Then $\sim$ is an equivalence relation.

(ii) Let $\Sigma$ be a system of left fractions in a category $\mathcal{C}$. For any pair of objects $X, Y$ in $\mathcal{C}$ let $(\Sigma \times \mathcal{C})(X, Y)$ be a class of all diagrams of the form $(X \stackrel{r}{\rightarrow} Z \stackrel{s}{\leftarrow} Y)$ in $\mathcal{C}$. Define a relation $\sim$ on $(\Sigma \times \mathcal{C})(X, Y)$ by

$$
\begin{aligned}
& (X \stackrel{r}{\rightarrow} Z \stackrel{s}{\leftarrow} Y) \sim\left(X \stackrel{r^{\prime}}{\rightarrow} Z^{\prime} \stackrel{s^{\prime}}{\leftarrow} Y\right) \\
& \quad \Leftrightarrow \exists(X \stackrel{\tilde{r}}{\leftarrow} B \stackrel{\tilde{s}}{\rightarrow} Y),\left\{\begin{array}{l}
\tilde{s} \circ s^{\prime}=\tilde{r} \circ s: B \rightarrow Z \\
\tilde{s} \circ r^{\prime}=\tilde{r} \circ r: B \rightarrow Z^{\prime}
\end{array} .\right.
\end{aligned}
$$

The latter condition can be depicted by saying that the diagram

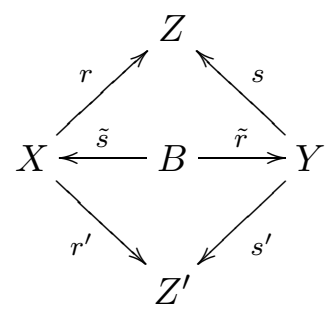

commutes. Conclusion: $\sim$ is an equivalence relation. 
Here $(X \stackrel{\tilde{r}}{\leftarrow} B \stackrel{\tilde{s}}{\rightarrow} Y)$ is not a diagram in $\left(\Sigma^{\mathrm{op}} \times \mathcal{C}\right)(X, Y)$.

Proof. Reflexivity is trivial.

Symmetry: By Ore $\exists r_{1} \in R, s_{1} \in S$ with $r_{1} s=s_{1} s^{\prime}$. Also $\exists r_{2} \in$

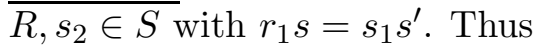

$$
r_{2} \tilde{r} s^{\prime}=r_{2} \tilde{s} s=s_{2} r_{1} s=s_{2} s_{1} s^{\prime} .
$$

In other words $r_{2} \tilde{r}-s_{2} s_{1} \in I_{S}$. Thus by the left reversibility, $\exists t \in S$ with $t\left(r_{2} \tilde{r}-s_{2} s_{1}\right)=0$. Therefore $t\left(r_{2} \tilde{r}-s_{2} s_{1}\right) r^{\prime}=0$, hence $t s_{2} s_{1} r^{\prime}=$ $t r_{2} \tilde{s} r$. Compare with $t s_{2} s_{1} s^{\prime}=t r_{2} \tilde{s} s$ to see that $\left(s^{\prime}, r^{\prime}\right) \sim(s, r)$.

Transitivity: Assume $(s, r) \sim\left(s^{\prime}, r^{\prime}\right)$ and $\left(s^{\prime}, r^{\prime}\right) \sim\left(s^{\prime \prime}, r^{\prime \prime}\right)$. This means

$$
\exists \tilde{s}, \tilde{\tilde{s}} \in S \exists \tilde{r}, \tilde{\tilde{r}} \in R \quad\left\{\begin{array}{lll}
\tilde{s} s=\tilde{r} s^{\prime} & \tilde{\tilde{s}} s^{\prime}=\tilde{\tilde{r}} s^{\prime \prime} \\
\tilde{s} r=\tilde{r} r^{\prime} & \tilde{\tilde{s}} r^{\prime}=\tilde{\tilde{r}} r^{\prime \prime}
\end{array}\right.
$$

$S$ is left Ore, hence $\exists s_{*} \in S \exists r_{*} \in R$ with $s_{*} \tilde{r}=r_{*} \tilde{\tilde{s}}$. Therefore

$$
\begin{aligned}
& \left(s_{*} \tilde{s}\right) s=s_{*} \tilde{r} s^{\prime}=r_{*} \tilde{\tilde{s}} s^{\prime}=\left(r_{*} \tilde{\tilde{s}}\right) s^{\prime \prime} \\
& \left(s_{*} \tilde{s}\right) r=s_{*} \tilde{r} r^{\prime}=r_{*} \tilde{\tilde{s}} r^{\prime}=\left(r_{*} \tilde{\tilde{s}}\right) r^{\prime \prime}
\end{aligned}
$$

Hence $(s, r) \sim\left(s^{\prime \prime}, r^{\prime \prime}\right)$.

4.14 Simplifying observation. Consider a family of arrows $(s, r) \rightarrow\left((p s)^{-1},(p r)\right)$ where $p s \in S$. Then $(s, r) \sim\left((p s)^{-1},(p r)\right)$. If some property $\mathcal{P}$ of elements of $S \times R$ does not change along such arrows, then $\mathcal{P}$ is well-defined on classes $s^{-1} r:=[s, r] / \sim \in S \times R / \sim$.

Proof. Clearly every $\sim$-arrow is a composition of one such arrow and an inverse of another such arrow.

4.15 Lemma. If $t \in S$ and $t r=t r^{\prime}$ (by reversibility even better if $\left.r t=r^{\prime} t\right)$ then $\left(s, r_{1} r r_{2}\right) \sim\left(s, r_{1} r^{\prime} r_{2}\right)$.

Proof. There are $t^{\prime} \in S, r_{1}^{\prime} \in R$ with $r_{1}^{\prime} t=t^{\prime} r_{1}$. Then $\left(s, r_{1} r r_{2}\right) \sim$ $\left(t^{\prime} s, t^{\prime} r_{1} r r_{2}\right) \sim\left(t^{\prime} s, r_{1}^{\prime} t r r_{2}\right) \sim\left(t^{\prime} s, r_{1}^{\prime} t r^{\prime} r_{2}\right) \sim\left(t^{\prime} s, t^{\prime} r_{1} r^{\prime} r_{2}\right) \sim\left(s, r_{1} r^{\prime} r_{2}\right)$.

4.16 Proposition. For $(j, Y)$ in $\mathrm{Ob}_{l, \mathfrak{D}}(R, S)$ the statement

$$
(\forall y \in Y \quad \exists s \in S \quad \exists r \in R)\left(y=j(s)^{-1} j(r)\right)
$$

holds iff $(j, Y)$ is a universal initial object in $\mathcal{C}=\mathcal{C}_{\mathfrak{D}}(R, S)$.

Proof. $\quad(\Leftarrow)$ Let $(j, Y) \in \mathcal{C}_{l}$ be universal in $\mathcal{C}$. Suppose $Y_{0}:=$ $j(S)^{-1} j(R)$ is a proper subring of $Y$. We'll denote by $j_{0}$ the map from $R$ to $Y_{0}$ agreeing with $j$ elementwise. Then $\left(j_{0}, Y_{0}\right)$ is an object in $\mathcal{C}_{l}$ and the inclusion $i: Y_{0} \rightarrow Y$ is a morphism from $\left(j_{0}, Y_{0}\right)$ into $(j, Y)$. By universality of $(j, Y)$ there is a morphism $i^{\prime}:(j, Y) \rightarrow\left(j_{0}, Y_{0}\right)$. The 
composition of morphisms $i \circ i^{\prime}$ is an automorphism of $(j, Y)$ clearly different from the identity, contradicting the universality of $(j, Y)$.

$(\Rightarrow)$ Let $(j, Y)$ satisfy (2) and let $\left(j^{\prime}, Y^{\prime}\right)$ be any object in $\mathcal{C}(R, S)$. We want to prove that there is unique map $i: Y \rightarrow Y^{\prime}$ which satisfies $i(j(r))=j^{\prime}(r) \forall r \in R$. Note that $i\left(j(s)^{-1} j(s)\right)=i\left(j(s)^{-1}\right) j^{\prime}(s)$ implies $i\left(j(s)^{-1}\right)=j^{\prime}(s)^{-1}$. Thus $i\left(j(s)^{-1} j(r)\right)=j^{\prime}(s)^{-1} j^{\prime}(r)$ so that the value of $i$ is forced for all elements in $Y$ proving the uniqueness.

This formula sets $i$ independently of choice of $s$ and $r$. Indeed, if $j(s)^{-1} j(r)=j\left(s^{\prime}\right)^{-1} j\left(r^{\prime}\right)$ then $j(r)=j(s) j\left(s^{\prime}\right)^{-1} j\left(r^{\prime}\right)$. As $j(S)$ is left Ore in $j(R)$, we can find $\tilde{s} \in S$ and $\tilde{r} \in R$ such that $j(\tilde{r}) j\left(s^{\prime}\right)=j(\tilde{s}) j(s)$ and therefore $j(s) j\left(s^{\prime}\right)^{-1}=j(\tilde{s})^{-1} j(\tilde{r})$. Thus $j(r)=j(\tilde{s})^{-1} j(\tilde{r}) j\left(r^{\prime}\right)$ or $j(\tilde{s}) j(r)=j(\tilde{r}) j\left(r^{\prime}\right)$ and, finally, $j(\tilde{s} r)=j\left(\tilde{r} r^{\prime}\right)$. Thus $\exists s^{+} \in S$, $s^{+} \tilde{s} r=s^{+} \tilde{r} r^{\prime}$. Starting here and reversing the chain of arguments, but with $j^{\prime}$ instead of $j$, we get $j^{\prime}(s)^{-1} j^{\prime}(r)=j^{\prime}\left(s^{\prime}\right)^{-1} j^{\prime}\left(r^{\prime}\right)$.

4.17 Theorem. If $S$ is a left denominator set in $R$ then the universal object $(j, Y)$ in $\mathcal{C}_{l, \mathfrak{M} \text { on }}(R, S)$ exists.

Proof. We will construct a universal object $(j, Y) \equiv\left(\iota, S^{-1} R\right)$. As a set, $S^{-1} R:=(S \times R) / \sim$. Let $[s, r]$, and, by abuse of notation, let $s^{-1} r$ also denote the $\sim$-equivalence class of a pair $(s, r) \in S \times R$. Notice that $1^{-1} r=1^{-1} r^{\prime}$ may hold even for some $r \neq r^{\prime}$, namely when $\exists s \in S$ and $r, r^{\prime}$ with $s r=s r^{\prime}$. The equivalence relation is forced by (11).

Multiplication $s_{1}^{-1} r_{1} \cdot s_{2}^{-1} r_{2}:=\left(\tilde{s} s_{1}\right)^{-1}\left(\tilde{r} r_{2}\right)$ where $\tilde{r} \in R, \tilde{s} \in S$ satisfy $\overline{\tilde{r}} s_{2}=\tilde{s} r_{1}\left(\right.$ thus $\tilde{s}^{-1} \tilde{r}=r_{1} s_{2}^{-1}$ ), as in the diagram:

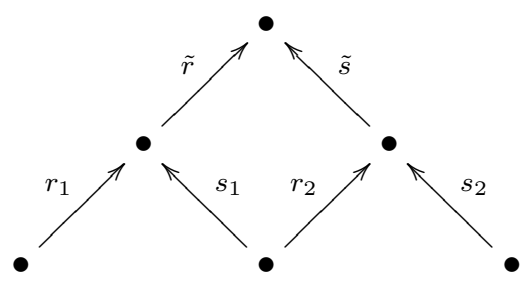

If we choose another pair of multipliers $\tilde{\tilde{r}} \in R, \tilde{\tilde{s}} \in S$ with $\tilde{\tilde{r}} s_{2}=\tilde{\tilde{s}} r_{1}$ instead, then by the left Ore condition we can choose $r_{*} \in R, s_{*} \in S$ with $s_{*} \tilde{s}=r_{*} \tilde{\tilde{s}}$. Then

$$
r_{*} \tilde{\tilde{r}} s_{2}=r_{*} \tilde{\tilde{s}} r_{1}=s_{*} \tilde{s} r_{1}=s_{*} \tilde{r} s_{2}
$$

and therefore $r_{*} \tilde{\tilde{r}}-s_{*} \tilde{r} \in I_{S}$.

In other words, $\exists s^{+} \in S$ with $s^{+} r_{*} \tilde{\tilde{r}}=s^{+} s_{*} \tilde{r}$. 
Therefore we have

$$
\begin{aligned}
& s^{+} s_{*} \tilde{r} r_{2}=s^{+} r_{*} \tilde{\tilde{r}} r_{2} \\
& s^{+} s_{*} \tilde{s} s_{1}=s^{+} r_{*} \tilde{\tilde{s}} s_{1}
\end{aligned}
$$

which proves $\left(\tilde{s} s_{1}\right)^{-1}\left(\tilde{r} r_{2}\right)=\left(\tilde{\tilde{s}} s_{1}\right)^{-1}\left(\tilde{\tilde{r}} r_{2}\right)$. Thus multiplication is well defined as a map $\mu_{1}:(S \times R) \times(S \times R) \rightarrow S^{-1} R$.

We have to show that $\mu_{1}$ factors to $\mu: S^{-1} R \times S^{-1} R \rightarrow S^{-1} R$.

By 4.14, it is sufficient to show that $a=\mu_{1}\left(\left(s_{1}, r_{1}\right),\left(s_{2}, r_{2}\right)\right)$ equals $b=\mu_{1}\left(\left((r s)_{1},\left(r r_{1}\right)\right),\left((p s)_{2},\left(p r_{2}\right)\right)\right)$ whenever $r s \in S$ and $p s \in S$.

$s_{2}^{\prime} r_{1}=r_{1}^{\prime} s_{2}$ for some $s_{2}^{\prime} \in S$ and $r_{1}^{\prime} \in R$. Then $a=\left(s_{2}^{\prime} s_{1}\right)^{-1}\left(r_{1}^{\prime} r_{2}\right)$. As $p s_{2} \in S, \exists p^{\prime} \in R, s_{*} \in S$ with $p^{\prime}\left(p s_{2}\right)=s_{*} r_{1}^{\prime} s_{2}=s_{*} s_{2}^{\prime} r_{1}$. Furthermore, $s_{\sharp} r=p_{\sharp} s_{*} s_{2}^{\prime}$ for some $s_{\sharp} \in S$ and $p_{\sharp} \in R$. Putting these together, we infer $s_{\sharp}\left(r r_{1}\right)=p_{\sharp} s_{*} s_{2}^{\prime} r_{1}=p_{\sharp} p^{\prime} p s_{2}$ and therefore $\left(r r_{1}\right)\left(p s_{2}\right)^{-1} \rightarrow$ $s_{\sharp}^{-1}\left(p_{\sharp} p^{\prime}\right)$, i.e., be definition, that $b=\left(s_{\sharp} r s_{1}\right)^{-1}\left(p_{\sharp} p^{\prime} p r_{2}\right)$, hence by above, $b=\left(p_{\sharp} s_{*} s_{2}^{\prime} s_{1}\right)^{-1}\left(p_{\sharp} p^{\prime} p r_{2}\right)$. Now use lemma 4.15 and $\left(p^{\prime} p\right) s_{2}=$ $\left(s_{*} r_{1}^{\prime}\right) s_{2}$ to conclude $b=\left(p_{\sharp} s_{*} s_{2}^{\prime} s_{1}\right)^{-1}\left(p_{\sharp} s_{*} r_{1}^{\prime} r_{2}\right)=\left(s_{2}^{\prime} s_{1}\right)^{-1}\left(r_{1}^{\prime} r_{2}\right)=a$.

Hence $\mu$ is well-defined. The unit element is clearly $1=1^{-1} 1$. We need to show associativity of $\mu$. The product $s_{1}^{-1} r_{1} \cdot s_{2}^{-1} r_{2} \cdot s_{3}^{-1} r_{3}$ does not depend on the bracketing, essentially because one can complete the following commutative diagram of elements in $R$ (the composition is the multiplication in $R$ : any pair of straight-line (composed) arrows with the same target is identified with a pair in $S \times R)$ :

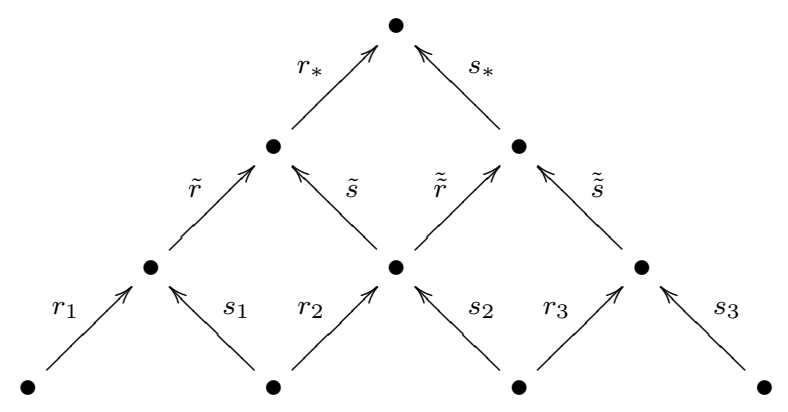

Finally, the construction gives the universal object because it clearly satisfies the equivalent condition in $\mathbf{4 . 1 6}$.

\section{Ore localization for rings}

5.1 Exercise. The two left Ore conditions together immediately imply the combined left Ore condition: 
If $n \in R$ is such that $n s=0$ for some $s \in S$, then for every $r \in R$ there are $s^{\prime} \in R, r^{\prime} \in R$ such that $r^{\prime} n=s^{\prime} r$.

It is sometimes useful to quote this property in order to avoid introducing additional variables needed for deriving it.

5.2 Lemma. Let $f: R \rightarrow R^{\prime}$ be a ring morphism and $S \subset R$ Ore. Then $f(S)$ is an Ore set in $R$.

5.3 Notation. In this section we are concerned only with unital rings. Thus $\mathcal{C}(R, S):=\mathcal{C}_{\text {Rings }}(R, S)$.

5.4 Notation. For any $S \subset R$ let $I_{S}:=\{n \in R \mid \exists s \in S$, sn $=0\}$. $I_{S}$ is clearly a right ideal. If $S$ is a left Ore set, then $s n=0$ and the left Ore condition imply that $\forall r \in R \exists s_{0} \in S, r_{0} \in R$ with $r_{0} s=s_{0} r$, hence $s_{0} r n=r_{0} s n=0$. Thus $I_{S}$ is then a 2-sided ideal.

5.5 Corollary. If $S^{-1} R$ exist then $\forall(j, Y) \in \mathcal{C}(R, S)$, $\operatorname{ker} j \subset I_{S}$. In particular, an Ore localization of a domain is a domain.

5.6 Theorem. If $S$ is a left denominator set in $R$ then the universal object $(j, Y)$ in $\mathcal{C}_{l}(R, S)$ exists.

Proof. In 4.17 we constructed a monoid structure on $Y=S \times$ $R / \sim$. We exhibit an additive structure on $Y$ such that $j$ is a ring map and $(j, Y)$ is indeed universal.

Addition: Suppose we are given two fractions with representatives $\left(s_{1}, r_{1}\right)$ and $\left(s_{2}, r_{2}\right)$. Then by the left Ore condition we find $\tilde{s} \in S$, $\tilde{r} \in R$ such that $\tilde{s} s_{1}=\tilde{r} s_{2}$. The sum is then defined as

$$
s_{1}^{-1} r_{1}+s_{2}^{-1} r_{2}:=\left(\tilde{s} s_{1}\right)^{-1}\left(\tilde{s} r_{1}+\tilde{r} r_{2}\right)
$$

Suppose we have chosen $\left(\tilde{\tilde{s}}, \tilde{\tilde{r}}^{\prime}\right) \in S \times R$ with $\tilde{\tilde{s}} s_{1}=\tilde{\tilde{r}} s_{2}$, instead of $(\tilde{s}, \tilde{r})$. Then by left Ore we find $s_{*} \in S$ and $r_{*} \in R$ such that $s_{*} \tilde{s}=r_{*} \tilde{\tilde{s}}$. Then

$$
r_{*} \tilde{\tilde{r}} s_{2}=r_{*} \tilde{\tilde{s}} s_{1}=s_{*} \tilde{s} s_{1}=s_{*} \tilde{r} s_{2}
$$

hence $\left(s_{*} \tilde{r}-r_{*} \tilde{\tilde{s}}\right) \in I_{S}$, i.e. $\exists s^{\sharp} \in S$ with

$$
s^{\sharp} s_{*} \tilde{r}=s^{\sharp} r_{*} \tilde{\tilde{s}}
$$

Then

$$
\begin{aligned}
& \left(s^{\sharp} s_{*}\right)\left(\tilde{s} r_{1}+\tilde{r} r_{2}\right)=\left(s^{\sharp} r_{*}\right)\left(\tilde{s} r_{1}+\tilde{\tilde{s}} r_{2}\right) \\
& \left(s^{\sharp} s_{*}\right)\left(\tilde{s} s_{1}\right)=\left(s^{\sharp} r_{*}\right)\left(\tilde{\tilde{s}} s_{2}\right)
\end{aligned}
$$

Conclusion: $\left(\tilde{s} s, \tilde{s} r_{1}+\tilde{r} r_{2}\right) \sim\left(\tilde{\tilde{s}} s, \tilde{\tilde{s}} r_{1}+\tilde{\tilde{r}} r_{2}\right)$, as required.

Now let's check that the sum does not depend on the choice of the representative of the first summand. Suppose we are given two 
representatives of the first fraction $s_{1}^{-1} r_{1}=s_{1}^{\prime-1} r_{1}^{\prime}$. Then for some $\left(s^{*}, r^{*}\right) \in S \times R$ we have

$$
s_{*} s_{1}=r_{*} s_{1}^{\prime} \quad \text { and } \quad s_{*} r_{1}=r_{*} r_{1}^{\prime}
$$

Second fraction in $s_{2}^{-1} r_{2}$. Choose

$$
(\tilde{\tilde{s}}, \tilde{\tilde{r}}) \in S \times R \quad \text { with } \quad \tilde{\tilde{s}} s_{1}^{\prime}=\tilde{\tilde{r}} s_{2} .
$$

Now choose $\left(s_{\sharp}, r_{\sharp}\right) \in S \times R$ such that $s_{\sharp} r_{*}=r_{\sharp} s_{0}$. Then $\left(r_{\sharp} \tilde{\tilde{r}}\right) s_{2}=$ $r_{\sharp} \tilde{\tilde{s}} s_{1}^{\prime}=s_{\sharp} r_{*} s_{1}^{\prime}=\left(s_{\sharp} s_{*}\right) s_{1}$ and $\left(r_{\sharp} \tilde{\tilde{s}}\right) r_{1}^{\prime}=s_{\sharp} r_{*} r_{1}^{\prime}=\left(s_{\sharp} s_{*}\right) r_{1}$. Therefore

$$
\begin{aligned}
s_{1}^{-1} r_{1}+s_{2}^{-1} r_{2} & =\left(s_{\sharp} s_{*} s_{1}\right)^{-1}\left(s_{\sharp} s_{*} r_{1}+r_{\sharp} \tilde{\tilde{r}} r_{2}\right) \\
& =\left(r_{\sharp} \tilde{\tilde{s}} s_{1}^{\prime}\right)^{-1}\left(r_{\sharp} \tilde{\tilde{s}} r_{1}^{\prime}+r_{\sharp} \tilde{\tilde{r}} r_{2}\right) \\
& =\left(\tilde{\tilde{s}} s_{1}^{\prime}\right)^{-1}\left(\tilde{\tilde{s}} r_{1}^{\prime}+\tilde{\tilde{r}} r_{2}\right) \\
& =s_{1}^{\prime-1} r_{1}^{\prime}+s_{2}^{-1} r_{2}
\end{aligned}
$$

We should also check that the sum does not depend on the second summand. This proof would not be symmetric to this one as our definition of the sum is not. We shall choose an indirect proof. Denote the set-theoretic quotient map by $p: S \times R \rightarrow S^{-1} R$. By now we have completed the proof that addition as a map from

$$
\tilde{+}: S^{-1} R \times(S \times R) \rightarrow S^{-1} R
$$

is well defined. Now we prove that the map

$$
\tilde{+}(p \times \mathrm{id}) \tau:(S \times R) \times(S \times R) \rightarrow S^{-1} R
$$

where $\tau$ is the transposition of factors coincides with $\tilde{+}(p \times$ id $)$. Thus we have a well-defined addition as a map defined on $S^{-1} R \times S^{-1} R$ which is then automatically commutative. It is sufficient to prove that for any two pairs $\left(s_{1}, r_{1}\right)$ and $\left(s_{2}, r_{2}\right)$ and any

$$
\tilde{s}, \tilde{\tilde{s}} \in S, \tilde{r}, \tilde{\tilde{r}} \in R \text { with } \quad \tilde{s} s_{1}=\tilde{r} s_{2}, \quad \tilde{\tilde{r}} s_{1}=\tilde{\tilde{s}} s_{2}
$$

the classes

$$
\begin{aligned}
& \left(\tilde{s} s_{1}\right)^{-1}\left(\tilde{\tilde{s}} r_{1}+\tilde{r} r_{2}\right) \\
& \left(\tilde{\tilde{s}} s_{2}\right)^{-1}\left(\tilde{\tilde{r}} r_{1}+\tilde{\tilde{s}} r_{2}\right)
\end{aligned}
$$

coincide in $S^{-1} R$. For that purpose, choose $s_{\sharp} \in S$ and $r_{\sharp} \in R$ such that $s_{\sharp} \tilde{r}=r_{\sharp} \tilde{\tilde{s}}$. Then

$$
r_{\sharp} \tilde{s} s_{1}=s_{\sharp} \tilde{r} s_{2}=r_{\sharp} \tilde{\tilde{s}} s_{2} .
$$


Next $r_{\sharp} \tilde{\tilde{r}} s_{1}=r_{\sharp} \tilde{\tilde{s}} s_{2}=s_{\sharp} \tilde{r} s_{2}=s_{\sharp} \tilde{s} s_{1}$, and therefore $\left(r_{\sharp} \tilde{\tilde{r}}-s_{\sharp} \tilde{s}\right) \in I_{S}$. Thus $\exists s^{+} \in S$ with

$$
s^{+} r_{\sharp} \tilde{\tilde{r}}-s^{+} s_{\sharp} \tilde{s}=0 .
$$

In particular, $s^{+} r_{\sharp} \tilde{\tilde{r}} r_{1}=s^{+} s_{\sharp} \tilde{s} r_{1}=0$. Thus

$$
\begin{aligned}
\left(\tilde{s} s_{1}\right)^{-1}\left(\tilde{s} r_{1}+\tilde{r} r_{2}\right) & =\left(s^{+} s_{\sharp} \tilde{s} s_{1}\right)^{-1}\left(s^{+} s_{\sharp} \tilde{s} r_{1}+s^{+} s_{\sharp} \tilde{r} r_{2}\right) \\
& =\left(s^{+} r_{\sharp} \tilde{\tilde{s}} s_{1}\right)^{-1}\left(s^{+} r_{\sharp} \tilde{\tilde{r}} r_{1}+s^{+} r_{\sharp} \tilde{\tilde{s}} r_{2}\right) \\
& =\left(\tilde{\tilde{s}} s_{1}\right)^{-1}\left(\tilde{\tilde{r}} r_{1}+\tilde{\tilde{s}} r_{2}\right)
\end{aligned}
$$

The associativity of addition is left to the reader.

The distributivity law follows by $\mathbf{4 . 8}$.

The element $1^{-1} 0$ in $S^{-1} R$ is the zero and thus $S^{-1} R$ is equipped with a natural unital ring structure.

Define $\iota: R \rightarrow S^{-1} R$ by $\iota(r)=[1, r]=1^{-1} r$. Check that $\iota$ is a unital ring homomorphism. Check that $\iota(S)$ consists of units and that $\iota(S)^{-1} \iota(R)=Y$. Pair $\left(\iota, S^{-1} R\right)$ is a universal object in $\mathcal{C}_{l}(R, S)$, as it clearly satisfies the equivalent condition in $\mathbf{4 . 1 6}$.

5.7 Right Ore conditions, and right Ore localizations with respect to $S \subset R$, are by definition the left Ore conditions and localizations with respect to $S \subset R^{\mathrm{op}}$. The right ring of fractions is denoted $R S^{-1}$ : $\left(S^{-1} R^{\mathrm{op}}\right)^{\mathrm{op}}$. It consists of certain equivalence pairs $r s^{-1}:=[(r, s)]$, where $(r, s) \in R \times S$.

\section{Practical criteria for Ore sets}

6.1 Theorem. (i) Let $S$ and $S^{\prime}$ be multiplicative sets in ring $R$, where $S$ is also left Ore in $R$. If

1. for a map $j: R \rightarrow Y$ of unital rings, the image $j(S)$ consists of units in $Y$ iff the image $j\left(S^{\prime}\right)$ consists of units in $Y$;

2. $s r=0$ for some $s \in S$ iff $\exists s^{\prime} \in S^{\prime}$ with $s^{\prime} r=0$.

Then $S^{\prime}$ is left Ore as well and $S^{-1} R$ is canonically isomorphic to $S^{\prime-1} R$.

Proof. Under the above conditions the categories $\mathcal{C}_{l}(R, S)$ and $\mathcal{C}_{l}\left(R, S^{\prime}\right)$ are identical, so call them simply $\mathcal{C}$. The left Ore condition is equivalent to the existence of an initial object in $\mathcal{C}$; and the 2 localizations are just the 2 choices of an initial object, hence there is a unique isomorphism in $\mathcal{C}$ between them; its image under the forgetful functor $\mathcal{C} \rightarrow R$-Mod into the category of unital rings, is the canonical isomorphism as required. 
6.2 The left Ore condition is often checked inductively on a filtration, or ordered set of generators. To disseminate this kind of reasoning we will temporarily use some nonstandard notation which generalizes the left Ore condition. One fixes a (only) multiplicative set $S \subset R$. For any subset $A \subset R$, and any $(s, r) \in S \times A \subset S \times R$, introduce predicate

$$
\operatorname{lOre}(s, r \uparrow A):=\operatorname{lOre}_{S, R}(s, r \uparrow A) \equiv\left(\exists s^{\prime} \in S, \exists r^{\prime} \in A, s^{\prime} r=r^{\prime} s\right),
$$

where $S, R$ in subscripts may be skipped if known from context. Moreover if $A=R$ then $\uparrow A$ may be skipped from the notation. For example, $\operatorname{lOre}(s, r)=\operatorname{lOre}_{S, R}(s, r \uparrow R)$.

For any subsets $A_{0} \subset A$ and $S_{0} \subset S$, abbreviate

$$
\operatorname{lOre}\left(S_{0}, A_{0} \uparrow A\right) \equiv\left(\forall s \in S_{0}, \forall r \in R_{0}, \operatorname{lOre}_{S, R}(s, r \uparrow A)\right),
$$

with rules for skipping $\uparrow A$ as before. For example, $\operatorname{lOre}(S, R)$ is simply the left Ore condition for $S \subset R$.

Finally,

$$
\operatorname{slOre}\left(S_{0}, A\right) \equiv \operatorname{lOre}_{S, R}\left(S_{0}, A \uparrow A\right) .
$$

For an additive subgroup $A \subset R$ consider also the relative versions, e.g.

$$
\text { rel }-\operatorname{lOre}_{S, R}(s, r ; I) \equiv\left(\exists s^{\prime} \in S, \exists r^{\prime} \in R, s^{\prime} r-r^{\prime} s \in A\right) .
$$

If $A=I$ is an ideal, then this predicate is suitable for study of some (non-Ore) generalizations.

6.3 Extending Ore property Let $A, B \subset R$ be additive subgroups of $R, A \subset B \subset R$, and $S \subset R$ multiplicative subset.

(i) $\quad(\operatorname{lOre}(S, A)$ and $\operatorname{rel}-\operatorname{lOre}(S, R ; A)) \Rightarrow \operatorname{lOre}(S, R)$

(ii) $\quad(\operatorname{lOre}(S, A \uparrow B)$ and $\operatorname{rel}-\operatorname{slOre}(S, B ; A)) \Rightarrow \operatorname{lOre}(S, B)$

(iii) $\quad(\operatorname{lOre}(S, A \uparrow B)$ and $\operatorname{rel}-\operatorname{slOre}(S, B ; A)$

$$
\text { and } S B \subset B) \Rightarrow \operatorname{slOre}(S, B)
$$

Proof. (i) is clearly the $B=R$ case of (ii). Let $b \in B$ and $s \in S$. Then rel - $\operatorname{slOre}(S, B ; A))$ means that $\exists s^{\prime} \in S, \exists b^{\prime} \in B, \exists a \in A$ such that $s^{\prime} b-b^{\prime} s=a$. Now we compare $s$ and $a$. There are $b_{1} \in B, s_{1} \in S$ such that $b_{1} s=s_{1} a$. Thus $s_{1} s^{\prime} b-s_{1} b^{\prime} s=s_{1} a=b_{1} s$, and finally, $\left(s_{1} s^{\prime}\right) b=\left(s_{1} b^{\prime}+b_{1}\right) s . S$ is multiplicative hence (ii), and if $S B \subset R$ then $s_{1} b^{\prime}+b_{1} \in B$ hence (iii). 
6.3a Remark. The above condition is usually checked for generators only. Also we can iterate the above criterion if we have a finite or denumerable family of nested subrings, for which the induction is convenient. One may also need to nest subsets of $S$, with refined criteria, like $\operatorname{lore}\left(S_{1} \uparrow S_{2}, A_{1} \uparrow A_{2}\right)$, where the $\uparrow S_{2}$ means that $s^{\prime}$ may be chosen in $S_{2}$.

6.4 Lemma. If $S_{1}$ multiplicatively generates $S$, and $A \subset R$ then

$$
\begin{gathered}
\operatorname{lOre}\left(S_{1}, R\right) \Leftrightarrow \operatorname{lOre}(S, R), \\
\operatorname{slOre}\left(S_{1}, A\right) \Leftrightarrow \operatorname{slOre}(S, A) .
\end{gathered}
$$

Proof. The first statement is clearly a particular case of the second. Hence we prove the second statement; the nontrivial direction is $\Rightarrow$. By assumption, the set $S$ can be written as a nested union $\cup_{n \geq 0} S_{n}$ where $S_{n}$ consists of all those $s \in S$ which can be expressed as a product $\prod_{k=1}^{n^{\prime}} s_{k}$ with $n^{\prime} \leq n$ and $s_{k} \in S_{k}$; (hence $S_{1}$ is as the same as before). The assumption is $\operatorname{slOre}\left(S_{1}, A\right)$, hence by induction it is enough to prove that $\operatorname{slOre}\left(S_{n}, A\right) \Rightarrow \operatorname{slOre}\left(S_{n+1}, A\right)$ for all $n \geq 1$. Take $s=s_{1} s_{2} \cdots s_{n}$. Then $\operatorname{slOre}\left(S_{n}, A\right)$ means that for any $a \in A$ we have

$$
\begin{aligned}
& \exists a^{\prime} \in A \exists s^{\prime} \in S\left(a^{\prime} s_{2} \cdots s_{n}=s^{\prime} a\right), \\
& \exists a^{\prime \prime} \in A \exists s^{\prime \prime} \in S\left(a^{\prime \prime} s_{1}=s^{\prime \prime} a^{\prime}\right)
\end{aligned}
$$

and consequently

$$
a^{\prime \prime} s_{1} s_{2} \ldots s_{n}=s^{\prime \prime} a^{\prime} s_{2} \ldots s_{n}=\left(s^{\prime \prime} s^{\prime}\right) a,
$$

giving the conclusion by the multiplicative closedness of $S$.

6.5 Lemma. If $A_{0}^{+}, A^{+} \subset R$ be the additive closure of $A_{0}, A$ respectively, then (obviously)

$$
\operatorname{lOre}\left(S, A_{0} \uparrow A\right) \Rightarrow \operatorname{lOre}\left(S, A_{0}^{+} \uparrow A^{+}\right) .
$$

6.6 Lemma. If $A$ generates $R$ as a ring, then

$$
\operatorname{lOre}(S, A) \Rightarrow \operatorname{lOre}(S, R) \text {. }
$$

Proof. By 6.5 it is enough to check this multiplicativity:

$$
\left(\forall i, \operatorname{lOre}\left(S, c_{i}\right)\right) \Rightarrow \operatorname{lOre}\left(S, c_{n} \cdots c_{1}\right),
$$

when $c_{i} \in A$. However, this general statement holds for any choice of $c_{i}$ whatsoever. Namely, if we do not require $c_{i} \in A$, we see, by 
induction on $n$, that it is enough to prove this statement for $n=2$. For $s \in S$ and $c_{1}, c_{2} \in R$ we can find $r_{1}^{\prime}, r_{2}^{\prime} \in R$ and $s^{\prime}, s^{\prime \prime} \in S$, so that $r_{1}^{\prime} s=s^{\prime} c_{1}$ and $r_{2}^{\prime} s^{\prime}=s^{\prime \prime} c_{2}$. The result is

$$
r_{2}^{\prime} r_{1}^{\prime} s=r_{2}^{\prime} s^{\prime} c_{1}=s^{\prime \prime} c_{2} c_{1},
$$

hence the lemma is proved.

6.7 Theorem. If $A \subset B$ and the subring $\bar{A} \subset R$ is also contained in $B$, then for any $S_{1}$ multiplicatively generating a multiplicatively closed set $S$ we have

$$
\begin{gathered}
\operatorname{slOre}\left(S_{1}, A\right) \Rightarrow \operatorname{lOre}(S, \bar{A} \uparrow B), \\
\operatorname{lOre}(S, A) \Rightarrow \operatorname{lOre}(S, \bar{A}) .
\end{gathered}
$$

Proof. We know $\operatorname{slOre}\left(S_{1}, A\right) \Rightarrow \operatorname{slOre}(S, A)$. Hence the first assertion follows from the second by $\bar{A} \subset B$. We proved the second statement in the case $\bar{A}=R$. If $S \subset \bar{A}$, the statement clearly does not say anything more than it would say after replacing $R$ by its subring $\bar{A}$. The proof of the general case is exactly the same, as $s \in R$ is never used, and our calculations and quantifiers may be taken over a bigger overring.

6.8 Warning-theorem. If $A$ generates $R$ as a ring and $S_{1}$ generates $S$ multiplicatively, then it is NOT necessarily true that

$$
\operatorname{lOre}\left(S_{1}, A\right) \Rightarrow \operatorname{lOre}(S, R),
$$

even if $S_{1}$ has only one multiplicative generator. We know from 6.4 that (3) holds if we replace 1Ore by the stronger condition slOre. Nevertheless, various intermediate conditions, standing between lOre and slOre, often utilizing filtrations and combined arguments, are widely used in practice. However it is also common to use (3) without proper justification.

6.8a Counterexample. (proving the warning statement above)

Let $R$ be the ring generated by 4 generators $z_{1}, z_{2}, z_{3}, D$ modulo the following relations:

$$
\begin{aligned}
& D z_{1}=z_{2} z_{3} D, \\
& D^{2} z_{2}=z_{3} z_{1} D, \\
& D^{3} z_{3}=z_{1} z_{2} D .
\end{aligned}
$$

Clearly the (4) are simply the identities needed to check $\operatorname{lOre}\left(S_{1}, A\right)$ where $S_{1}=\{D\}$, and $A=\left\{z_{1}, z_{2}, z_{3}\right\}$. The powers of $D$ on the 
right-hand side which are $1,2,3$ respectively can be $1, p, q$ with the same proof as below, with any integers $p>0$ and $q>1$, but the last inequality is indeed essential (if $p=q=1$, this is not a counterexample at all).

We claim that for any nonnegative integer $n, D^{n} z_{1}=P D^{2}$ does not have a solution for $P \in R$, hence the left Ore condition is not satisfied for the multiplicative set $S=\left\{1, D, D^{2}, \ldots\right\}$. The proof of the claim will be by contradiction, but we need first to study a convenient basis of ring $R$.

A basis of $R$ consists of all ordered monomials where, the righthand side of any of the equations (4) does not appear as a factor. This is obtained using Bergman's diamond lemma [15] with the reduction system $K$ having 3 reductions corresponding to the relations (44) with production arrows from right to left. This reduction system has clearly no ambiguities whatsoever and all reductions send monomials into monomials in generators $z_{1}, z_{2}, z_{3}, D$. It is direct to see using this basis that $D$ is not a zero divisor.

If $S$ is left Ore then there exist $n$ such that

$$
D^{n} z_{1}=P D^{2}, \quad \text { for some } P \in R \text {. }
$$

We suppose that $n \geq 3$, and leave the remaining case to the reader. Equation (15) implies $D^{n-1} z_{2} z_{3} D=P D^{2}$, and as $D$ is not a divisor that $D^{n-1} z_{2} z_{3}=P D$. One writes $P$ as a sum of linearly independent $K$-reduced monomials $P_{i}$. As $D$ is not a zero divisor, monomials $P_{i} D$ are also linearly independent. As all the reductions in $K$ send monomials to monomials, and $D^{n-1} z_{2} z_{3}$ is a $K$-reduced monomial, $D^{n-1} z_{2} z_{3}$ can not be obtained as a sum of more than one of the linearly independent monomials $P_{i} D$, hence $P$ has to be a monomial. The only way that $P D$ in $K$-reduced form (which is $D^{n-1} z_{2} z_{3}$ ) has $z_{3}$ as the most right-hand side factor is that $P=P^{\prime} z_{1} z_{2}$ for some $P^{\prime}$ in $K$-reduced form. Hence we obtain $D^{n-1} z_{2} z_{3}=P^{\prime} z_{1} z_{2} D=P^{\prime} D z_{3}$. Again using basis one can check directly that $Q z_{3}=0$ implies $Q=0$, hence $D^{n-1} z_{2}=P^{\prime} D$. Now $D^{n-3} D^{2} z_{2}=D^{n-3} z_{3} z_{1} D=P^{\prime} D$ implies $D^{n-3} z_{3} z_{1}=P^{\prime}$. This substituted back in the expression for $P$ and the equation (5) gives

$$
D^{n} z_{1}=P^{\prime} z_{1} z_{2} D=D^{n-3} z_{3} z_{1} z_{1} z_{2} D^{2}=D^{n-1} z_{2} D^{2} z_{3} D .
$$

This is a contradiction as the two sides differ even though they are $K$-reduced. 
6.9 Proposition. Let $S$ and $T$ be left Ore sets in some ring $R$. Then the set of all elements of the form st where $s \in S$ and $t \in T$ satisfies the left Ore condition in $R$ (but it is not necessarily multiplicatively closed).

Proof. Suppose the contrary, i.e. there is $s t \in S T$ and $r \in R$, such that we can not find $s^{\prime} \in S, t^{\prime} \in T$ and $r^{\prime} \in B$, so that $r^{\prime} s t=s^{\prime} t^{\prime} r$. Set $T$ is Ore so there are $t^{\prime} \in T$ and $r_{1} \in R$ with $r_{1} t=t^{\prime} r$. Next we can find $s^{\prime} \in S$ and $r_{2} \in R$ so that $r_{2} s=s^{\prime} r_{1}$. Combining, we obtain

$$
s^{\prime} t^{\prime} r=s^{\prime} r_{1} t=r_{2} s t,
$$

with contradiction.

6.10 Proposition. Let $S$ be a left Ore set in a $k$-algebra $R$. The set $1 \otimes S$ of all elements of $R \otimes_{k} R$ of the form $1 \otimes s$ where $s \in S$ is an Ore set.

Proof. $1 \otimes S$ is obviously multiplicatively closed.

If the Ore condition is not true, there is an element $y=\sum_{i=1}^{n} a_{i} \otimes b_{i}$ and an element $s \in S$ such that $(1 \otimes S) y \cap(R \otimes R)(1 \otimes s)=\emptyset$.

We use induction by $n$ to find an element in the intersection. If $n=1$ we simply use that $S$ is left Ore to find $r^{\prime} \in R$ and $s^{\prime} \in S$ such that $r^{\prime} s=s^{\prime} b_{1}$ and we have

$$
\left(1 \otimes s^{\prime}\right)\left(a_{1} \otimes b_{1}\right)=\left(a_{1} \otimes r^{\prime}\right)(1 \otimes s),
$$

which proves the basis of induction.

Suppose we found $s_{j}^{\prime} \in S$ and $z=\sum_{i=1}^{j} a_{i} \otimes r_{i}^{\prime j}$ so that

$$
\left(1 \otimes s_{j}^{\prime}\right)\left(\sum_{i=1}^{j} a_{i} \otimes b_{i}\right)=\left(\sum_{i=1}^{j} a_{i} \otimes r_{i}^{\prime}\right)(1 \otimes s) .
$$

Now we use again the property that $S$ is left Ore to find $r_{j+1}^{j+1} \in R$ and $\bar{s}_{j+1} \in S$ such that

$$
r_{j+1}^{\prime j+1} s=\bar{s}_{j+1} s_{j}^{\prime} b_{j+1} .
$$

$S$ is a multiplicatively closed set so $s_{j+1}^{\prime}=\bar{s}_{j+1} s_{j}^{\prime}$ is an element of $S$. Now we have

$$
\begin{gathered}
\left(1 \otimes s_{j+1}^{\prime}\right)\left(\sum_{i=1}^{j+1} a_{i} \otimes b_{i}\right)=\begin{array}{r}
\left(1 \otimes \bar{s}_{j+1}\right)\left(1 \otimes s_{j}^{\prime}\right)\left(\sum_{i=1}^{j} a_{i} \otimes b_{i}\right)+ \\
+\left(1 \otimes \bar{s}_{j+1}\right)\left(1 \otimes s_{j}^{\prime}\right)\left(a_{j+1} \otimes b_{j+1}\right)
\end{array} \\
=\left(\sum_{i=1}^{j} a_{i} \otimes \bar{s}_{j+1} r_{i}^{\prime j}\right)(1 \otimes s)+\left(a_{j+1} \otimes r_{j+1}^{\prime j+1}\right)(1 \otimes s) \\
=\left(\sum_{i=1}^{j+1} a_{i} \otimes r_{i}^{\prime j+1}\right)(1 \otimes s)
\end{gathered}
$$

where we denoted $r_{i}^{\prime j+1}=\bar{s}_{j+1} r_{i}^{\prime j}$ for $i<j+1$ and $r_{j+1}^{\prime j+1}$ has already been defined. 


\section{Ore localization for modules}

The modern point of view on Ore localization is to express it as a localization functor on some category of modules. The localization map $\iota: R \rightarrow S^{-1} R$ will be replaced by a localization functor $Q_{S}^{*}$ from $R$-Mod to $S^{-1} R$-Mod.

7.1 Let $S$ be a left Ore set in a ring $R$, and $M$ a left $R$-module. Notice that $S^{-1} R$ may be viewed as an $S^{-1} R$ - $R$-bimodule. The module of fractions $S^{-1} M$ of $M$ with respect to Ore set $S$ is the left $S^{-1} R$-module

$$
Q_{S}^{*}(M)=S^{-1} M:=S^{-1} R \otimes_{R} M .
$$

For each morphism $f: M \rightarrow N$, set $Q_{S}^{*}(f):=1 \otimes f: S^{-1} R \otimes_{R} M \rightarrow$ $S^{-1} R \otimes_{R} N$. This defines a localization functor $Q_{S}^{*}: R-\bmod \rightarrow$ $S^{-1} R-\bmod$ whose right adjoint is the obvious forgetful functor $Q_{S *}$ : $S^{-1} R-\bmod \rightarrow R-\bmod$. If $\iota=\iota_{R}: R \rightarrow S^{-1} R$ is the localization map, then define the map of $R$-modules $\iota_{M}: M \rightarrow S^{-1} M$ via $\iota_{M}=\iota_{R} \otimes_{R}$ id i.e. $m \mapsto 1 \otimes m$, also called the localization map. Maps $\iota_{M}$ make together a natural transformation of functors, namely the adjunction $\iota:$ Id $\rightarrow Q_{S *} Q_{S}^{*}$.

7.1a Remark. If $S$ is a right Ore set, and $M$ a left $R$-module, then $M\left[S^{-1}\right]:=R\left[S^{-1}\right] \otimes_{R} M$. If $N$ is a right $R$-module then view $R S^{-1}$ (or $S^{-1} R$ ) as a $R-R S^{-1}$ - (resp. $R-S^{-1} R$ )- bimodule and define $Q_{S}^{*}(N):=N \otimes_{R} R\left[S^{-1}\right]$ (resp. $N \otimes_{R} S^{-1} R$ ). We emphasize that the choice of right vs. left Ore sets is not correlated with the choice of right or left $R$-module categories, at least in the principle of the construction.

7.2 Universal property. For given $R, M, S$ as above we define the category $\mathcal{M}=\mathcal{M}(R, M, S)$. The objects of $\mathcal{M}$ are pairs $(N, h)$ where $N$ is a left $S^{-1} R$-module and $h: M \rightarrow{ }_{R} N$ a map of left $R$-modules. A morphism of pairs $\mu:(N, h) \rightarrow\left(N^{\prime}, h^{\prime}\right)$ is a map $\mu: N \rightarrow N^{\prime}$ of $S^{-1} R$-modules such that $h^{\prime}=\mu \circ h$.

Proposition. The pair $\left(S^{-1} M, \iota_{M}\right)$ is a initial object in $\mathcal{M}$.

Proof. For any pair $(N, h) \in \mathrm{Ob}(\mathcal{M})$ there is a well-defined morphism

$$
\alpha:\left(S^{-1} M, \iota_{M}\right) \rightarrow(N, h) \text { by } \alpha\left(s^{-1} \otimes_{R} m\right)=s^{-1} h(m) .
$$

Let now $\alpha^{\prime}$ be any morphism from $\left(S^{-1} M, \iota\right)$ to $(N, h)$. As $h=\alpha^{\prime} \circ i$ we have

$$
\alpha^{\prime}(1 \otimes m)=h(m)=\alpha(1 \otimes m), \forall m \in M
$$


The elements of the form $1 \otimes m$ generate $S^{-1} M$ as a module over $S^{-1} R$ and therefore $\alpha=\alpha^{\prime}$.

7.3 Elements in the tensor product (6) are of the form $\sum_{i} s_{i}^{-1} \otimes m_{i}$ but such can be added up to a single term of that form, as the fractions can be always put to the common denominator. Namely, by the left Ore condition $\forall s, s^{\prime} \in S \exists \tilde{s} \in S \exists \tilde{r} \in R, s^{\prime} s^{-1}=\tilde{s}^{-1} \tilde{r}$, and therefore

$$
\begin{aligned}
s^{-1} \otimes m+s^{-1} \otimes m^{\prime} & =s^{-1} s^{\prime} s^{-1} \otimes m+s^{-1} \tilde{s}^{-1} \tilde{s} \otimes m^{\prime} \\
& =s^{\prime-1} \tilde{s}^{-1} \otimes\left(\tilde{r} m+\tilde{s} m^{\prime}\right) .
\end{aligned}
$$

Thus every element in $S^{-1} M$ may be written in the form $s^{-1} \otimes_{R} m$, hence there is a surjection of sets $\nu: S \times M \rightarrow S^{-1} M$. The set $S \times M$ may be viewed as a set retract $S \times\{1\} \times M$ of $S \times R \times M$ via the retraction $(s, r, m) \mapsto(s, r m)$. Clearly $\nu$ extends to $\nu^{\prime}: S \times R \times M \rightarrow$ $S^{-1} M$. By the universality of the free abelian group $\mathbb{Z}(S \times R \times M)$ with basis $S \times R \times M, \exists ! \tilde{\nu}^{\prime}: \mathbb{Z}(S \times R \times M) \rightarrow S^{-1} M$ which is additive and extends $\nu^{\prime}$. It is clear by checking on the basis elements $(s, r, m)$ and uniqueness that the composition of the canonical projections $\mathbb{Z}(S \times$ $R \times M) \rightarrow \mathbb{Z}\left(S^{-1} R \times M\right) \rightarrow S^{-1} R \otimes_{R} M$ equals $\tilde{\nu^{\prime}}$.

For $r \in R$ for which $r s \in S, s^{-1} \otimes_{R} m=(r s)^{-1} r \otimes_{R} m=(r s)^{-1} \otimes_{R}$ $r m$ implying that $\operatorname{ker} \nu^{\prime} \subset \mathbb{Z}(S \times M)$ contains all differences $(s, m)-$ $\left(s^{\prime}, m^{\prime}\right)$ in $\mathbb{Z}(S \times M)$ of pairs in $S \times M$ which are equivalent via

$$
(s, m) \sim\left(s^{\prime}, m^{\prime}\right) \Leftrightarrow \exists r, r^{\prime} \in R \quad r s=r^{\prime} s^{\prime} \in S \text { and } r m=r^{\prime} m^{\prime} .
$$

Lemma. (i) On $(S \times M) / \sim$ there is a unique binary operation + such that

$$
(s, m)+\left(s, m^{\prime}\right) \sim\left(s, m+m^{\prime}\right) .
$$

(ii) $((S \times M) / \sim,+)$ is an abelian group. Hence by the universality of the free abelian group, the composition $S \times R \times M \rightarrow S \times M \rightarrow$ $(S \times M) / \sim$ extends to a unique map $p: \mathbb{Z}(S \times R \times M) \rightarrow(S \times M) / \sim$ of abelian groups.

(iii) The map $p$ factors to a map $p^{\prime}: S^{-1} R \otimes_{R} M \rightarrow(S \times M) / \sim$.

(iv) $p^{\prime}$ is an inverse of $\nu$, hence $p^{\prime}$ respects addition.

Proof. (i) Uniqueness. Suppose there are two such operations, $+_{1},+_{2}$ and two classes $\left(s_{1}, m_{1}\right)$ and $\left(s_{2}, m_{2}\right)$ on which $+_{1}$ and $+_{2}$ disagree. By the left Ore condition choose $\tilde{s} \in S, \tilde{r} \in R$ with $\tilde{s} s_{1}=\tilde{r} s_{2}$. Then $\left(s_{1}, m_{1}\right)+_{i}\left(s_{2}, m_{2}\right) \sim\left(\tilde{s} s_{1}, \tilde{s} m_{1}\right)+_{i}\left(\tilde{r} s_{2}, \tilde{r} m_{2}\right) \sim\left(\tilde{s} s_{1}, \tilde{s} m_{1}+\tilde{r} m_{2}\right)$ which agree for $i=1,2$, giving a contradiction.

Existence. Take $\left(s_{1}, m_{1}\right)+\left(s_{2}, m_{2}\right):=\left(\tilde{r}_{1} s_{1}, \tilde{r}_{1} m_{1}+\tilde{r}_{2} m_{2}\right)$ with any choice of $\tilde{r}_{1}, \tilde{r}_{2}$ such that $\tilde{r}_{1} s_{1}=\tilde{r}_{2} s_{2} \in S$. We verify that the class 
of the result does not depend on the choices. If $s_{1}, m_{1}$ are replaced by $r s_{1} \in S, r m_{1} \in M$ we can by the combined Ore condition choose $r_{*}, s_{*}$ with $s_{*} r=r_{*} \tilde{r}_{1}$, hence $S \ni s_{*} r s_{1}=r_{*} \tilde{r}_{1} s_{1}=r_{*} \tilde{r}_{2} s_{2}$. Hence the rule for the sum gives $\left(s_{*} r s_{1}, s_{*} r m_{1}+r_{*} \tilde{r}_{2} m_{2}\right) \sim\left(r_{*} \tilde{r}_{1} s_{1}, r_{*} \tilde{r}_{1} m_{1}+\right.$ $\left.r_{*} \tilde{r}_{2} m_{2}\right) \sim\left(\tilde{r}_{1} s_{1}, \tilde{r}_{1} m_{1}+\tilde{r}_{2} m_{2}\right)$. By symmetry, we have the same independence for choice of $\left(s_{2}, m_{2}\right)$. Finally, suppose that instead of choosing $\tilde{r}_{1}, \tilde{r}_{2}$ we choose $\tilde{b}_{1}, \tilde{b}_{2}$. As $\tilde{r}_{1} s_{1} \in S$. By the combined Ore condition we may choose $s_{\sharp} \in S, r_{\sharp} \in R$, such that $r_{\sharp} \tilde{r}_{1}=s_{\sharp} \tilde{b}_{1}$ with $r_{\sharp} \tilde{r}_{1} s_{1}=s_{\sharp}\left(\tilde{b}_{1} s_{1}\right) \in S$. Hence $\left(\tilde{r}_{1} s_{1}, \tilde{r}_{1} m_{1}+\tilde{r}_{2} m_{2}\right) \sim\left(r_{\sharp} \tilde{r}_{1} s_{1}, r_{\sharp} \tilde{r}_{1} m_{1}+\right.$ $\left.r_{\sharp} \tilde{r}_{2} m_{2}\right) \sim\left(s_{\sharp} \tilde{b}_{1} s_{1}, s_{\sharp} \tilde{b}_{1} m_{1}+r_{\sharp} \tilde{r}_{2} m_{2}\right)$. Now $r_{\sharp} \tilde{r}_{2} s_{2}=r_{\sharp} \tilde{r}_{1} s_{1}=s_{\sharp} \tilde{b}_{1} s_{1}=$ $s_{\sharp} \tilde{b}_{2} s_{2}$, hence by left reversibility, there is $s_{2}^{*}$ such that $s_{2}^{*} r_{\sharp} \tilde{r}_{2}=s_{2}^{*} s_{\sharp} \tilde{b}_{2}$. Thus $\left(s_{\sharp} \tilde{b}_{1} s_{1}, s_{\sharp} \tilde{b}_{1} m_{1}+r_{\sharp} \tilde{r}_{2} m_{2}\right) \sim\left(s_{2}^{*} s_{\sharp} \tilde{b}_{1} s_{1}, s_{2}^{*} s_{\sharp} \tilde{b}_{1} m_{1}+s_{2}^{*} s_{\sharp} \tilde{b}_{2} m_{2}\right) \sim$ $\left(\tilde{b}_{1} s_{1}, \tilde{b}_{1} m_{1}+\tilde{b}_{2} m_{2}\right)$, as required.

(ii) In the proof of existence in (i) we have seen that + is commutative. Notice also that the class of $(s, 0)$ (independent on $S$ ) is the neutral element. For any pair, and hence for any triple of elements in $S \times M / \sim$, we can choose representatives such that all three are of the form $(s, m)$ with the same $s$. For such triples the associativity directly follows by applying (10).

(iii) As $p$ and the projection $S \times R \times M \rightarrow S \times M \rightarrow(S \times M) / \sim$ are additive it is sufficient to show that $p$ sends the kernel of the projection to $0 \in(S \times M) / \sim$. The kernel of the projection is spanned by the elements of several obvious types, so we check for generators.

1. $(s, r, m)-\left(s^{\prime}, r^{\prime}, m\right)$ where $s^{-1} r=\left(s^{\prime}\right)^{-1} r^{\prime}$. That means that for some $\tilde{s} \in S, \tilde{r} \in R$ we have $\tilde{s} s=\tilde{r} s^{\prime}$ and $\tilde{s} r=\tilde{r} r^{\prime}$. Compute $p(s, r, m)-p\left(s^{\prime}, r^{\prime}, m^{\prime}\right)=(s, r m)+\left(s^{\prime},-r^{\prime} m^{\prime}\right)=(\tilde{s} s, \tilde{s} r m)+$ $\left(\tilde{r} s^{\prime},-\tilde{r} r^{\prime} m^{\prime}\right)=\left(\tilde{s} s, \tilde{s} r m-\tilde{r} r^{\prime} m^{\prime}\right)=0$.

2. Elements $\left(s, r+r^{\prime}, m\right)-(s, r, m)-\left(s, r+r^{\prime}, m\right)$, as well as $\left(s, r r^{\prime}, m\right)-\left(s, r, r^{\prime} m\right)$ and $\left(s, r, m+m^{\prime}\right)-(s, r, m)-\left(s, r, m^{\prime}\right)$ go to 0 because, by (ii), in computing $p$ one has to first act with the second component to the third.

7.4 Proposition. $(S \times M) / \sim$ is additively canonically isomorphic to $S^{-1} M$. This isomorphism equips $(S \times M) / \sim$ with the canonical left $S^{-1} R$-module structure for which the following formulas can be taken as defining:

$$
t^{-1} r(s, m)=\left(s_{*} t, r_{*} m\right) \in(S \times M) / \sim
$$

where $s_{*} \in S$ and $r_{*} \in R$ such that $s_{*} r=r_{*} s$.

Proof. By the lemma we have the first statement and hence we can view the class of $(s, m)$ as $s^{-1} \otimes m$ and the formulas follow. They are 
defining because the formulas agree with the action transferred by an isomorphism, hence the existence, and by the left Ore condition for any $t^{-1} r$ and $(s, m)$ there are $s_{*}, r_{*}$ qualifying for the formulas, hence the uniqueness.

7.4a Corollary. Let $S \subset R$ be left Ore and $M$ a left $R$-module. Then $m=0$ for some $s$ iff $\exists s \in S$ and $s m=0$.

7.4b (long) Exercise. Let $S, W$ be two left Ore sets in $R$, and $M={ }_{R} M$ a left $R$-module. The relation $\sim$ on $S \times W \times M$ given by

$$
(s, w, m) \sim\left(s^{\prime}, w^{\prime}, m^{\prime}\right) \Leftrightarrow\left\{\begin{array}{l}
\exists r, r^{\prime}, u, u^{\prime}, \tilde{r}, \tilde{r}^{\prime} \in R, \exists \tilde{w}, \tilde{w}^{\prime} \in W, \\
r s=r^{\prime} s^{\prime} \in S, u \tilde{w}=u^{\prime} \tilde{w}^{\prime} \in W, \\
\tilde{w} r=\tilde{r} w, \tilde{w}^{\prime} r^{\prime}=\tilde{r}^{\prime} w^{\prime}, z \tilde{r} m=z^{\prime} \tilde{r}^{\prime} m^{\prime}
\end{array}\right.
$$

is an equivalence relation. Map $(S \times W \times M / \sim) \rightarrow S^{-1} W^{-1} M$ given by $(s, w, m) \mapsto s^{-1} \otimes w^{-1} \otimes m$ is well-defined and bijective.

\section{Monads, comonads and gluing}

8.1 A monoidal category is a category $\mathcal{C}$ equipped with a 'tensor' product bifunctor $\otimes: \mathcal{C} \times \mathcal{C} \rightarrow \mathcal{C}$; a distinguished object $1_{\mathcal{C}}$, a family of associativity isomorphisms $c_{A B C}:(A \otimes B) \otimes C \rightarrow A \otimes(B \otimes C)$, natural in objects $A, B, C$ in $\mathcal{C}$; left unit $l_{A}: 1_{\mathcal{C}} \otimes A \rightarrow A$, and right unit isomorphisms $r_{A}: A \otimes 1_{\mathcal{C}} \rightarrow A$, both indexed by and natural in objects $A$ in $\mathcal{C}$; furthermore, require some standard coherence conditions (pentagon axiom for associativity coherence; left and right unit coherence conditions, cf. [17, 71]). A monoidal category $\left(\tilde{\mathcal{C}}, \otimes, 1_{\mathcal{C}}, c, r, l\right)$ is strict if $c_{A B C}, l_{A}, r_{A}$ are actually all identity morphisms.

8.2 Monads and comonads. Given a diagram of categories $\mathcal{A}, \mathcal{B}, \mathcal{C}$, functors $f_{1}, f_{2}, g_{1}, g_{2}$ and natural transformations $F, G$ as follows

$$
\mathcal{A} \underset{f_{2}}{\stackrel{f_{1}}{\longrightarrow}} \mathcal{B} \underset{g_{2}}{\stackrel{g_{1}}{\longrightarrow}} \mathcal{C}
$$

one defines the natural transformation $G \star F: g_{2} \circ f_{2} \Rightarrow g_{1} \circ f_{1}$ by

$(G \star F)_{A}:=G_{f_{1}(A)} \circ g_{2}\left(F_{A}\right)=g_{1}\left(F_{A}\right) \circ G_{f_{2}(A)}: g_{2}\left(f_{2}(A)\right) \rightarrow g_{1}\left(f_{1}(A)\right)$.

$(F, G) \mapsto F \star G$ is called the Godement product ('horizontal composition', cf. (11)). It is associative for triples for which $F \star(G \star H)$ is defined. 
Given functors $f, g, h: \mathcal{A} \rightarrow \mathcal{B}$ and natural transformations $\alpha$ : $f \Rightarrow g, \beta: g \Rightarrow h$, define their 'vertical' (or ordinary) composition $\beta \circ \alpha: f \Rightarrow h$ to be their composition taken objectwise: $(\beta \circ \alpha)_{A}:=$ $\beta_{A} \circ \alpha_{A}: f(A) \rightarrow h(A)$.

Note the interchange law: $(\alpha \circ \beta) \star(\gamma \circ \delta)=(\alpha \star \gamma) \circ(\beta \star \delta)$.

If $T, T^{\prime}$ are endofunctors in $\mathcal{A}$ and $\alpha: T \Rightarrow T^{\prime}, \beta: T^{\prime} \Rightarrow T$ natural transformations, one may also use the concatenation notation: $\alpha T: T T \Rightarrow$ $T^{\prime} T$ is given by $(\alpha T)_{M}:=\alpha_{T M}: T(T M) \rightarrow T^{\prime}(T M)$, i.e. $\alpha T \equiv \alpha \star 1_{T}$; similarly, $T \alpha$ equals $1_{T} \star \alpha$, or, $(T \alpha)_{M}=T\left(\alpha_{M}\right): T T M \rightarrow T T^{\prime} M$. This notation extends to the sequences with more functors but only one natural transformation - it is preferable to specify product $\circ$ versus $\star$ among the words if each has some natural transformation mixed in. Here concatenation is higher binding than any of the composition signs. Notice $1_{T} \star 1_{S}=1_{T \circ S}$.

Given a monoidal category $\tilde{\mathcal{C}}:=\left(\mathcal{C}, \otimes, 1_{\tilde{\mathcal{C}}}\right)$ a monoid in $\mathcal{C}$ is a pair $(X, \mu)$ of an object $X$ and a multiplication morphism $\mu: X \otimes$ $X \rightarrow X$ which is associative and there is a 'unit' map $\eta: 1_{\tilde{\mathcal{C}}} \rightarrow X$ such that $\mu \circ(\eta \otimes$ id $)=\mu \circ($ id $\otimes \eta) \cong$ id (here the identification $1_{\tilde{\mathcal{C}}} \otimes X \cong X$ is assumed). As this characterizes the unit map uniquely, one may or may not include $\eta$ in the data, writing triples $(X, \mu, \eta)$ when convenient.

For a fixed small category $\mathcal{A}$, the category $\operatorname{End}_{\mathcal{A}}$ of endofunctors (as objects) and natural transformations (as morphisms) is a strict monoidal category: the product of endofunctors is the composition, the product of natural transformations is the Godement product, and the unit is $\operatorname{Id}_{\mathcal{A}}$.

A monad $(T, \mu, \eta)$ in an arbitrary category $\mathcal{A}$ is a monoid in $\operatorname{End}_{\mathcal{A}}$, and a comonad $(\perp, \delta, \eta)$ in $\mathcal{A}$ is a monad in $\mathcal{A}^{\text {op }}$. The natural transformations $\delta: \perp \perp \rightarrow \perp$ and $\epsilon: \perp \rightarrow \operatorname{Id}_{\mathcal{A}}$ are also called the coproduct and the counit of the comonad respectively.

An action of a monad $\mathbf{T}=(T, \mu, \eta)$ on an object $M$ in $\mathcal{A}$ is a morphism $\nu: T(M) \rightarrow M$ such that the diagram

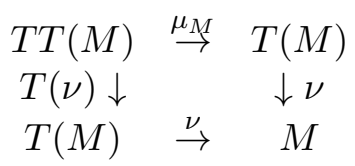

commutes and $\nu \circ \eta_{M}=\operatorname{Id}_{M}$. We say that $(M, \mu)$ is a module (older terminology: algebra) over T. A map $(M, \mu) \rightarrow(N, \nu)$ is a morphism $f: M \rightarrow N$ in $\mathcal{A}$ intertwining the actions in the sense that $f \circ \nu_{M}=\nu_{N} \circ T(f): T(M) \rightarrow N$. For a fixed $\mathbf{T}$, modules and 
their maps make a category $\mathcal{A}^{\mathbf{T}} \equiv \mathbf{T}-$ Mod, called the EilenbergMoore category of $\mathbf{T}$. The natural forgetful functor $U^{\mathbf{T}}: \mathcal{A}^{\mathbf{T}} \rightarrow \mathcal{A}$, $(M, \nu) \mapsto M$ is faithful, reflects isomorphisms and has a left adjoint $F: M \mapsto\left(M, \mu_{M}\right)$. The unit of adjunction $\eta: \operatorname{Id}_{\mathcal{A}} \Rightarrow U^{\mathbf{T}} F=T$ coincides with the unit of $\mathbf{T}$, and the counit $\epsilon: F U^{\mathbf{T}} \rightarrow \operatorname{Id}_{\mathcal{A}^{\mathbf{T}}}$ is given by $\epsilon_{(M, \nu)}=\nu$. The essential image of $F$, is a full and replete subcategory $\mathcal{A}_{\mathbf{T}} \subset \mathcal{A}^{\mathbf{T}}$ and it is called the Kleisli category of $\mathbf{T}$, while its objects are called free $\mathbf{T}$-modules.

Dually, for a comonad $\mathbf{G}=(G, \delta, \epsilon)$ in $\mathcal{A}$, a $\mathbf{G}$-comodule is an object in category $\mathbf{G}-$ Comod $:=\left(\left(\mathcal{A}^{\mathrm{op}}\right)^{\mathbf{G}}\right)^{\mathrm{op}}=(\mathbf{G}-\text { Mod })^{\mathrm{op}}$; equivalently it is a pair $(M, \sigma)$ where $M$ is an object in $\mathcal{A}$ and $\sigma: M \rightarrow G(M)$ is a morphism in $\mathcal{A}$ such that

$$
\begin{array}{ccc}
M & \stackrel{\sigma}{\rightarrow} & G(M) \\
\sigma \downarrow & & \downarrow G(\sigma) \\
G(M) & \stackrel{\delta}{\rightarrow} & G G(M)
\end{array}
$$

and $\epsilon_{M} \circ \sigma=\operatorname{Id}_{M} . \mathrm{A} \operatorname{map}\left(M, \rho_{M}\right) \rightarrow\left(N, \nu_{N}\right)$ is a morphism $f:$ $M \rightarrow N$ in $\mathcal{A}$ intertwining the coactions in the sense that $\sigma_{N} \circ f=$ $T(f) \circ \sigma_{M}: M \rightarrow T(N)$. The forgetful functor $\mathbf{G}-\operatorname{Mod} \rightarrow \mathcal{A}^{\text {op }}$ may be interpreted as a functor $U_{\mathbf{G}}: \mathbf{G}-\operatorname{Comod} \rightarrow \mathcal{A}$ which thus has a right adjoint $H: M \mapsto(G(M), \delta)$ whose essential image by definition consists of cofree G-comodules. The counit of the adjunction agrees with the counit of the comonad $\epsilon: G=U_{\mathbf{G}} H \Rightarrow \operatorname{Id}_{\mathcal{A}}$ and the unit $\eta: \operatorname{Id}_{\mathbf{G}-\text { Comod }} \Rightarrow H U_{\mathbf{G}}$ is given by $\eta_{(M, \sigma)}=\sigma:(M, \sigma) \rightarrow(G(M), \delta)$.

8.3 An archetypal example of a monad is constructed from a pair of adjoint functors $Q^{*} \dashv Q_{*}$ where $Q_{*}: \mathcal{B} \rightarrow \mathcal{A}$. In other words there are natural transformations $\eta: \operatorname{Id}_{\mathcal{A}} \Rightarrow Q_{*} Q^{*}$ called the unit and $\epsilon: Q^{*} Q_{*} \Rightarrow \operatorname{Id}_{\mathcal{B}}$, called the counit of the adjunction, such that the composites in the two diagrams

$$
Q_{*} \stackrel{\eta_{Q_{*}}}{\rightarrow} Q_{*} Q^{*} Q_{*} \stackrel{Q_{*}(\epsilon)}{\rightarrow} Q_{*}, \quad Q^{*} \stackrel{Q^{*}(\eta)}{\rightarrow} Q^{*} Q_{*} Q^{*} \stackrel{\epsilon_{Q_{*}}}{\rightarrow} Q^{*},
$$

are the identity transformations. Then $\mathbf{T}:=\left(Q_{*} Q^{*}, 1_{Q_{*}} \star \epsilon \star 1_{Q^{*}}, \eta\right)$ is a monad in $\mathcal{A}$. In other words, the multiplication is given by

$$
\mu_{M}=Q_{*}\left(\epsilon_{Q^{*}(M)}\right): Q_{*} Q^{*} Q_{*} Q^{*}(M)=T T(M) \rightarrow Q_{*} Q^{*}(M)=T(M) .
$$

The comparison functor $K^{\mathbf{T}}: \mathcal{B} \rightarrow \mathcal{A}^{\mathbf{T}}$ is defined by

$$
M \mapsto\left(Q_{*}(M), Q_{*}\left(\epsilon_{M}\right)\right), \quad F \mapsto Q_{*}(f) .
$$


It is full and $Q^{*}$ factorizes as $\mathcal{B} \stackrel{K^{\mathbf{T}}}{\rightarrow} \mathcal{A}^{\mathbf{T}} \stackrel{U^{\mathbf{T}}}{\rightarrow} \mathcal{A}$. More than one adjunction (varying $\mathcal{B}$ ) may generate the same monad in $\mathcal{A}$ in this vein.

Dually, $\mathbf{G}:=\left(Q^{*} Q_{*}, Q^{*} \eta Q_{*}, \epsilon\right)$ is a comonad, i.e. a monad in $\mathcal{B}^{\text {op }}$. The comparison functor $K^{\mathbf{G}}: \mathcal{A}^{\text {op }} \rightarrow\left(\mathcal{B}^{\text {op }}\right)^{\mathbf{T}}$ is usually identified with a 'comparison functor' $K_{\mathbf{G}}: \mathcal{A} \rightarrow\left(\left(\mathcal{B}^{\mathrm{op}}\right)^{\mathbf{T}}\right)^{\mathrm{op}} \equiv \mathbf{G}-$ Comod which is hence given by $N \mapsto\left(Q^{*}(N), \eta_{Q^{*}(N)}\right)$. $K_{\mathbf{G}}$ is full and $Q_{*}$ factorizes as $\mathcal{A} \stackrel{K_{\mathbf{G}}}{\rightarrow} \mathbf{G}-$ Comod $\stackrel{U_{\mathbf{G}}}{\rightarrow} \mathcal{B}$.

8.4 A map of monoids $f:(A, \mu, \eta) \rightarrow\left(A^{\prime}, \mu^{\prime}, \eta^{\prime}\right)$ in a monoidal category $\left(\mathcal{A}, \otimes, 1_{\mathcal{A}}, a, l, r\right)$ is a morphism $f: A \rightarrow A^{\prime}$ in $\mathcal{A}$, commuting with multiplication: $\mu \circ(f \otimes f)=f \circ \mu$; and with the unit map: $\eta^{\prime} \circ \mu=$ $\eta \otimes \eta$, where on the left the application of one of the isomorphisms $l_{1_{\mathcal{A}}}, r_{1_{\mathcal{A}}}: 1_{\mathcal{A}} \otimes 1_{\mathcal{A}} \rightarrow 1_{\mathcal{A}}$ is assumed. In particular, the morphism $\phi:(T, \mu, \eta) \rightarrow\left(T^{\prime}, \mu^{\prime}, \eta^{\prime}\right)$ of monads in $\mathcal{A}$ is a natural transformation $\phi: T \rightarrow T^{\prime}$ such that $\mu \circ(\phi \star \phi)=\phi \circ \mu: T T \Rightarrow T^{\prime}$ and $\eta^{\prime} \circ \mu=\eta \star \eta:$ $T T \Rightarrow \operatorname{Id}_{\mathcal{A}}$. If $M$ is an object in $\mathcal{A}$ and $\nu$ a $T^{\prime}$-action on $M$, then $\nu^{\prime} \circ \phi_{M}: T M \Rightarrow M$ is a $T$-action on $M$. More precisely, a natural transformation $\phi: T \Rightarrow T^{\prime}$ and rules $\mathcal{A}^{\phi}(M, \nu)=\left(M, \nu^{\prime} \circ \phi_{M}\right)$ and $\mathcal{A}^{\phi}(f)=f$ define a functor

$$
\mathcal{A}^{\phi}: \mathcal{A}^{\mathbf{T}^{\prime}} \rightarrow \mathcal{A}^{\mathbf{T}}
$$

iff $\phi$ is a morphism of monads and every functor $\mathcal{A}^{\mathbf{T}^{\prime}} \rightarrow \mathcal{A}^{\mathbf{T}}$ inducing the identity on $\mathcal{A}$ is of that form.

8.5 Let $\Delta$ be the 'simplicial' category: its objects are nonnegative integers viewed as finite ordered sets $\mathbf{n}:=\{0<1<\ldots<n\}$ and its morphisms are nondecreasing monotone functions. Given a category $\mathcal{A}$, denote by $\operatorname{Sim} \mathcal{A}$ the category of simplicial objects in $\mathcal{A}$, i.e. functors $F: \boldsymbol{\Delta}^{\mathrm{op}} \rightarrow \mathcal{A}$. Represent $F$ in $\operatorname{Sim} \mathcal{A}$ as a sequence $F_{n}:=$ $F(\mathbf{n})$ of objects, together with the face maps $\partial_{i}^{n}: F_{n} \rightarrow F_{n-1}$ and the degeneracy maps $\sigma_{i}^{n}: F_{n} \rightarrow F_{n+1}$ for $i \in \mathbf{n}$ satisfying the familiar simplicial identities [127, 71]. Notation $F_{\bullet}$ for this data is standard.

Given a comonad $\mathbf{G}$ in $\mathcal{A}$ one defines the sequence $\mathbf{G}_{\bullet}$ of endofunctors $\mathbb{Z}_{\geq 0} \ni n \mapsto \mathbf{G}_{n}:=G^{n+1}:=G \circ G \circ \ldots \circ G$, together with natural transformations $\partial_{i}^{n}: G^{i} \epsilon G^{n-i}: G^{n+1} \rightarrow G^{n}$ and $\sigma_{i}^{n}$ : $G^{i} \delta G^{n-i}: G^{n+1} \rightarrow G^{n+2}$, satisfying the simplicial identities. Use $\epsilon G \circ \delta=G \epsilon \circ \delta=\operatorname{Id}_{\mathcal{A}}$ in the proof. Hence any comonad $\mathbf{G}$ canonically induces a simplicial endofunctor, i.e. a functor $\mathbf{G}_{\bullet}: \Delta^{\mathrm{op}} \rightarrow \operatorname{End} \mathcal{A}$, or equivalently, a functor $\mathbf{G}_{\bullet}: \mathcal{A} \rightarrow \operatorname{Sim} \mathcal{A}$. The counit $\epsilon$ of the comonad G satisfies $\epsilon \circ \partial_{0}^{1}=\epsilon \circ \partial_{1}^{1}$, hence $\epsilon: \mathbf{G}_{\bullet} \rightarrow \operatorname{Id}_{\mathcal{A}}$ is in fact an augmented simplicial endofunctor. 
This is widely used in homological algebra ([71, 127, 102]), and now also in the cohomological study of noncommutative spaces (93]).

8.6 Barr-Beck lemma. ([6, [72]) Let $Q^{*} \dashv Q_{*}$ be an adjoint pair and $\mathbf{T}$ its associated monad (or, alternatively, $\mathbf{G}$ its associated comonad), constructed as in 8.3. Recall the notions of preserving and reflecting (co)limits from $\mathbf{1 . 6}$.

If $Q_{*}$ preserves and reflects coequalizers of all parallel pairs in $\mathcal{A}$ (for which coequalizers exists) and if any parallel pair mapped by $Q_{*}$ into a pair having a coequalizer in $\mathcal{B}$ has a coequalizer in $\mathcal{A}$ then the comparison functor $K: \mathcal{B} \rightarrow \mathcal{A}^{\mathbf{T}}$ is an equivalence of categories.

If $Q^{*}$ preserves and reflects equalizers of all parallel pairs in $\mathcal{B}$ (for which equalizers exists) and if any parallel pair mapped by $Q^{*}$ into a pair having an equalizer in $\mathcal{A}$ has an equalizer in $\mathcal{B}$ then the comparison functor $K^{\prime}: \mathcal{A} \rightarrow \mathbf{G}$ - Comod is an equivalence of categories.

Left (right) exact functors by the definition preserve finite limits (colimits) and faithful functors clearly reflect both; hence this is true for (co)equalizers of parallel pairs in particular. In abelian categories such (co)equalizers exist automatically. Hence

Corollary. Consider an adjoint pair $Q^{*} \dashv Q_{*}$ of additive functors between two abelian categories. If $Q_{*}$ is faithful and exact, then the comparison functor for the associated comonad is an equivalence. If $Q^{*}$ is faithful and exact then the comparison functor for the associated monad is an equivalence.

There are many characterizations when the comparison functor is an equivalence, e.g. Beck monadicity (=tripleability) theorem ([6, 8, [71, [72]), in various versions. Barr-Beck lemma gives only sufficient conditions, but it is easier to use, and still widely applicable.

8.7 (93]) A comonad associated to a family of continuous functors. Let $\left\{Q_{\lambda}^{*}: \mathcal{A} \rightarrow \mathcal{B}_{\lambda}\right\}_{\lambda \in \Lambda}$ be a small family of continuous (= having a right adjoint) functors. The categories $\mathcal{B}_{\lambda}$ are not necessarily constructed from $\mathcal{A}$ by a localization.

One may consider the category $\mathcal{B}_{\Lambda}:=\prod_{\lambda \in \Lambda} \mathcal{B}_{\lambda}$ whose objects are families $\prod_{\lambda \in \Lambda} M^{\lambda}$ of objects $M^{\lambda}$ in $\mathcal{B}_{\lambda}$ and morphisms are families $\prod_{\lambda \in \Lambda} f_{\lambda}: \prod_{\lambda \in \Lambda} M^{\lambda} \rightarrow \prod_{\lambda \in \Lambda} N^{\lambda}$ where $f_{\lambda}: M^{\lambda} \rightarrow N^{\lambda}$ is a morphism in $\mathcal{B}_{\lambda}$, with componentwise composition. This makes sense as the family of objects is literally a function from $\Lambda$ to the disjoint union $\coprod_{\lambda} \mathrm{Ob} \mathcal{B}_{\lambda}$ which is in the same Grothendieck universe. However, if the categories have some additional structure, it may be very nontrivial whether such structure can be extended to $\mathcal{B}_{\Lambda}$, and the answer may depend on the cardinality of $\Lambda$. 
The family of adjoint pairs $Q_{\lambda}^{*} \dashv Q_{\lambda *}$ defines an inverse image functor $\mathbf{Q}^{*}=\prod Q_{\lambda}^{*}: \mathcal{A} \rightarrow \mathcal{A}_{\Lambda}$ by $\mathbf{Q}^{*}(M):=\prod_{\lambda \in \Lambda} Q_{\lambda}^{*}(M)$ on objects and $\mathbf{Q}^{*}(f):=\prod_{\lambda} Q_{\lambda}^{*}(f)$ on morphisms. However, a direct image functor may not exist. We may naturally try $\mathbf{Q}_{*}: \prod_{\lambda}^{\prime} M^{\lambda} \mapsto \prod_{\lambda}^{\prime} Q_{*}\left(M^{\lambda}\right)$ where $\Pi^{\prime}$ is now the symbol for the Cartesian product in $\mathcal{A}$ which may not always exist. For finite families, with $\mathcal{A}$ abelian, these trivially exist. Assume a canonical choice of the Cartesian product, natural in the sense that the projections $p_{\nu\left\{M^{\lambda}\right\}_{\lambda}}^{\prime}: \prod_{\lambda} M^{\lambda} \rightarrow M^{\nu}$ are chosen to form a natural transformation of functors $p_{\nu}^{\prime}: \prod_{\lambda}^{\prime} \operatorname{Id}_{\mathcal{A}} \Rightarrow \mathrm{p}_{\nu}$ where $p_{\nu}: \mathcal{A}^{\Lambda} \rightarrow \mathcal{A}$ is the $\nu$-th formal projection from the power category $\mathcal{A}^{\Lambda}=\prod_{\lambda} \mathcal{A}$. The unique liftings $\boldsymbol{\eta}_{M}: M \rightarrow \mathbf{Q}_{*} \mathbf{Q}^{*}(M)$ of morphisms $\eta_{\nu M}: M \rightarrow Q_{\nu *} Q_{\nu}^{*}(M)$ in the sense that $(\forall \nu) \eta_{\nu M}=p_{\nu M}^{\prime} \circ \eta_{M}$ hence form a natural transformation $\boldsymbol{\eta}: \operatorname{Id}_{\mathcal{A}} \Rightarrow \mathbf{Q}_{*} \mathbf{Q}^{*}$.

On the other hand, for the families define $\epsilon \equiv \prod_{\lambda} \epsilon_{\lambda}: \prod_{\lambda} Q_{\lambda}^{*} Q_{\lambda *} \Rightarrow$ $\prod_{\lambda} \operatorname{Id}_{\mathcal{B}_{\lambda}}=\operatorname{Id}_{\mathcal{B}_{\Lambda}}$ componentwise. This way we obtain an adjunction $\mathbf{Q}^{*} \dashv \mathbf{Q}_{*}$. If $Q_{\lambda *}$ is faithful and exact for every $\lambda$ then $\mathbf{Q}_{*}$ is as well.

Consider the comonad $\mathbf{G}$ in $\mathcal{B}_{\Lambda}$ associated to $\mathbf{Q}^{*} \dashv \mathbf{Q}_{*}$. We are interested in situation when the comparison functor $K_{\mathbf{G}}$ is an equivalence of categories. That type of a situation arises in practice in two different ways:

- 1) All categories $\mathcal{A}, \mathcal{B}_{\lambda}$ and flat localization functors $Q_{\lambda}^{*}, Q_{\lambda *}$ are given at start and the construction is such that we know the faithfulness of $\mathbf{Q}_{*}$. good properties including that $\left\{Q_{\lambda}^{*}\right\}_{\lambda \in \Lambda}$ covers $\mathcal{A}$.

- 2) Only categories $\mathcal{B}_{\lambda}$ are given (not $\mathcal{A}$ ) but equipped with gluing morphisms i.e. the family $\Phi$ of flat functors (not necessarily localizations) $\phi_{\lambda, \lambda^{\prime}}^{*}: \mathcal{B}_{\lambda} \rightarrow \mathcal{B}_{\lambda^{\prime}}$ for each pair $\lambda, \lambda^{\prime}$, where $\Phi$ satisfies some cocycle condition.

8.7a In 1), to ensure the faithfulness of $\mathbf{Q}_{*}$ we require that the family $\left\{Q_{\lambda}^{*}\right\}_{\lambda \in \Lambda}$ is a flat cover of $\mathcal{A}$. That means that this is a small flat family of functors with domain $\mathcal{A}$ which is conservative, i.e. a morphism $f \in \mathcal{A}$ is invertible iff $Q_{\lambda}^{*}(f)$ is invertible for each $\lambda \in \Lambda$. A flat map whose direct image functor is faithful is called almost affine. In particular, this is true for adjoint triples $f^{*} \dashv f_{*} \dashv f^{!}$coming from a map $f: R \rightarrow S$ of rings. Adjoint triples where the direct image functor is faithful are called affine morphisms.

8.7b In 2), we a posteriori construct $\mathcal{A}$ to be $\mathcal{B}_{\Lambda}$ as before but equipped with functors $Q_{\lambda}^{*} \dashv Q_{\lambda *}$ constructed from $\phi$-functors. The cocycle condition for gluing morphism is equivalent to the associativity 
of the associated comonad ([17]). The remaining requirements are made to ensure that the comparison functor is an equivalence and the other original data may be reconstructed as well. The EilenbergMoore category of the associated monad may be constructed directly from gluing morphisms, and it appears to be just a reformulation of the descent category. In a generalization, the category $\mathcal{B}_{\lambda \lambda^{\prime}}$ which is the essential image of $\phi_{\lambda \lambda^{\prime}}$ in $\mathcal{B}_{\lambda^{\prime}}$ may be replaced by any 'external' category $\mathcal{B}_{\lambda \lambda^{\prime}}$, but then, instead of $\phi_{\lambda \lambda^{\prime}}^{*}$ one requires not only flat functors $\phi_{\lambda \lambda^{\prime}}^{*}=: \phi_{\lambda \lambda^{\prime}}^{\lambda *}: \mathcal{B}_{\lambda} \rightarrow \mathcal{B}_{\lambda \lambda^{\prime}}$, but also flat functors $\tilde{\phi}_{\lambda \lambda^{\prime}}^{*}:=\phi_{\lambda \lambda^{\prime}}^{\lambda^{\prime} *}:$ $\mathcal{B}_{\lambda^{\prime}} \rightarrow \mathcal{B}_{\lambda \lambda^{\prime}}$. This generalization is essentially more general only if we allow the direct image functors (of the second type, i.e. $\phi_{\lambda \lambda^{\prime} *}^{\lambda^{\prime}}$ ), to be not necessarily fully faithful (hence $\mathcal{B}_{\lambda \lambda^{\prime}}$ may not be viewed as a full subcategory of $\mathcal{B}_{\lambda^{\prime}}$ ). Another generalization of this descent situation, which can be phrased as having a pseudofunctor from a finite poset $\Lambda$ (viewed as a 2-category with only identity 2-cells) to the 2-category of categories has been studied by V. LunTs (65]). This analogue of descent category is called a configuration category.

8.7c The usual formalism of descent is via fibred categories, cf. [125]. For the correspondence between the two formalisms see, e.g. 94]).

8.8 Globalization lemma. (version for Gabriel filters: in 96] p. 103) Suppose $\left\{Q_{\lambda}^{*}: R-\operatorname{Mod} \rightarrow \mathcal{M}_{\lambda}\right\}_{\lambda \in \Lambda}$ is a finite cover of $R$ - Mod by flat localization functors (e.g. a conservative family of Ore localizations $\left.\left\{S_{\lambda}^{-1} R\right\}_{\lambda \in \Lambda}\right)$. Denote $Q_{\lambda}:=Q_{\lambda *} Q_{\lambda}^{*}$ where $Q_{\lambda *}$, is the right adjoint to $Q_{\lambda}^{*}$. Then for every left $R$-module $M$ the sequence

$$
0 \rightarrow M \stackrel{\iota_{\Lambda, M}}{\longrightarrow} \prod_{\lambda \in \Lambda} Q_{\lambda} M \stackrel{\iota_{\Lambda \Lambda, M}}{\longrightarrow} \prod_{(\mu, \nu) \in \Lambda \times \Lambda} Q_{\mu} Q_{\nu} M
$$

is exact, where $\iota_{\Lambda M}: m \mapsto \prod \iota_{\lambda, M}(m)$, and

$$
\iota_{\Lambda \Lambda M}:=\prod_{\lambda} m_{\lambda} \mapsto \prod_{(\mu, \nu)}\left(\iota_{\mu, \nu, M}^{\mu}\left(m_{\mu}\right)-\iota_{\mu, \nu, M}^{\nu}\left(m_{\nu}\right)\right) .
$$

Here the order matters: pairs with $\mu=\nu$ may be (trivially) skipped, but, unlike in the commutative case, we can not confine to the pairs of indices with $\mu<\nu$ only. Nota bene!

Proof. A direct corollary of Barr-Beck lemma. For proofs in terms of Gabriel filters and torsion see [97, pp. 23-25, and [52, 120, 121].

8.9 A monad $\mathbf{T}=(T, \mu, \eta)$ in $\mathcal{A}$ is idempotent if the multiplication $\mu: T T \Rightarrow T$ is an equivalence of endofunctors. As $\mu_{M}$ is the left 
inverse of $\eta_{T M}$, and of $T\left(\eta_{M}\right)$, then $\mu_{M}$ is invertible iff any of them is, hence both, and then $\eta_{T M}=T\left(\eta_{M}\right)=\mu_{M}^{-1}$.

If $\nu: T M \rightarrow M$ is a $\mathbf{T}$-action, then by naturality $\eta_{M} \circ \nu=$ $T(\nu) \circ \eta_{T M}=T(\nu) \circ T\left(\eta_{M}\right)=T\left(\nu \circ \eta_{M}\right)=\operatorname{Id}_{T M}$, hence $\eta_{M}$ is 2 -sided inverse of $\nu$ in $\mathcal{A}$, hence every $\mathbf{T}$-action is an isomorphism. Conversely, If every $\mathbf{T}$-action is an isomorphism, $\mu_{M}$ is in particular, and $\mathbf{T}$ is idempotent. Moreover, if every action $\nu: T M \rightarrow M$ is an isomorphism, then its right inverse $\eta_{M}$ must be the 2-sided inverse, hence there may be at most one action on a given object $M$ in $\mathcal{A}$. By naturality of $\eta$, its inverse $\nu$ which is well-defined on the full subcategory of $\mathcal{A}$ generated by objects in the image of $U^{\mathbf{T}}: \mathcal{A}^{\mathbf{T}} \rightarrow \mathcal{A}$, is also natural, i.e. it intertwines the actions, hence it is in fact a morphism in $\mathcal{A}^{\mathbf{T}}$, hence the forgetful functor $U^{\mathbf{T}}: \mathcal{A}^{\mathbf{T}} \rightarrow \mathcal{A}$ is not only faithful but also full. Its image is replete (closed under isomorphisms) as the existence of $\mathbf{T}$-actions on $M$ depends only on the isomorphism class of $M$ in $\mathcal{A}$.

In other words, $\mathcal{A}^{\mathbf{T}}$ includes via $U^{\mathbf{T}}$ as a full and replete subcategory into $\mathcal{A}$ and the inclusion has a left adjoint $F^{\mathbf{T}}$. In general, a (co)reflective subcategory $\mathcal{B} \hookrightarrow \mathcal{A}$ is a full and replete subcategory, such that the inclusion $U: \mathcal{B} \hookrightarrow \mathcal{A}$ has a (right) left adjoint, say $F$. As $F^{\mathbf{T}} \dashv U^{\mathbf{T}}$, we have just proved that $\mathcal{A}^{\mathbf{T}}$ is canonically isomorphic to a reflective subcategory of $\mathcal{A}$ via inclusion $U^{\mathbf{T}}$ if $\mathbf{T}$ is idempotent. On the other hand, it may be shown that for any reflective subcategory $U: \mathcal{B} \hookrightarrow \mathcal{A}$ the corresponding monad $\left(U F, U\left(\epsilon_{F}\right), \eta\right)$ is idempotent and the comparison functor $\mathcal{K}: \mathcal{B} \cong \mathcal{A}^{\mathbf{T}}$ is an isomorphism. Similarly, coreflective subcategories are in a natural correspondence with idempotent comonads (cf.8.6).

\section{Distributive laws and compatibility}

9.1 A distributive law from monad $\mathbf{T}=\left(T, \mu^{T}, \eta^{T}\right)$ to monad $\mathbf{P}=\left(P, \mu^{P}, \eta^{P}\right)$ in $\mathcal{A}$ a (or "of $\mathbf{T}$ over $\mathbf{P}$ ") is a natural transformation $l: T P \Rightarrow P T$ such that

$$
\begin{array}{ll}
l \circ T\left(\eta^{P}\right)=\left(\eta^{P}\right)_{T}, & l \circ T\left(\mu^{P}\right)=\left(\mu^{P}\right)_{T} \circ P(l) \circ l_{P}, \\
l \circ\left(\eta^{T}\right)_{P}=P\left(\eta^{T}\right), & l \circ\left(\mu^{T}\right)_{P}=P\left(\mu^{T}\right) \circ l_{T} \circ T(l) .
\end{array}
$$

For clarity, we show the commutative diagram for one of these rela- 
tions.

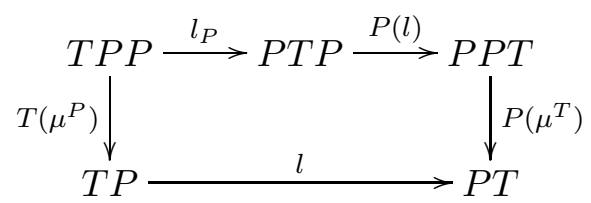

Then $\mathbf{P}$ lifts to a unique monad $\mathbf{P}^{\mathbf{T}}=\left(P^{\mathbf{T}}, \tilde{\mu}, \tilde{\eta}\right)$ in $\mathcal{A}^{\mathbf{T}}$, in the sense that $U^{\mathbf{T}} P^{\mathbf{T}}=P U^{\mathbf{T}}$ and for all $M \in \mathcal{A}^{T}$ we have $U^{\mathbf{T}}\left(\tilde{\eta}_{M}\right)=$ $T\left(\eta^{P}\right)_{U \mathbf{T}_{M}}$ and $U^{\mathbf{T}}\left(\tilde{\mu}_{M}\right)=T\left(\mu^{P}\right)_{U \mathbf{T}_{M}}$. Indeed, the endofunctor $P^{\mathbf{T}}$ is given by $(M, \nu) \mapsto(P M, \tilde{\nu})$ where $\tilde{\mu}_{(M, \nu)}=P(\nu) \circ l_{M}: T P M \rightarrow P M$.

Distributive laws from $\mathbf{T}$ to $\mathbf{P}$ are in a bijective correspondence with those monads in $\mathcal{A}$ whose underlying functor is $P T$ and whose unit is $\eta_{P} \star \eta_{T}$. In this correspondence, $\mu_{M}^{P T}:$ PTPTM $\rightarrow$ PTM is obtained by $\mu_{M}^{P T}=\left(\mu^{P} \star \mu^{T}\right) \circ P\left(l_{T M}\right)$, and conversely, $l_{M}$ by composition TPM $\stackrel{T P\left(\eta_{M}\right)}{\rightarrow} T P T M \stackrel{\eta_{T P F M}}{\rightarrow}$ PTPTM $\stackrel{\mu_{M}^{P T}}{\rightarrow} P T M$.

9.1a Distributive law from a comonad $\mathbf{G}=\left(G, \delta^{G}, \epsilon^{G}\right)$ to a comonad $\mathbf{F}=\left(F, \delta^{F}, \epsilon^{F}\right)$ is a natural transformation $l: F \circ G \Rightarrow G \circ F$ such that

$$
\begin{array}{ll}
G\left(\epsilon^{F}\right) \circ l=\left(\epsilon^{F}\right)_{G}, & \left(\delta^{G}\right)_{F} \circ l=G(l) \circ l_{G} \circ F\left(\delta^{G}\right), \\
\left(\epsilon^{G}\right)_{F} \circ l=F\left(\epsilon^{G}\right), & G\left(\delta^{F}\right) \circ l=l_{F} \circ F(l) \circ\left(\delta^{F}\right)_{G} .
\end{array}
$$

9.1b Mixed distributive law from a monad $\mathrm{T}$ to a comonad G: a natural transformation $l: T G \Rightarrow G T$ such that

$$
\begin{aligned}
\epsilon^{T} \circ l & =T(\epsilon), \quad l \circ \mu_{G}=G(\mu) \circ l_{T} \circ T(l), \\
l \circ \eta_{G} & =G(\eta), \quad l \circ T(\delta)=\delta_{T} \circ G(l) \circ l_{G} .
\end{aligned}
$$

It lifts the comonad $\mathbf{G}$ to a comonad $\mathbf{G}^{\mathbf{T}}$ in $\mathcal{A}^{\mathbf{T}}$, where $G^{\mathbf{T}}(M, \nu)=$ $\left(M G, G(\nu) \circ l_{M}\right)$.

9.1c Mixed distributive law from a comonad $\mathrm{G}$ to a monad $\mathbf{T}$ : a natural transformation $l: G T \Rightarrow T G$ such that

$$
\begin{array}{ll}
T(\epsilon) \circ l=\epsilon_{T}, & l \circ G(\mu)=\mu_{G} \circ T(l) \circ l_{T} \\
l \circ \eta_{G}=G(\eta), & T(\delta) \circ l=l_{G} \circ G(l) \circ \delta_{T} .
\end{array}
$$

9.2 Examples are abundant ([17] and [115, II). Categories with several structures over objects of some base category, have often imposed "compatibility" conditions which correspond to distributive laws. Consider, left $R$-modules and right $S$-modules, where the base category is the category $\mathbf{A b}$ of abelian groups. The rebracketing $(R \otimes$ ? $) \otimes S \mapsto$ $R \otimes(? \otimes S)$ is a distributive law from $R \otimes$ and $\otimes S$ in $\mathbf{A b}$, giving rise to 
a monad $U=(S \otimes ?) \circ(T \otimes$ ?), such that modules over $U$ are precisely $R-S$-bimodules. Similarly, one can describe $G$-equivariant versions of many standard categories of sheaves, modules, by considering one (co)monad for the underlying structure and another arising from a group (or Hopf algebra) action.

9.3 There is a notion of a monad and comonad in an arbitrary 2category ([115]) (our case is than in the 2-category of categories Cat). Thus for example, the compatibility of monoids and comonoids in an arbitrary monoidal category may be easily defined. Distributive laws between monoids and comonoids in $\mathrm{Vec}_{\mathbf{k}}$ are so-called entwining structures (21]). The Sweedler's crossed product in Hopf algebra theory is another special case (115],II).

9.4 Let $\mathbf{T}:=(T, \mu, \eta)$ be a monad, and $Q^{*}: \mathcal{A} \rightarrow \mathcal{B}$ a localization functor. The monad $\mathbf{T}$ is compatible with the localization if its underlying endofunctor $T$ is compatible with the localization, i.e. there is a functor $T_{\mathcal{B}}: \mathcal{B} \rightarrow \mathcal{B}$ with $Q^{*} T=T_{\mathcal{B}} Q^{*}$, cf. 3.5. In that case, $T_{\mathcal{B}}$ is the underlying endofunctor of a unique monad $\mathbf{T}_{\mathcal{B}}:=\left(T_{\mathcal{B}}, \mu^{\mathcal{B}}, \eta^{\mathcal{B}}\right)$ in $\mathcal{B}$ such that $\left(\mu^{\mathcal{B}}\right)_{Q^{*} N}=Q^{*}\left(\mu_{N}\right)$ for every $N$ in $\operatorname{Ob} \mathcal{A}$.

Proof. Let $f: N \rightarrow N^{\prime}$ be a morphism in $\mathcal{A}$, and $g: Q^{*} N^{\prime} \rightarrow M$ an isomorphism in $\mathcal{B}$. Consider the diagram

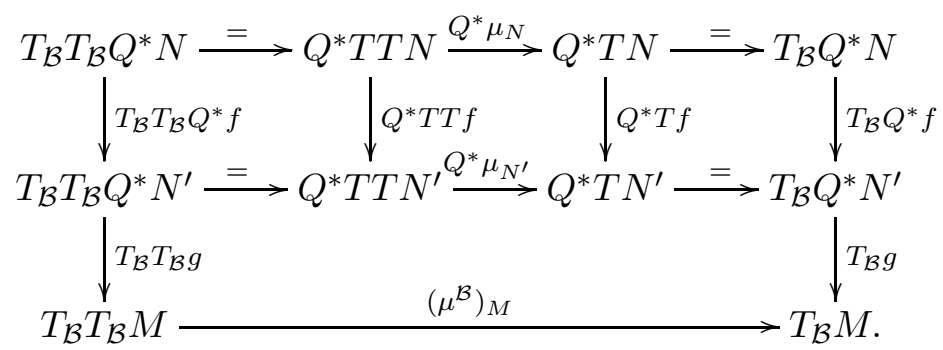

The upper part of the diagram clearly commutes. In particular, if $Q^{*} f$ is identity, then $Q^{*} \mu_{N}=Q^{*} \mu_{N^{\prime}}$. The vertical arrows in the bottom part are isomorphisms, so there is a map $\left(\mu^{\mathcal{B}}\right)_{M}$ filling the bottom line. One has to show that this map does not depend on the choices and that such maps form a natural transformation. The localization functor is a composition of a natural functor onto the quotient category and an equivalence. We may assume that $Q^{*}$ is the functor onto the quotient category. Then, by the construction of the quotient category, every morphism $g$ is of the zig-zag form as a composition of the maps of the form $Q^{*} f$ and formal inverses of such maps, and if $g$ is an isomorphism, both kinds of ingredients are separately invertible in $\mathcal{B}$. To show that $\left(\mu^{B}\right)_{M}=\left(T_{\mathcal{B}} g\right) \circ Q^{*}\left(\mu_{N^{\prime}}\right) \circ\left(T_{\mathcal{B}} T_{\mathcal{B}} g\right)^{-1}$ for every 
isomorphism $g: Q^{*} N^{\prime} \rightarrow M$ is the consistent choice, we use the upper part of the diagram repeatedly (induction by the length of zig-zag) for the zig-zag isomorphism $h=\left(g_{1}\right)^{-1} g_{2}: Q^{*} N_{2} \rightarrow Q^{*} N_{1}$ where $g_{i}: Q^{*} N_{i} \rightarrow M$. One obtains $T_{\mathcal{B}} h \circ Q^{*} \mu_{N_{2}} \circ\left(T_{\mathcal{B}} T_{\mathcal{B}} h\right)^{-1}=Q^{*} \mu_{N_{1}}$, hence $\left(T_{\mathcal{B}} g_{2}\right) \circ Q^{*}\left(\mu_{N_{2}}\right) \circ\left(T_{\mathcal{B}} T_{\mathcal{B}} g_{2}\right)^{-1}=\left(T_{\mathcal{B}} g_{1}\right) \circ Q^{*}\left(\mu_{N_{1}}\right) \circ\left(T_{\mathcal{B}} T_{\mathcal{B}} g_{1}\right)^{-1}$.

Upper part of the diagram also shows the naturality for $\mu^{\mathcal{B}}$ with respect to each arrow of the form $Q^{*} f$ and with respect to formal inverses of such. For any morphism $h: M \rightarrow M^{\prime}$ in $\mathcal{B}$, using its zigzag representation, we extend this to the naturality diagram $\left(\mu^{\mathcal{B}}\right)_{M^{\prime}}$ 。 $T_{\mathcal{B}} T_{\mathcal{B}} h=T_{\mathcal{B}} T_{\mathcal{B}} h \circ\left(\mu^{\mathcal{B}}\right)_{M}$. Uniqueness of $\mu^{\mathcal{B}}$ is clear by the requirement $\left(\mu^{\mathcal{B}}\right)_{Q^{*} N}=Q^{*}\left(\mu_{N}\right)$ and the naturality. The unit morphism $\eta: 1_{\mathcal{B}} \rightarrow$ $T_{\mathcal{B}}$ satisfies $g \circ \eta_{M}^{\mathcal{B}}=T_{\mathcal{B}}(g) \circ Q^{*}\left(\eta_{N}\right)$ for every isomorphism $g: Q^{*} N \rightarrow$ $M$ in $\mathcal{B}$ such that $N \in \mathrm{Ob} \mathcal{A}$. In particular, $\left(\eta^{\mathcal{B}}\right)_{Q^{*} N}=Q^{*}\left(\eta_{N}\right)$. The very axioms of a monad may be checked in a similar vein.

9.4b If $\mathbf{G}=(G, \delta, \epsilon)$ is a comonad and the endofunctor $G$ is compatible with each localization in family $\left\{Q_{\lambda}^{*}: \mathcal{A} \rightarrow \mathcal{B}_{\lambda}\right\}_{\lambda \in \Lambda}$, then there is a unique family of comonads $\left\{\mathbf{G}_{\lambda}=\left(G_{\lambda}, \delta_{\lambda}, \epsilon_{\lambda}\right)\right\}_{\lambda \in \Lambda}$ such that $Q_{\lambda}^{*} G=G_{\lambda} Q_{\lambda}^{*}$ for each $\lambda$. We have then $\left(\delta_{\lambda}\right)_{Q_{\lambda}^{*} M}=Q_{\lambda}^{*}\left(\delta_{M}\right)$ and $\left(\epsilon_{\lambda}\right)_{Q_{\lambda}^{*} M}=Q_{\lambda}^{*}\left(\epsilon_{M}\right)$ for every $M \in \mathrm{Ob} \mathcal{A}$.

9.5 If $Q^{*}: \mathcal{A} \rightarrow \mathcal{B}$ is a localization with right adjoint $Q_{*}$, and $T^{\prime}$ is an endofunctor in $\mathcal{B}$, then $Q_{*} T^{\prime} Q^{*}$ is compatible with $Q^{*}$. Indeed $\epsilon$ is an isomorphism by $\mathbf{3 . 4}$, hence $\epsilon_{T^{\prime} Q^{*}}: Q^{*} Q_{*} T^{\prime} Q^{*} \Rightarrow T^{\prime} Q^{*}$ is an isomorphism, and the assertion follows by $\mathbf{3 . 5}$.

9.6 Example from Hopf algebra theory. Let $B$ be a kbialgebra and $(E, \rho)$ a right $B$-comodule together with a multiplication $\mu: E \otimes_{\mathbf{k}} E \rightarrow E$ making it a $B$-comodule algebra, i.e. an algebra in the category of right $B$-comodules. The coaction $\rho: E \rightarrow E \otimes H$ is compatible with a fixed Ore localization $\iota: E \rightarrow S^{-1} E$ if there is a coaction $\rho_{S}: S^{-1} E \rightarrow S^{-1} E \otimes H$ which is an algebra map and such that $\rho_{S} \circ \iota=\left(\iota \otimes \operatorname{id}_{B}\right) \circ \rho . B$ induces a natural comonad $T=T(B, E)$ in $E$-Mod, such that $(E-\text { Mod })^{T}$ is a category of so-called $(E, B)$-Hopf modules. The compatibility above ensures that the localization lifts to a localization of $(E-\operatorname{Mod})^{T}([106], 8.5)$. Hence, $T$ is compatible with the localization in the usual sense, with numerous applications of this type of situation ([103, 104, 105, 106, 107]).

9.7 The compatibility of certain localizations of noncommutative spaces with differential functors is central in the treatment [66, 67, 68 69 of $\mathcal{D}$-modules on noncommutative spaces.

9.8 Distributive laws become a much simpler issue when both monads in question are idempotent in the sense of 8.9. In the local- 
ization literature this is roughly the situation treated under the name of mutual compatibility of localizations.

Let $S, W$ be two Ore sets. Then the set $S W$ of products $\{s w \mid s \in$ $S, w \in W\}$ is not necessarily multiplicatively closed.

Suppose $S W$ is multiplicatively closed. This means that $\forall s \in S$, $\forall w \in W$ if the product $w s$ is in $S W$ then $\exists w^{\prime} \in W, \exists s^{\prime} \in S$ such that $w s=s^{\prime} w^{\prime}$. Suppose now $M:={ }_{R} M \in R-\operatorname{Mod}$. Each element in $S^{-1} R \otimes_{R} W^{-1} R \otimes_{R} M$ is of the form $s^{-1} \otimes w^{-1} \otimes m$ with $s \in S$, $w \in W$ and $m \in M$. Suppose $S W$ is multiplicatively closed. Then $w s \in S W$, which by definition means that $\exists w^{\prime} \in W, \exists s^{\prime} \in S$ such that $w s=s^{\prime} w^{\prime}$. Choosing $s^{\prime}, w^{\prime}$ by this rule selects an assignment $s^{-1} \otimes w^{-1} \otimes m \mapsto\left(w^{\prime}\right)^{-1} \otimes\left(s^{\prime}\right)^{-1} \otimes m$. We claim that this assignment is well-defined and a map of left $R$-modules $S^{-1} R \otimes_{R} W^{-1} R \otimes_{R} M \rightarrow$ $W^{-1} R \otimes_{R} S^{-1} R \otimes_{R} M$. As $M$ runs through $R-$ Mod, such maps form a natural transformation $Q_{S} Q_{W} \rightarrow Q_{W} Q_{S}$ of functors, which is clearly an isomorphism.

In fact this natural transformation is a distributive law. Although the compatibility of $Q_{S}$ and $Q_{W}$ is symmetric, the converse does not hold: compatibility does not mean that $S W$ is multiplicatively closed. Indeed, let $R$ be a $\mathbb{C}$-algebra with two generators $a, b$ and relation $a b=q b a$ where $q \neq \pm 1,0$. Then the set multiplicatively generated by $A$ and set multiplicatively generated by $B$ are 2 -sided Ore sets, and the corresponding localization functors are compatible; however $A B$ is not multiplicatively closed.

If $S, W$ are left Ore in $R$, that does not mean that $\iota_{W}(S)$ is left Ore in $W^{-1} R$. Namely, the left Ore condition for (the image of) $S$ in $W^{-1} R$ includes the following: $\forall s \in S, \forall t \in T, \exists s^{\prime} \in S \exists\left(w^{\prime}\right)^{-1} r^{\prime} \in$ $W^{-1} R,\left(w^{\prime}\right)^{-1} r^{\prime} s=s^{\prime} w^{-1}$. If $R$ is a domain, this means that $r^{\prime} s w=$ $w^{\prime} s^{\prime}$. This is almost the same condition as that $S W$ is multiplicatively closed (above), except that one can choose extra $r^{\prime}$. In the same away as in the former case, we derive the compatibility of $Q_{S}$ and $Q_{W}$. If we change left Ore sets to right Ore sets, or $S$ being Ore in $W^{-1} R$ to $W$ being Ore in $S^{-1} R$ we get similar "Ore" equations $s w r=w^{\prime} s^{\prime}$, $w s r=s^{\prime} w^{\prime}$ etc. From the abstract point of view (say torsion theories) these compatibilities are indistinguishable.

The compatibility implies that the localization at the smallest multiplicative set generated by $S$ and $W$ is isomorphic to the consecutive localization by $S$ and then by $W$. This simplifies the formalism of localization (cf. semiseparated schemes and Cech resolutions of 93, cf. 8.8, [52] etc.). 


\section{Commutative localization}

Here we describe specifics in the commutative case, and further motivation from commutative algebraic geometry, and its abstractions.

10.1 Suppose $R$ is a unital (non)commutative ring, $Z(R)$ its center, and $S \subset Z(R)$ a multiplicative subset. Obviously, $S$ is automatically a left and right Ore subset in $R$, with simpler proofs for the construction and usage of the Ore localization. We say that $S^{-1} R=S \times R / \sim$ is the commutative localization of $R$ at $S$. The equivalence relation $\sim(\mathbf{4 . 1 3})$ simplifies to

$$
s^{-1} r \sim s^{\prime-1} r^{\prime} \Leftrightarrow \exists \tilde{s} \in S, \tilde{s}\left(s r^{\prime}-s^{\prime} r\right)=0 .
$$

Proof. By the definition, $\exists \tilde{s} \in S, \exists \tilde{r} \in R$, such that $\tilde{s} r=\tilde{r} r^{\prime}$ and $\tilde{s} s=\tilde{r} s^{\prime}$. Therefore,

$$
\tilde{s} s r^{\prime}=\tilde{r} s^{\prime} r^{\prime}=\tilde{r} r^{\prime} s^{\prime}=\tilde{s} r s^{\prime}=\tilde{s} s^{\prime} r .
$$

Unlike sometimes (mis)stated in the literature (e.g. [121, p.14), the commutative formula (12) (and variants of it) is inappropriate even for mildly noncommutative rings and even 2-sided Ore sets which are not in center. E.g. take the unital $\mathbb{C}$-algebra generated by two elements $b$ and $d$ with $b d=q d b$, where $\mathbb{C} \ni q \neq 1$. That algebra has no zero divisors. Let $S$ be the 2 -sided Ore set multiplicatively generated by $b$ and $d$. Formula $b^{-1}=(d b)^{-1} d$, and the criterion above would imply that $d b=b d$ with contradiction.

For general $R$ and $S$, formula (12) is actually not even an equivalence relation on $R \times S$.

10.2 General requirements on scheme-like theories. One wants to mimic several major points from the classical case. We first decide which geometric objects constitute make a category $\mathcal{C}$ of affine schemes; then find a suitable wider geometric category $\mathfrak{E} \mathfrak{s p}$ of spaces, in the sense that it is equipped with a fully faithful functor $\mathcal{C} \hookrightarrow \mathfrak{E s p}$, where the objects in the image will be called here geometric affine schemes; finally there is a gluing procedure which assigns to a collection $\left\{\mathfrak{C}_{h}\right\}_{h \in H}$ of geometric affine schemes and some additional 'gluing' data $Z$, a space which may be symbolically denoted by $\left(\coprod_{h} \mathfrak{C}_{h}\right) / Z \in$ $\mathfrak{E} \mathfrak{s p}$, together with canonical morphisms $\mathfrak{C}_{h} \rightarrow\left(\coprod_{h} \mathfrak{C}_{h}\right) / Z$ in $\mathfrak{E} \mathfrak{s p}$. For a fixed (type of) gluing procedure $\mathcal{G}$, and including all the isomorphic objects, one constructs this way a subcategory of locally affine spaces of type $(\mathcal{C}, \mathcal{G})$ in $\mathfrak{E} \mathfrak{s p}$. 
Additional requirements and typical choices are in place.

10.2a Abstract affine schemes. Most often one deals with some monoidal category $\tilde{\mathcal{A}}=\left(\mathcal{A}, \otimes, 1_{\tilde{A}}\right)$. Then $\mathcal{C}=\operatorname{Aff}(\tilde{A}):=(\operatorname{Alg}(\tilde{A}))^{\text {opp }}$ is the category of affine schemes in $\mathcal{A}$ i.e. the opposite to the category of algebras (monoids) in $\tilde{A}$. The basic example is the monoidal category of $R$-bimodules, where $R$ is a $\mathbf{k}$-algebra over a commutative ring $\mathbf{k}$. The affine schemes in this category are given by $\mathbf{k}$-algebra maps $f: R \rightarrow R^{\prime}$ (making such $R^{\prime}$ an $R$-bimodule).

If $\mathcal{A}$ is symmetric via a symmetry $\tau$ where $\tau_{A B}: A \otimes B \rightarrow B \otimes$ $A$, then one may consider only $\tau$-commutative algebras $(A, \mu, \eta)$, i.e. for which $\mu \circ \tau=\mu$. $\tau$-commutative affine schemes in $\mathcal{A}$ are the objects of the opposite of the category of $\tau$-commutative algebras $\mathcal{C}=\operatorname{cAff}(\tilde{A}, \tau):=(\operatorname{cAlg}(\tilde{A}, \tau))^{\text {opp }}$ (one often skips $\tau$ in the notation). Examples are (super)commutative affine schemes in a $\otimes$-category of $\mathbf{k}$-modules and also the opposite to the category $\underline{\operatorname{cdga}} \mathbf{k}$ of commutative differential graded $\mathbf{k}$-algebras, which is important in recent 'derived algebraic geometry' program.

10.2b Gluing for ringed topological spaces (and a version for local (1.) rings). A (locally) ringed space is a pair $(X, F)$ consisting of a topological space $X$, and a ('structure') sheaf $F$ of (l.) rings on $X$. A morphism of (1.) ringed spaces is a continuous map $f: X \rightarrow X^{\prime}$ with a comorphism i.e. a map of sheaves of (1.) rings $F^{\prime} \rightarrow f^{*} X^{\prime}$ over $X^{\prime}$. We obtain a category $\mathfrak{r} \mathfrak{S p}(\mathfrak{r} \mathfrak{S p})$. Given a full subcategory $\mathcal{A}$ of $\mathfrak{r} \mathfrak{S p}$, considered as a category of (heuristic term here) "local models" we may consider all (1.) ringed space $X$ for which there is a cover (in usual sense) $X=\cup X_{\alpha}$ of underlying topological spaces and for each $\alpha$ an isomorphism $X_{\alpha}^{0} \cong X_{\alpha}$ in $\mathfrak{r} \mathfrak{S p}$ (rSp) where $X_{\alpha}^{0}$ is in $\mathcal{A}$. More abstractly, but equivalently, consider all families of morphisms $\left\{i_{\alpha}: X_{\alpha} \rightarrow X\right\}_{\alpha}$, which are 'covers by embeddings': topologically covers of $X$ by families of monomorphisms (continuous, open and injective), and sheaf-wise isomorphisms on stalks. The intersections $X_{\alpha} \cap X_{\beta}$ represent fibred products $X_{\alpha} \times_{X} X_{\beta}$ in $\mathfrak{r} \mathfrak{S p}$. Spaces glued from objects in $\mathcal{A}$ are nothing but the colimits of the diagrams of the type $\coprod_{\alpha \beta} Y_{\alpha \beta} \Longrightarrow \amalg Y_{\alpha}$ where each of the morphisms $Y_{\alpha \beta} \rightarrow Y_{\alpha}$ and $Y_{\alpha \beta} \rightarrow Y_{\beta}$ are embeddings, and $Y_{\beta} \in \mathrm{Ob} \mathcal{A}$. There is a natural condition on $\mathcal{A}$ : each $X$ in $\mathcal{A}$ as a topological space has a basis of topology made out of (spaces of) some family of objects in $\mathcal{A}$ (or isomorphic to them); and this family may be chosen so that the restrictions of the structure sheaf agree. In the theory of schemes, affine schemes are such models: affine subschemes make a basis of topology, 
but not every open subset is affine; nor their intersections. Still the intersections and colimits exist from the start, in our ambient category of ringed spaces which is big enough.

10.2c Grothendieck (pre)topologies (G.(p)t.) and gluing in commutative algebraic geometry $([\mathbf{7 2}, 125])$. Schemes are glued from affine schemes in the Zariski topology (which may be considered both as an ordinary topology and a G.(p)t.); useful generalizations (e.g. algebraic spaces) in flat and étale G.(p)t. etc. A sieve is an assignment of a collection $J(R)$ of morphisms in $\mathcal{C}([72])$ with target $R$, such that if the target of $f$ is $J(R)$ and $g: R \rightarrow R^{\prime}$ then $g \circ f \in J\left(R^{\prime}\right)$. A G.t. is a collection of sieves $J(R)$ with target $R$ for each $R$ in $\mathrm{ObC}$, such that if $f \in J(R)$ and $g: R \rightarrow R^{\prime}$ then $\left.g \circ f \in J\left(R^{\prime}\right)\right)$, satisfying some axioms $(72])$. A G.pt. in a category $\mathcal{C}$ with fibred products is a class $\mathcal{T}$ of families $\left\{U_{\alpha} \rightarrow U\right\}_{\alpha}$ of morphisms (with one target per family), such that $\{\operatorname{Id}: U \rightarrow U\} \in \mathcal{T}$; if $\left\{f_{\alpha}: U_{\alpha} \rightarrow U\right\}_{\alpha} \in \mathcal{T}$ and $\forall \alpha\left\{g_{\alpha \beta}: U_{\alpha \beta} \rightarrow U_{\alpha}\right\}_{\beta} \in \mathcal{T}$ then $\left\{f_{\alpha} \circ g_{\alpha \beta}\right\}_{\alpha, \beta} \in \mathcal{T}$; and finally if $\left\{f_{\alpha}: U_{\alpha} \rightarrow U\right\}_{\alpha} \in \mathcal{T}$ and $g: V \rightarrow U$ is a morphism then $\left\{g^{*}\left(f_{\alpha}\right): V \times_{U} U_{\alpha} \rightarrow V\right\} \in \mathcal{T}$. Elements of $\mathcal{T}$ are called covers (in $\mathcal{T})$, and the pair $(\mathcal{C}, \mathcal{T})$ a site. To any (ordinary) topological space $X$ one associates a "small" site $\mathfrak{O} \mathfrak{u v}_{X}$ : objects are open subsets in $X$; morphisms are inclusions; $\left\{U_{\alpha} \hookrightarrow U\right\}_{\alpha}$ is a cover if $\cup_{\alpha} U_{\alpha}=U$.

A presheaf $F$ of sets on $X$ is a functor $F:\left(\operatorname{Ouv}_{X}\right)^{\text {op }} \rightarrow$ Sets; a presheaf $F$ on any site $(\mathcal{C}, \mathcal{T})$ with values in a category $\mathcal{D}$ with products is a functor $F: \mathcal{C}^{\mathrm{op}} \rightarrow \mathcal{D}$. Given a cover $\left\{U_{\alpha} \rightarrow U\right\}_{\alpha} \in$ $\mathcal{T}$, there are two obvious embeddings $\prod U_{\alpha} \times_{U} U_{\beta} \Longrightarrow \prod_{\alpha} U_{\alpha}$. A presheaf is a sheaf on $(\mathcal{C}, \mathcal{T})$ if $\left\{U_{\alpha} \rightarrow U\right\}_{\alpha} \in \mathcal{T}$ if for every such cover the induced diagram $F(U) \longrightarrow \prod_{\alpha} F\left(U_{\alpha}\right) \Longrightarrow \prod_{\alpha \beta} F\left(U_{\alpha} \times_{U} U_{\beta}\right)$ is an equalizer diagram in $\mathcal{D}$.

For gluing, one again needs some bigger ambient category (or, instead, some universal construction). Our local models are now (commutative) affine schemes Aff $:=\operatorname{Aff}(\mathrm{Ab})$ with a G.pt. $\mathcal{T}$. The Yoneda embedding $X \mapsto X^{\wedge}:=\operatorname{Aff}(?, X)$ is a fully faithful functor from Aff into the category PFas (Aff) of presheaves of sets on Aff. One typically deals with subcanonical G.t. which means that the presheaves in the Yoneda image (representable functors) are sheaves. As in the case of ordinary topologies, to construct the global locally $\mathcal{T}$-affine spaces, one needs colimits of certain diagrams of the form $\prod_{\alpha \beta} V_{\alpha \beta} \Longrightarrow \prod_{\alpha} U_{\alpha}$, where, in the addition, the colimit cone $\prod U_{\alpha} \rightarrow U$ corresponds to a cover in $\mathcal{T}$. As for example, the nonseparated schemes in Zariski 
topology, some locally $\mathcal{T}$-affine spaces may not be produced this way with $V_{\alpha \beta}$ being in Aff. Similar problems for biflat covers by localizations in noncommutative geometry are known (lack of compatibility of localizations; nonsemiseparated covers). Furthermore, one needs to extend the notion of $\mathcal{T}$-covers to the target category of sheaves. We hope that the reader sees at this point the meaning of this abstract machinery. We won't proceed with the full construction. Namely, in the commutative case, it is usually replaced by equivalent constructions. For example, to construct the algebraic spaces, one usually does not glue affine schemes "over intersections" but rather starts with an equivalence relation in category of schemes. In noncommutative case, an elaborated construction may be found in 94 and partly in [99. There is also an approach to G.t. and quasicoherent sheaves for noncommutative affine schemes by ORLOV (83]), utilizing ringed sites and sheaves of groupoids, where however the very construction of noncommutative locally affine spaces is not elaborated on.

10.3 Though we assume that the reader has been exposed to the commutative scheme theory, we here sketch the basic construction of an affine scheme, as a part of a widely-known easy generalization to pairs of the form (noncommutative ring, central subring), cf. 90].

A left ideal $\mathfrak{p} \subset R$ is prime if for any two left ideals $I, J$, if $I J \subset \mathfrak{p}$ then $I \subset \mathfrak{p}$ or $J \subset \mathfrak{p}$. A left ideal $\mathfrak{p} \subset R$ is completely prime if $f g \in \mathfrak{p}$ then $f \in \mathfrak{p}$ or $g \in \mathfrak{p}$; equivalently $R / \mathfrak{p}$ is a domain; or $R \backslash \mathfrak{p}$ is multiplicative. Each completely prime ideal is prime: otherwise one could find $f \in I \backslash \mathfrak{p}$ and $g \in J \backslash \mathfrak{p}$, such that $f g \in I J \subset \mathfrak{p}$ with contradiction. If $R$ is commutative the converse holds, as one can see by specializing the definitions to the principal left ideals $I=R f$, $J=R g, I J=R f R g=R f g$.

Consider the category $\mathcal{R}$, whose objects are pairs $(R, C)$, of a unital ring $R$ and a central subring $C \subset Z(R)$; and morphisms $(R, C) \rightarrow$ $\left(R^{\prime}, C^{\prime}\right)$ are maps of rings $\phi: R \rightarrow R^{\prime}$ such that $\phi(C) \subset C^{\prime}$.

Spec $C$ is the set of all prime ideals $\mathfrak{p}$ of $C$. For any ideal $I \subset C$, define $V(I) \subset \operatorname{Spec} C$ as the set of all $\mathfrak{p} \subset C$, such that $I \subset \mathfrak{p}$. Sets of the form $V(I)$ depend only (contravariantly with respect to inclusions) on the radical $\sqrt{I}$ (the intersection of all prime ideals containing $I$ ) and satisfy the axioms of antitopology. Complements of such sets hence form a topology on $\operatorname{Spec} C$, called Zariski topology. Principal open sets are the sets of the form $U_{f}=V((f))$, where $(f)$ is the (principal) ideal generated by $f \in C$. They make a basis of Zariski topology, i.e., any Zariski open set is a union of sets of that form. 
Open sets and inclusions form category $\mathfrak{O u v}_{C}$. Principal open sets and inclusions form its full subcategory $\mathfrak{O U v \mathfrak { P }}_{C}$.

Define $\mathcal{O}_{C}^{\prime}\left(U_{f}\right):=C\left[f^{-1}\right]$ and $\mathcal{O}_{R, C}^{\prime}\left(U_{f}\right):=R\left[f^{-1}\right]$. Every inclusion $U_{f} \hookrightarrow U_{g}$ induces the unital ring maps $\phi_{f, g, i}: \mathcal{O}_{i}^{\prime}\left(U_{g}\right) \rightarrow \mathcal{O}_{i}^{\prime}\left(U_{f}\right)$, $i \in\{C,(R, C)\}$. Hence we have contravariant functors $\mathcal{O}_{i}^{\prime}: \mathfrak{O u v P}_{C} \rightarrow$ Rings. Natural inclusions in $_{f}: C\left[f^{-1}\right] \rightarrow R\left[f^{-1}\right]$ form a natural

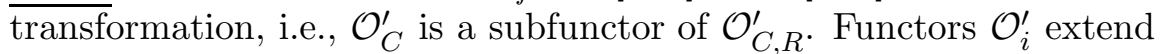
naturally to functors $\mathcal{O}_{i}: \mathfrak{O} \mathfrak{u v}_{C} \rightarrow$ Rings which are sheaves, and this requirement fixes sheaves $\mathcal{O}_{i}$ uniquely up to isomorphism of sheaves. Namely, represent any open set $U$ as the union $\cup U_{f}$ of a family $\mathcal{U}$ of (some or all) $U_{f} \subset U$. Define a diagram $\Delta(\mathcal{U})$ in $\underline{\text { Rings }}$ as follows. Vertices are of the form $\mathcal{O}_{i}\left(U_{f}\right)$ and of the form $\overline{\mathcal{O}_{i}\left(U_{f g}\right)}=\mathcal{O}_{i}\left(U_{f} \cap U_{g}\right)$ where $U_{f}, U_{g} \in \mathcal{U}$, and the arrows are $\phi_{f, f g, i}$. Then $\mathcal{O}_{i}(U)$ has to be isomorphic to the inverse limit of the diagram $\Delta(\mathcal{U})$ and may be consistently set so. Moreover the natural transformation in extends making $\mathcal{O}_{C}$ a subfunctor of $\mathcal{O}_{C, R}$. In fact, $\mathcal{O}_{C, R}$ is an algebra in the monoidal category of (2-sided) $\mathcal{O}_{C}$-modules. All the stalks $\left(\mathcal{O}_{C}\right)_{\mathfrak{p}}$ of $\mathcal{O}_{C}$ are local rings, namely the localizations $C_{\mathfrak{p}}:=C\left[\left\{f^{-1}\right\}_{f \notin \mathfrak{p}}\right]$ 'at prime ideal $\mathfrak{p}$ '.

Let $\psi: R \rightarrow R^{\prime}$ be a map of unital rings. The inverse image $\psi^{-1}(\mathfrak{p})$ of a completely prime ideal is completely prime. Let $\psi:(R, C) \rightarrow$ $\left(R^{\prime}, C^{\prime}\right)$ be a morphism in $\mathcal{R}$, and let map $\phi: C \rightarrow C^{\prime}$ agrees with $\psi$ on $C$. One has map $\phi^{*}: \operatorname{Spec} C^{\prime} \rightarrow \operatorname{Spec} C$ given by $\phi^{*}: \mathfrak{p} \mapsto$ $\phi^{-1}(\mathfrak{p})$. If $U \subset \operatorname{Spec} C^{\prime}$ is open, then $\phi^{-1}(U) \subset \operatorname{Spec} C$ is open as well, because $\phi^{*}(V(I))=V\left(\phi^{-1}(I)\right)$ for each ideal $I \subset C^{\prime}$. Hence, $\phi^{*}$ is continuous. If $g \notin \phi^{-1}(\mathfrak{p})$ then $\phi(g) \notin \mathfrak{p}$. Thus all elements $g \in C$, newly inverted in $C_{\phi^{-1}(\mathfrak{p})}$ (and $\left.R_{\phi^{-1}(\mathfrak{p})}\right)$ are also invertible in $C_{\mathfrak{p}}^{\prime}$ (and $\left.R_{\mathfrak{p}}^{\prime}\right)$. Hence, by the universality of localization, one has a unique map $\psi_{\mathfrak{p}}:\left(R_{\phi^{-1}(\mathfrak{p})}, C_{\phi^{-1}(\mathfrak{p})}\right) \rightarrow\left(R_{\mathfrak{p}}^{\prime}, C_{\mathfrak{p}}^{\prime}\right)$ such that $\psi_{\mathfrak{p}} \circ \iota_{\phi^{-1}(\mathfrak{p})}=\iota_{\mathfrak{p}} \circ \psi$. Define $\phi_{U}: \mathcal{O}_{R, C}\left(\phi^{*} U\right) \rightarrow \mathcal{O}_{R^{\prime}, C^{\prime}}(U)$ by $\left(\phi_{U}(r)\right)_{\mathfrak{p}}=\psi_{\mathfrak{p}}\left(r_{\phi^{-1}(\mathfrak{p})}\right)$. One has to check that $\phi_{U}(r)$ is indeed in $\mathcal{O}_{R^{\prime}, C^{\prime}}(U)$, i.e., that $\mathfrak{p} \mapsto \psi_{\mathfrak{p}}\left(r_{\phi^{-1}(\mathfrak{p})}\right)$ is indeed a section. For this consider $U_{f} \subset U$ affine, i.e., $U_{f}=\{\mathfrak{p} \mid f \notin \mathfrak{p}\}$ and $\phi^{*} U_{f}:=\left\{\phi^{-1}(\mathfrak{p}), \mathfrak{p} \not \supset f\right\}$. An argument as above gives map $\psi_{f}$ : $\mathcal{O}_{R, C}\left(\phi^{*} U_{f}\right) \rightarrow \mathcal{O}_{R^{\prime}, C^{\prime}}\left(U_{f}\right)$ satisfying $\psi_{f} \circ \iota_{\phi^{-1}\left(U_{f}\right)}=\iota_{U_{f}} \circ \psi$. It is easy to check then that $\psi_{f}$ induces $\psi_{\mathfrak{p}}$ in stalk over $\mathfrak{p} \in U_{f}$.

As a result, one obtains a map $\psi^{\sharp}: \phi_{*} \mathcal{O}_{R, C} \rightarrow \mathcal{O}_{R^{\prime}, C^{\prime}}$ of sheaves over $\operatorname{Spec} C^{\prime}$.

10.3a Let $\mathfrak{I S p}_{2}$ be a category whose object are locally ringed spaces $(X, \mathcal{O})$ in $\mathfrak{r} \mathfrak{s p}$ together with a sheaf $\mathcal{O}^{\text {nc }}$ of noncommutative algebras in the category of $\mathcal{O}$-modules. A morphism $\psi:\left(X, \mathcal{O}_{X}, \mathcal{O}_{X}^{\text {nc }}\right) \rightarrow$ 
$\left(Y, \mathcal{O}_{Y}, \mathcal{O}_{Y}^{\text {nc }}\right)$ in $\mathfrak{l s p}_{2}$ is a morphism $\psi^{\mathrm{c}}:\left(X, \mathcal{O}_{X}\right) \rightarrow\left(Y, \mathcal{O}_{Y}\right)$ in $\mathfrak{l} \mathfrak{S p}$, together with a map of sheaves of $\mathcal{O}_{X}$-modules $\psi^{\sharp}: \mathcal{O}_{Y}^{\text {nc }} \rightarrow \psi^{*} \mathcal{O}_{X}^{\text {nc }}$.

We have above constructed a functor $\mathrm{Spec}_{2}: \mathcal{R} \rightarrow \mathfrak{\mathfrak { S p } _ { 2 }}$.

10.3b In fact, $\mathcal{O}^{\text {nc }}$ is in a smaller category of quasicoherent sheaves of $\mathcal{O}$-modules (shortly: quasicoherent modules). A presheaf $F$ of $\mathcal{O}$ modules on a ringed space $(X, \mathcal{O})$ is quasicoherent (EGA 0.5.1.1) if $\forall x \in X \exists U^{\text {open }} \ni x$ with an exact sequence $\mathcal{O}^{I} \rightarrow \mathcal{O}^{J} \rightarrow F \rightarrow 0$ where $\mathcal{O}^{I}, \mathcal{O}^{J}$ are free $\mathcal{O}$-modules (of possibly infinite rank). If the ringed space is locally $\mathcal{T}$-affine for some Grothendieck topology $\mathcal{T}$ then one may take $U$ affine, and an equivalent definition of quasicoherence is that for any pair of affine open subsets $W \subset V \subset X$,

$$
F(W)=\mathcal{O}(W) \otimes_{\mathcal{O}(V)} F(V) .
$$

The noncommutative structure sheaf $\mathcal{O}^{\text {nc }}$ of $\operatorname{Spec}_{2}(R, C)$ is a quasicoherent $\mathcal{O}$-module. For presheaves of $\mathcal{O}^{\text {nc }}$ left modules one may use the same formula (13). For bimodules one distinguishes left and right quasicoherence [56, 65, 130] (in the right hand version the tensoring with $\mathcal{O}(W)$ in formula (13) is from the RHS instead). Notice that the tensoring is nothing other than the formula for a perfect localization (i.e. both $Q^{*}$ and $Q_{*}$ are exact, hence the localization is again a ring).

F. VAN OystaEyen ([121]) defines quasicoherent presheaves on the lattice of hereditary torsion theories (= localizations where $Q^{*}$ is exact) over a $\mathbb{Z}_{\geq 0}$-graded rings, by using the appropriate localization instead of tensoring. For affine case see [97, I 6.0.3 (b) and I 6.2. One has theorems on gluing of such modules using Barr-Beck lemma.

D. ORLOV (83]) defines quasicoherent (pre)sheaves on $X$ where $X$ is a presheaf of sets (in particular, by Yoneda, on any object) on a given ringed site $(\mathcal{C}, \mathcal{T})$.

10.4 Now we will quote two theorems. A theorem of Deligne shows that in the context of commutative affine schemes, a formula which can be recognized as a localization at a Gabriel filter (see below), describes the behaviour of the category of quasicoherent sheaves under passage to a not necessarily affine subset. Hence the "noncommutative localization" is already seen there! The theorem may be proved directly, and we suggest to the reader to at least convince oneself that the RHS formula is indeed an $A$-module. "Deligne theorem" ([49]) in fact, cf. ([50], Appendix $)^{1}$, has been originally inferred

\footnotetext{
${ }^{1}$ I thank Prof. HARTSHORNe for the email on the history of the result.
} 
from the Gabriel theorem below - the general statement that quasicompact open subsets of (nonaffine) schemes correspond always to exact localizations of abelian categories; combined with the Gabriel's formulas on how such localizations look like (34).

10.4a Deligne's theorem. Let $X=\operatorname{Spec} A$ be an affine Noetherian scheme, i.e. $A$ is a commutative Noetherian ring. Let $U$ be a Zariski open set (not necessarily affine!), and $I$ an ideal such that $V(I)=X \backslash U$. Let $M$ be an $A$-module, and $\tilde{M}$ the corresponding quasicoherent $\mathcal{O}_{X}$-module. Denote by $\left.\tilde{M}\right|_{U}$ its restriction to $U$. Then

$$
\Gamma\left(\left.\tilde{M}\right|_{U}\right) \cong \lim _{n \rightarrow \infty} \operatorname{Hom}_{A}\left(I^{n}, M\right) .
$$

This isomorphism of $A$-modules is natural in $M$.

10.4b (P. Gabriel 34, VI.3) Let $\left(X, \mathcal{O}_{X}\right)$ be a scheme, and $U$ an open subset of $X$, such that the canonical injection $j: U \hookrightarrow X$ is quasicompact. Let $Q_{U}^{*}: \mathfrak{Q} \mathfrak{c o h} \mathfrak{h}_{X} \rightarrow \mathfrak{Q} \mathfrak{c o h} \mathfrak{h}_{U}$ be a functor which associates to every quasicoherent $\mathcal{O}_{X}$-module $M$ the restriction $\left.M\right|_{U}$ of $M$ to subscheme $\left(U,\left.\mathcal{O}_{X}\right|_{U}\right)$. Functor $Q_{U}^{*}$ canonically decomposes as $\mathfrak{Q} \mathfrak{c o h} \mathfrak{h}_{X} \rightarrow \mathfrak{Q} \mathfrak{c o h}{ }_{X} / \operatorname{Ker} Q_{U}^{*} \cong \mathfrak{Q}_{\mathfrak{c o h}}$ into the canonical projection onto the quotient category by $\operatorname{Ker} Q_{U}^{*}$ and an isomorphism.

\section{Ring maps vs. module functors}

11.1 As usual, we write ${ }_{R} M$ when we want to emphasize that $M$ is (understood as) a left $R$-module; $R$ - Mod is the category of left $R$-modules. Let $f: R \rightarrow S$ be any map of (not necessarily unital) rings. We have the following functors induces by map $f$ :

- extension of scalars $f^{*}: R-\operatorname{Mod} \rightarrow S-\operatorname{Mod}, M \mapsto S \otimes_{R} M$;

- restriction of scalars (forgetful functor) $f_{*}: S-\operatorname{Mod} \rightarrow R-$ Mod, ${ }_{S} M \mapsto{ }_{R} M$;

- $f^{!}: R-\operatorname{Mod} \rightarrow S-\operatorname{Mod}, M \mapsto \operatorname{Hom}_{R}\left({ }_{R} S, M\right)$.

Denote $F \dashv G$ when functor $F$ is left adjoint to functor $G$. Easy fact: $f^{*} \dashv f_{*} \dashv f^{!}$. Hence $f^{*}$ is left exact, $f^{!}$right exact and $f_{*}$ exact.

11.2 It is of uttermost importance to have in mind the geometrical picture of this situation in the case when $R$ and $S$ are commutative and unital. Denote by $\mathfrak{I} \mathfrak{s p}$ the category of locally ringed spaces. An object $\left(X, \mathcal{O}_{X}\right) \in \mathfrak{I} \mathfrak{S p}$ is a pair of a topological space $X$ and a sheaf of commutative local rings $\mathcal{O}_{X}$ over $X$; and a morphism is a pair 
$\left(f^{o}, f^{\sharp}\right)$ where $f^{o}: X \rightarrow Y$ is a map of topological spaces and a 'comorphism' $f^{\sharp}: f^{\bullet} \mathcal{O}_{Y} \rightarrow \mathcal{O}_{X}$ is a map of sheaves of local rings over $X$, and $f^{\bullet}$ a sheaf-theoretic pullback functor. There is a contravariant functor Spec : CommRings $\rightarrow \mathfrak{I S p}$ assigning spectrum to a ring. Map $f: R \rightarrow S$ is replaced by a map of locally ringed spaces

$$
\text { Spec } f=\left(f^{0}, f^{\sharp}\right): \operatorname{Spec} S \rightarrow \operatorname{Spec} R \text {. }
$$

The comorphism $f^{\sharp}$ is important: e.g. if $f^{o}: X \hookrightarrow Y$ is an inclusion, the difference between a subvariety $\left(X, \mathcal{O}_{X}\right)$ and, say, its $n$-th infinitesimal neighborhood in $\left(Y, \mathcal{O}_{Y}\right)$, may be expressed by a proper choice of $f^{\sharp}$.

11.3 After geometrizing rings, one may proceed to geometrize modules. The basic fact here is the affine SERRE's theorem establishing a correspondence $M \leftrightarrow \tilde{M}$ between the $R$-modules and quasicoherent sheaves of $\mathcal{O}_{X}$-modules, for $X=\operatorname{Spec} R$. This correspondence is an equivalence of categories $R-\operatorname{Mod} \leftrightarrow \mathfrak{Q} \mathfrak{c o h} X$. Using this equivalence of categories, functors $f^{*}$ and $f_{*}$ may be rephrased as additive functors

$$
f^{*}: \mathfrak{Q} \mathfrak{c o h} X \rightarrow \mathfrak{Q} \mathfrak{c o h} Y, \quad f_{*}: \mathfrak{Q} \mathfrak{c o h} Y \rightarrow \mathfrak{Q} \mathfrak{c o h} X
$$

and moreover, these can be defined for any morphism $f=\left(f^{o}, f^{\sharp}\right)$ between locally ringed spaces. In this wider context, functor $f^{*}$ is called the inverse image functor of $f$, given by $f^{*} \mathcal{F}:=\mathcal{O}_{Y} \otimes_{\mathcal{O}_{X}} f^{\bullet} \mathcal{F}$ where $f^{\bullet} \mathcal{F}$ is usual, sheaf theoretic, pullback of sheaf $\mathcal{F}$ via $f^{o}$. The restriction of scalars functor then generalizes to the direct image functor for sheaves which is on presheaf level given by

$$
f_{*} \mathcal{F}(U):=\mathcal{F}\left(f^{-1}(U)\right)
$$

and which sends $\mathcal{O}_{X}$-modules to $\mathcal{O}_{Y}$-modules via $\mathcal{O}_{Y}$-action given by the composition of $f^{\sharp} \times$ id and the $\mathcal{O}_{X}$-action. Functors $f^{*}$ and $f_{*}$ are naturally defined between the bigger categories, $\mathcal{O}_{X}-\operatorname{Mod}$ and $\mathcal{O}_{Y}-$ Mod, where they simply preserve the quasicoherence.

11.4 Functor $f_{*}$ (in all settings above) is a right adjoint to $f^{*}$, hence it is left exact and the inverse image functor $f^{*}$ is right exact. This suggests that a pair of adjoint additive functors between abelian categories may be viewed as (coming from) a morphism in geometrical sense. Actually this point of view appears fruitful. On the other hand, surely the choice of a functor in its equivalence class is not essential; 
and the emphasis on the inverse image vs. direct image functor is the matter of choice as well.

Given two abelian categories $\mathcal{A}, \mathcal{B}$, (equivalent to small categories) a morphism $f: \mathcal{B} \rightarrow \mathcal{A}$ is an equivalence class of right exact additive functors from $\mathcal{A}$ to $\mathcal{B}$. An inverse image functor $f^{*}: \mathcal{A} \rightarrow \mathcal{B}$ of $f$ is simply a representative of $f$, which is usually assumed to be made.

An additive functor $f^{*}: \mathcal{B} \rightarrow \mathcal{A}$ between abelian categories is (93])

- continuous if it has a right adjoint, say $f_{*}$;

- flat if it is continuous and exact;

- almost affine if it is continuous and its right adjoint $f_{*}$ is faithful and exact;

- affine if it is almost affine, and its right adjoint $f_{*}$ has its own right adjoint, say $f^{!}$, cf. $\mathbf{1 1 . 1}$.

Morphism $f$ is continuous (flat, almost affine, affine) if its inverse image functor $f^{*}$ is. Some authors say that a functor $F$ is continuous if it has a left adjoint instead, which means that they view $F$ as a direct image functor $f_{*}$ of some continuous morphism $f$. On the other hand, a continuous morphism is coflat if its direct image is exact, and biflat if it is flat and coflat. Usually one equips the categories with distinguished objects ("structure sheaves"); then the morphisms respect the distinguished object.

11.4a Rosenberg (93) introduced an abstract notion of a quasicompact relative noncommutative scheme $(\mathcal{A}, \mathcal{O})$ over a category $\mathcal{C}$ as an abelian category $\mathcal{A}$ with a distinguished object $\mathcal{O}$, finite biflat affine cover by localizations $Q_{\lambda}^{*}: \mathcal{A} \rightarrow \mathcal{B}_{\Lambda}$, with a continuous morphism $g$ from $\mathcal{A}$ to $\mathcal{C}$ (think of it as $X \rightarrow$ Speck) such that each $g_{*} \circ Q_{\lambda *}: \mathcal{B}_{\lambda}$ is affine. This guarantees application of many usual geometric procedure (for basic cohomological needs one does not need $f^{!}$). Such 'schemes' can be related to some spectra and some Grothendieck topologies on $\mathrm{Aff}(\mathrm{Ab})$ ([99]). Quotient spaces for comodule algebras over Hopf algebras may be sometimes constructed as nonaffine noncommutative schemes 104.

11.5 If $R$ and $S$ are rings and ${ }_{S} B_{R}$ a $S-R$-bimodule, then the functor $f^{B}: M \mapsto{ }_{S} B_{R} \otimes_{R} M$ is a right exact functor from $R-\operatorname{Mod}$ to $S$ - Mod. If $S=\mathbb{Z}$ then $B_{R}$ is called flat right $R$-module if $f^{B}$ : $M \mapsto B_{R} \otimes_{R} M$ is exact.

Proposition. (WatTs, 126]) (i) Let $R$ be a (not necessarily unital) ring, and $f^{*}$ a flat endofunctor in $R-\operatorname{Mod}$. Then $f^{*}$ is equivalent to the functor

$$
M \mapsto f^{*}(R) \otimes_{R} M
$$


In particular, $f^{*}(R)$ is flat as a left $R$-module.

(ii) Let $f^{*}: R-\operatorname{Mod} \rightarrow S-\operatorname{Mod}$ be a continuous functor. Then there is a $S-R$-bimodule ${ }_{S} B_{R}$ such that $f^{*}$ is equivalent to the functor $M \mapsto B \otimes_{R} M$.

Notice that when applied to localizations word "flat" is here used in the sense that $Q^{*}$ is flat (weaker), and in some other works means that $Q:=Q_{*} Q^{*}$ is flat. The latter case, for $R-\operatorname{Mod}$, is the situation of Watts theorem, and it is known under the name of perfect localizations. Equivalently ( 87$]$ ), the canonical forgetful functor from the localized category $(R-\mathrm{Mod}) / \mathrm{KerQ}$ to the modules over the localized ring $(Q R)-$ Mod is an equivalence of categories.

11.6 A bicategory (= weak 2 -category) $\mathcal{A}$ consists of

(1) a class $\mathrm{Ob} \mathcal{A}$ of objects ('1-cells');

(2) for each pair of objects $A, B$ a small category $\mathcal{A}(A, B)$; The objects of $\mathcal{A}(A, B)$ are called arrows (or 'morphisms' or '1-cells'), and the morphisms in $\mathcal{A}(A, B)$ are called 2-cells;

(3) for each triple of objects $A, B, C$ a bifunctor ('composition map')

$$
\Phi_{A, B, C}: \mathcal{A}(A, B) \times \mathcal{A}(B, C) \rightarrow \mathcal{A}(A, C),
$$

(4) for each object $A \in \mathcal{A}$, an arrow $1_{A} \in \mathcal{A}(A, A)$ ('identity arrow');

together with natural equivalences

$$
\begin{gathered}
a_{A B C D}: \Phi_{A C D}\left(\Phi_{A B C} \times \mathrm{Id}\right) \Rightarrow \Phi_{A B D}\left(\mathrm{Id} \times \Phi_{B C D}\right), \\
\lambda_{A B C}: \operatorname{Id} \times \Phi_{A B C} \Rightarrow \Phi_{A B C}, \quad \rho_{A B C}: \Phi_{A B C} \times \operatorname{Id} \Rightarrow \Phi_{A B C},
\end{gathered}
$$

satisfying some natural conditions. If $a_{A B C D}, \rho_{A B C}, \lambda_{A B C}$ are all identities, then the bicategory is called (strict) 2-category.

We omit further details in the definition, as our aim is just to sketch a most important example to us: the bicategory of rings and bimodules $\mathfrak{B i m}$. Before that notice that the each monoidal category $\tilde{\mathcal{C}}=\left(\mathcal{C}, \otimes, 1_{\mathcal{C}}, a, l, r\right)$ canonically forms a bicategory $\Sigma \tilde{\mathcal{C}}$ with only one object, being $0:=\mathcal{C}$ itself, and with $\Sigma \tilde{\mathcal{C}}(0,0):=\tilde{\mathcal{C}}$ as far as objects and morphisms are concerned but with the composition $M \circ_{\Sigma \tilde{\mathcal{C}}} N:=M \otimes$ $N$; one further defines $a(M, N, P):=a_{0,0,0}(M, N, P), l_{A}:=\lambda_{0,0}(A)$ and $r_{A}:=\rho_{0,0}(A)$. The definitions may be reversed to form a monoidal category out of any bicategory with a distinguishes object 0 (forgetting about the other objects). Hence we may consider monoidal categories as bicategories with a single object; the notion of being 'strict' in both senses also agrees. 
The objects of $\mathfrak{B i m}$ are unital rings. For any $R, S \in \mathrm{Ob} \mathfrak{B i m}$ define $\mathfrak{B i m}(R, S)$ to be the category of $R$-S-bimodules and $(R, S)$-bilinear mappings between them. The composition

$$
\mathfrak{B i m}(R, S) \times \mathfrak{B i m}(S, T) \rightarrow \mathfrak{B i m}(R, T)
$$

is given by the tensor product functor $(M, N) \mapsto{ }_{R} M_{S} \otimes_{S}{ }_{S} N_{T}$, the rest of the data being obvious. These data indeed define a bicategory.

The invertible 2-cells of $\mathfrak{B i m}$ are called Morita equivalences. It is observed in applications of noncommutative geometry, for example in physics, that Morita invariance is a general feature of natural constructions.

The Watts' theorem identifies bimodules with flat functors. As a pair of adjoint functors, they resemble geometric morphisms among topoi. M. VAN DEN Bergh ([118]) defines a (generalized) bimodule to be any pair of adjoint functors between Grothendieck categories. He also considers right exact functors as so-to-say weak (version of) bimodules. Motivated by duality for coherent sheaves PAPPACENA introduces Frobenius bimodules as those adjoint pairs $F \dashv G$ where $F$ is simultaneously left and right adjoint of $G$. In abstract homotopy theoretic setting, the existence of two-sided adjoints is studied with appropriate (Bousfield-type) localization arguments ([76, 81]).

One of motivations for this (81]) is to extend the GROTHENDIECK duality theory for coherent sheaves on varieties to $\mathcal{D}$-modules. This may be viewed as an example of noncommutative geometry. Namely, the role of the structure sheaf $\mathcal{O}$ is played by the sheaf $\mathcal{D}$ of regular differential operators which is a sheaf of noncommutative $\mathcal{O}$-algebras (cf. [131, 86] for the viewpoint of noncommutative geometry at $\mathcal{D}$ modules). In triangulated categories, the Serre-Grothendieck duality is axiomatized as an existence of so-called SERRE functor (11, 12, 13, 84]), with applications at the borderline between the commutative and noncommutative geometry.

It is a remarkable observation, that the noncommutative geometry via operator algebras, could be also organized around concepts of bicategories $(61])$. Roughly speaking, operator algebras $\left(C^{*}\right.$-algebras; von Neumann algebras respectively) are 0 -cells, appropriate bimodules as 1-cells (Hilbert bimodules; correspondences), and monoidal products as 2-cells (Rieffel interior tensor product of Hilbert bimodules; Connes fusion product). Invertible 1-cells are called Morita equivalences in all these cases. There are also analogues concerning regular bibundles over Lie groupoids, and also analogues in symplectic 
and Poisson geometry. The latter may be viewed as a quasiclassical limit of noncommutative geometry. For Morita equivalence of Poisson manifolds and similar notion of symplectic dual pairs see [62, 101.

\section{Ore localization in filtered rings}

After prerequisites on filtrations, we prove some general lemmas on localizations in filtered rings, mainly easy generalizations of some statements quoted without proof in [68] and in manuscript $[69]^{2}$.

We focus on 'negative' filtrations with the main applications in noncommutative deformation of commutative objects. Such filtrations come from power expansions of algebra operations in a deformation parameter $q-1(2,56,65])$. There is a more refined technique in algebraic analysis, algebraic microlocalization, see [119, 121] and the references in [119]. 'Positive' filtrations involve different techniques than ours. In the study of noncommutative projective algebraic geometry one often has a (negative) filtration related to deformation, as well as a positive grading corresponding to the study of projective schemes. The latter grading may be refined to $\mathbb{Z}^{\times n}$-grading or grading with respect to the weight lattice $P$, as in the study of quantum flag varieties. In any case, if the latter, positive, grading complicates the picture one simply works with homogeneous Ore sets, and we shall ignore this simpler issue.

12.1 Given a (typically abelian) category $\mathcal{C}$, e.g. the category of modules over a ring $\mathbf{k}$, a $\mathbb{Z}$-filtration on an object $M$ in $\mathcal{C}$ is a nested (unbounded) sequence of subobjects $F_{*} M=\left\{\cdots \subset F_{n-1} M \subset F_{n} M \subset\right.$ $\left.F_{n+1} M \cdots \subset M\right\}$. A $\mathbb{Z}$-filtered object in $\mathcal{C}$ an object with a $\mathbb{Z}$-filtration on it. All filtrations in this article are assumed to be exhaustive i.e. the supremum subobject $\sup _{n \in \mathbb{Z}} F_{n} M$ and equals $M$ (e.g. for modules $\left.M=\cup_{n} F_{n} M\right)$.

Let $M=\cup_{n \in \mathbb{Z}} F_{n} M$ be a filtered k-module. The degree $d(m)$ of an element $m \in M$ is the smallest integer $n$, if it exists (otherwise $-\infty)$, such that $m \in F_{n} M$ and $m \notin F_{n-1} M$. Conversely, if $d: M \rightarrow$ $\{-\infty\} \cup \mathbb{Z}$ is subadditive $d(a+b) \leq d(a)+d(b)$, and $d(0)=-\infty$, then $d$ is the degree function of a unique exhaustive filtration on $M$, indeed the one where $a \in F_{n} M$ iff $d(a) \leq n$. An (exhaustive) filtration is separated if $\cap_{n} F_{n} M=0$. Then $d(m)$ is finite for all $m \neq 0$. This will be our assumption from now on.

\footnotetext{
${ }^{2}$ I thank VALERY LunTs for introducing me into this subject and sharing his notes.
} 
12.1a $\mathrm{A} \mathbb{Z}$-filtered $\mathbf{k}$-algebra is a $\mathbf{k}$-algebra $(E, \mu)$ with a filtration $F_{*} E$ on $E$ as a k-module where the multiplication $\mu$ restricted to $F_{n} E \times F_{m} E$ takes values within $F_{n+m} E$, for all $n, m$. This obviously generalizes to algebras in any monoidal category $\tilde{\mathcal{C}}:=\left(\mathcal{C}, \otimes, 1_{\mathcal{C}}\right)$ (notice that the resulting notion is different than if we consider these algebras as auxiliary objects in an abstract category $\mathcal{C}^{\prime}$ of algebras, when 12.1a applies, rather than as algebras in a monoidal category $\tilde{\mathcal{C}})$. For $\mathbf{k}=\mathbb{Z}$ we talk about $\mathbb{Z}$-filtered rings.

Given a filtered $\mathbf{k}$-algebra $\left(F_{*} E, \mu\right)$ a $\mathbb{Z}$-filtered $F_{*} E$-module is a $\mathbb{Z}$-filtered k-module $F_{*} M$ such that $F_{n} E$ acting on $F_{m} M$ takes values within $F_{n+m} M$ for all $n$ and $m$. In particular, $F_{*} E$ is a -filtered module over itself.

Given a filtered k-algebra $E=\cup_{n} F_{n} E$, an associated graded algebra is the $\mathbb{Z}$-graded k-module gr $E=\oplus_{n}(\operatorname{gr} E)_{n}:=\oplus_{n} F_{n} E / F_{n-1} E$ with the multiplication defined as follows. The symbol map ${ }^{-}: E \rightarrow$ gr $E, e \mapsto \bar{e}$, maps $e$ to the class $\bar{e}$ of $e$ in $F_{d(e)} E / F_{d(e)-1} E$. An element $c \in \operatorname{gr} E$ is in the image of the symbol map iff $c$ is homogeneous. For $c=\bar{e}, c^{\prime}=\bar{e}^{\prime}$, the formula $c c^{\prime}:=\bar{e} \bar{e}^{\prime}:=\bar{e} e^{\prime}$ does not depend on the choice of $e, e^{\prime}$. Therefore it defines a binary operation. The additive extension of this operation is the associative multiplication on gr $E$.

It is always true $d\left(e+e^{\prime}\right) \leq \max \left\{d(e), d\left(e^{\prime}\right)\right\}$, with equality if $d(e) \neq d\left(e^{\prime}\right)$. On the other hand, if $d(e)=d\left(e^{\prime}\right)$ then $d\left(e+e^{\prime}\right)$ may be in general anything less or equal $d(e)$, as $e$ and $e^{\prime}$ may cancel in several of the top degrees. Consequently the symbol map is not additive. However...

12.2 ...if gr $E$ is a domain, then $d(a b)=d(a) d(b)$, hence the symbol map $E \rightarrow \operatorname{gr} E$ is multiplicative.

12.3 For any subset $S \subset E$ not containing 0, we can always define a filtration on the set $S \times E$, by formula $d(s, e):=d(e)-d(s)$. If $S$ is left Ore, the localized ring $S^{-1} E$ may be constructed as in 5.6, as certain quotient of $S \times E$. Hence we have a filtration on $S^{-1} E$ as a set with degree function $d\left(s^{-1} e\right)=\inf _{s^{\prime-1} e^{\prime}=s^{-1} e} d\left(s^{\prime}, e^{\prime}\right)$. Recall that $(s, e) \sim\left(s^{\prime}, e^{\prime}\right)$ means $\exists \tilde{s} \in S, \exists \tilde{e} \in E, \tilde{s} s=\tilde{e} s^{\prime}$ and $\tilde{s} e=\tilde{e} e^{\prime}$.

If the degree function is multiplicative, e.g. $E$ is a domain, then

$$
\begin{aligned}
d(s, e) & =d(e)-d(s) \\
& =d(e)-d(\tilde{e})-(d(e)-d(\tilde{s})) \\
& =d(\tilde{s} r)-d(\tilde{s} s) \\
& =d\left(\tilde{e} e^{\prime}\right)-d\left(\tilde{e} s^{\prime}\right) \\
& =d\left(e^{\prime}\right)-d\left(s^{\prime}\right) \\
& =d\left(s^{\prime}, e^{\prime}\right)
\end{aligned}
$$


hence taking the infimum in the expression for $d\left(s^{-1} e\right)$ is superfluous, as all the representatives of $s^{-1} e$ give the same result. Therefore the degree is well-defined by $d\left(s^{-1} e\right):=d(e)-d(s)$.

The symbol image of a set $S \subset E$ is denoted by $\bar{S}$. If $S$ is left Ore in $E$, and gr $E$ is a domain, then $\bar{S}$ is clearly left Ore in gr $E$.

12.4 Lemma. If the symbol map $E \rightarrow \operatorname{gr} E$ is multiplicative, then the induced degree function on $S^{-1} E$ is multiplicative as well.

12.5 Proposition. (68, II 3.2)(i) We have a well-defined map $\theta: \bar{S}^{-1}$ gr $E \rightarrow \operatorname{gr} S^{-1} E$ given by $(\bar{s})^{-1} \bar{e} \mapsto \overline{s^{-1} e}$.

(ii) This map is an isomorphism of graded rings.

Proof. (i) Let $s_{1}, s_{2} \in S$ with $\bar{s}_{1}=\bar{s}_{2}$. By $s_{1}^{-1} s_{2}=1+s_{1}^{-1}\left(s_{2}-s_{1}\right)$ we get $s_{1}^{-1} e=s_{2}^{-1} e+s_{1}^{-1}\left(s_{2}-s_{1}\right) s_{2}^{-1} e$. Using $\mathbf{1 2 . 4}$ and $d\left(s_{2}-s_{1}\right)<$ $d\left(s_{1}\right)$, we see that for each $0 \neq e \in E, s_{1}^{-1}\left(s_{2}-s_{1}\right) s_{2}^{-1} e$ is lower degree than $s_{2}^{-1} e$. Thus $\overline{s_{1}^{-1} e}=\overline{s_{2}^{-1} e} \in \overline{S^{-1} E}$. In the same vein, but easier, we see that $\overline{s^{-1} e}$ does not depend on the choice of $e \in \bar{e}$.

Finally, choose different classes $\bar{t}$ and $\bar{f}$ with $(\bar{t})^{-1} \bar{f}=(\bar{s})^{-1} \bar{e}$. That is $\exists \bar{s}_{*} \in \bar{S}, \exists \bar{e}_{*} \in$ gr $E$ with $\bar{s}_{*} \bar{t}=\bar{e}_{*} \bar{s} \in \bar{S}$ and $\bar{s}_{*} \bar{f}=\bar{e}_{*} \bar{e}$. Then $\overline{s_{*} t}=\overline{e_{*} s}$ and $\overline{s_{*} f}=\overline{e_{*} e}$ for any choice of representatives $e_{*}, s_{*}$ of $\bar{e}_{*}, \bar{s}_{*}$. Hence there are $r_{1}, r_{2}$ of lower degrees than $e_{*} s, e_{*} e$ resp. with $s_{*} t=e_{*} s+r_{1}$ and $s_{*} f=e_{*} e+r_{2}$. Then $t^{-1} f=\left(s_{*} t\right)^{-1} s_{*} f=\left(e_{*} s+\right.$ $\left.r_{1}\right)^{-1}\left(e_{*} e+r_{2}\right)$ which by the above equals $\left(e_{*} s\right)^{-1}\left(e_{*} e\right)=s^{-1} e$ up to elements of lower order, provided that $e_{*} s \in S$. As $e_{*} s+r_{1} \in S$, this is always true if $S$ is saturated, see below. However, the conclusion follows without that assumption. Indeed, by left Ore condition, choose $s^{\sharp}, e^{\sharp}$ with $S \ni s^{\sharp}\left(e_{*} s+r_{1}\right)=e^{\sharp} s$. Then $e^{\sharp} e=e^{\sharp} s s^{-1} e=s^{\sharp} e_{*} e+s^{\sharp} r_{1} s^{-1} e$, where by multiplicativity $s^{\sharp} r_{1} s^{-1} e$ is of lower order. In conclusion, $t^{-1} f=\left[s^{\sharp}\left(e_{*} s+r_{1}\right)\right]^{-1} s^{\sharp}\left(e_{*} e+r_{2}\right)=\left(e^{\sharp} s\right)^{-1} e^{\sharp} e+$ lower order $=$ $s^{-1} e+$ lower order, as required.

(ii) The degree of both sides is $d(e)-d(s)$ hence this map respects grading. The candidate $\overline{s^{-1} e} \rightarrow(\bar{s})^{-1} \bar{e}$ for the inverse is well-defined by more straightforward reasons that $\theta$. Namely, if $\overline{t^{-1} f}=\overline{s^{-1} e}$ then $\exists h$ of lower order with $t^{-1} f=s^{-1} e+h=s^{-1}(e+s h)$. As $\bar{e}=\overline{e+s h}$ it is enough to check the case $h=0$. For some $s_{*} \in S, r_{*} \in r$ we have $s_{*} t=r_{*} s \in S$ and $s_{*} f=r_{*} e$. Then $\bar{s}_{*} \bar{t}=\bar{r}_{*} \bar{s} \in \bar{S}$ and $\bar{s}_{*} \bar{f}=\bar{r}_{*} \bar{e}$, hence $\bar{t}^{-1} \bar{f}=\bar{s}^{-1} \bar{e}$ as required.

12.6 Let $N=\cup_{k \in \mathbb{Z}} F_{k} N$, be a right and $M=\cup_{k \in \mathbb{Z}} F_{k} M$ a left filtered $E$-module, then $N \otimes_{\mathbf{k}} M$ is filtered with respect to the unique degree function additively extending formulas $d\left(n \otimes_{k} m\right)=d(n)+d(m)$. The canonical quotient map $p_{E}: N \otimes_{\mathbf{k}} M \rightarrow N \otimes_{E} M$ induces the filtration $F_{k}\left(N \otimes_{E} M\right):=p_{E}\left(F_{k}\left(N \otimes_{\mathbf{k}} M\right)\right)$. If $N$ is a filtered $E^{\prime}-E$ - 
bimodule, one obtains a filtration of $N \otimes_{E} M$ as a left $E^{\prime}$-module. In particular, given $E=\cup_{k} F_{k} E$, where gr $E$ is a domain, and given a filtered left $E$-module $M=\cup_{k} F_{k} M$, any Ore localization $S^{-1} M=$ $S^{-1} E \otimes_{E} M$ is a filtered left $S^{-1} E$-module with the degree function

$$
d\left(s^{-1} m\right)=d\left(s^{-1} \otimes_{E} m\right)=d\left(s^{-1} 1_{E}\right)+d(m)=d(m)-d(s) .
$$

12.7 Lemma. If the symbol map $E \rightarrow \operatorname{gr} E$ is multiplicative, and $M$ a filtered left E-module, then the degree functions are compatible with action in the sense that $d_{M}(e . m)=d_{E}(e) d_{M}(m)$. Furthermore, for any left Ore set $S \subset E$,

$$
\begin{aligned}
d_{S^{-1} M}\left(s^{-1} e . t^{-1} m\right) & =d_{S^{-1} E}\left(s^{-1} e\right) d_{S^{-1} M}\left(t^{-1} m\right) \\
& =d_{E}(e)+d_{M}(m)-d_{E}(s)-d_{E}(t) .
\end{aligned}
$$

12.8 Proposition. (i) For a filtered ring E, for which gr $E$ is a domain, and any filtered $E$-module $M$, we have a well-defined map $\theta_{M}: \bar{S}^{-1} \operatorname{gr} M \rightarrow \operatorname{gr} S^{-1} M$ given by $(\bar{s})^{-1} \bar{m} \mapsto \overline{s^{-1} m}$.

(ii) $\theta_{M}$ is an isomorphism of graded gr $S^{-1} E=\bar{S}^{-1}$ gr $E$-modules.

The proof is by the same techniques as 12.5. The compatibility with the action $\mathbf{1 2 . 7}$ replaces the multiplicativity, and the formula (9) for the equivalence relation $\sim$ on $S \times M\left(\right.$ with $\left.(S \times M / \sim) \cong S^{-1} M\right)$ replaces the equivalence relation $\sim$ from 4.13 on $S \times R$ in that proof.

12.9 Ore conditions recursively. (i) Let $S$ be a multiplicative set in a ring $E$ with an exhaustive filtration

$F_{*} E=\left\{\ldots \subset F_{-r} E \subset F_{-r+1} E \subset \ldots \subset F_{-1} E \subset F_{0} E \subset F_{1} E \subset \cdots \subset E\right\}$.

Let $S$ satisfy the bounded below filtered-relative left Ore condition in $F_{*} E$ :

$\exists r, \infty>r \geq-n, \forall s \in S, \forall k,-r \leq k \leq n, \forall e \in F_{k} E, \exists s^{\prime} \in S$, $\exists e^{\prime} \in E$ such that $s^{\prime} e-e^{\prime} s \in F_{k-1} E$ if $k>-r$, and $s^{\prime} e-e^{\prime} s=0$ if $k=-r$.

Then $S$ satisfies the left Ore condition for $S$ in $E$.

(ii) Assume that $S$ is bounded filtered left reversible in $F_{*} E$ :

$\exists r<\infty, \forall e_{k} \in F_{k} E$, if $\exists s \in S$ with $e_{k} s \in F_{k-1} E$ then $\exists s^{\prime} \in S$ such that $s^{\prime} e_{k} \in F_{k-1} E$ if $k>-r$, and $s^{\prime} e_{k}=0$ if $k=-r$.

Then $S$ is left reversible in $E$.

Proof. (i) Let $s \in S$ and $e=e_{n} \in F_{n} E$. By induction, we can complete sequences $e_{n}, \ldots, e_{-r}, e_{n}^{\prime}, \ldots, e_{-r}^{\prime}$ (here $e_{k}, e_{k}^{\prime} \in F_{k} E$ ) and $s_{n}^{\prime}, \ldots, s_{-r}^{\prime} \in S$, with $e_{k}^{\prime} s=s_{k}^{\prime} e_{k}-e_{k-1}$ for all $k$ with $e_{-r-1}:=0$. By descending induction on $k$,

$$
\left(e_{k}^{\prime}+s_{k}^{\prime} e_{k+1}^{\prime}+\ldots+s_{k}^{\prime} \cdots s_{n-1}^{\prime} e_{n}^{\prime}\right) s=s_{k}^{\prime} \cdots s_{n}^{\prime} e_{n}-e_{k-1}
$$


for each $k>-r$ and finally

$$
\left(e_{-r}^{\prime}+s_{-r}^{\prime} e_{-r+1}^{\prime}+\ldots+s_{-r}^{\prime} s_{-r+1}^{\prime} \cdots s_{n-1}^{\prime} e_{n}^{\prime}\right) s=s_{-r}^{\prime} \cdots s_{n}^{\prime} e_{n} .
$$

(ii) Suppose $e \in F_{k} E=E$ and $e s=0$ for some $s \in S$. It is sufficient to inductively choose a descending sequence and $s_{k+1}^{\prime}=$ $1, s_{k}^{\prime}, s_{k-1}^{\prime}, \ldots, s_{-r}^{\prime} \in S$, with requirements $s_{j}^{\prime} e \in F_{j-1} E$ for all $j>-r$ and $s_{-r}^{\prime} e=0$. Suppose we have chosen $s_{k}, \ldots, s_{j+1}$. Then $\left(s_{j+1}^{\prime} e\right) s=$ $s_{j+1}^{\prime}(e s)=0$ with $s_{j+1}^{\prime} e \in F_{j} E$, hence by assumption there exist some $\tilde{s} \in S$ such that $\tilde{s} s_{j+1}^{\prime} e \in F_{j-1}$. Set therefore $s_{j}^{\prime}:=\tilde{s} s_{j+1}^{\prime} \in S$.

12.9a Let $F_{*} E$ be an exhaustive filtration of $E$ with $F_{-r} E=0$ for some finite $r$, and $S \subset E$ be a multiplicative set. If the symbol image $\bar{S}$ satisfies the left Ore condition in gr $E$ then clearly the conditions in 12.9 hold. Hence $S$ satisfies left Ore condition in $E$ as well.

12.9b Let $t \in E$ be a regular element $(t E=E t)$ in a ring $E$. Then for each $n>0$ the ideal $t^{n} E$ is 2-sided, hence $E_{n}:=E /\left(t^{n} E\right)$ is a quotient ring in which the element $t$ is nilpotent of order less or equal $n$. Rule $F_{-k} E_{n}=\left(t^{k} E\right) /\left(t^{n} E\right) \subset E /\left(t^{n} E\right) \equiv E_{n}$ defines a bounded 'negative' filtration

$$
F_{*} E_{n}=\left\{0=F_{-n} E \subset \ldots \subset F_{-1} E \subset F_{0} E=E\right\}
$$

in which (the image of) $t$ is of degree -1 . If gr $E$ is a domain then both the symbol map $E \rightarrow \operatorname{gr} E$, and its truncation $E_{n} \rightarrow \operatorname{gr} E_{n}$ are multiplicative.

12.10 Theorem. Let $S$ be a left Ore set in some ring $E_{n}=$ $F_{0} E_{n}$ with a bounded negative filtration $F_{\bullet} E_{n}$. Suppose $S^{\prime} \subset E_{n}$ is a multiplicative set such that $s^{\prime} \in S^{\prime} \cap F_{j} E$ iff $\exists b \in F_{j-1} E$ such that $s^{\prime}=s-b$. Then $S^{\prime}$ is left Ore as well and $S^{-1} E_{n}=\left(S^{\prime}\right)^{-1} E_{n}$ as graded rings.

Proof. As the left Ore localization is a universal object in the category $\mathcal{C}_{l}\left(E_{n}, S\right)$ it is enough to see that a map of rings $j: E_{n} \rightarrow$ $Y$ is in it iff it is in $\mathcal{C}_{l}\left(E_{n}, S^{\prime}\right)$. If $j(s)$ is invertible in $Y$, let $c=$ $j(s)^{-1} j(b)$. Mapping $j$ induces a (non-separated in general) filtration on $Y$ such that $j$ is a map of filtered rings, by taking the degree to be $-\infty$ for elements which are not of the subring $j(S)^{-1} j(R) \subset Y$ and the infimum of expressions $d(e)-d(t)$ for elements which can be represented as $j(t)^{-1} j(e)$. With our numerical constraints on the original degree this difference can not be less than $-n$, if $e \neq 0$. As $d(c)<0$ we obtain $d\left(c^{n}\right)<n-1$, hence $c^{n}=0$. Thus we can invert $j(s)^{-1}(j(s-b))=1-c$ to obtain the geometrical progression $\sum_{j=0}^{n-1} c^{j}$. Then $\sum_{j=0}^{n-1} c^{j} j(s)^{-1} j(s-b)=1$ hence $j(s-b)$ is invertible in $Y$. 
It remains to check also that $s e=0$ for some $s \in S$ iff there exists $s^{\prime \prime} \in S^{\prime}$ with $s^{\prime \prime} e=0$. We proceed by induction on degree $j$ of $e$ starting at $-n$ where $s^{\prime} e-s e \in F_{-n-1}=0$ for $s^{\prime}=s-b$ with the degree of $b$ smaller than of $s^{\prime}$ hence negative. For any $j, s^{\prime} e=(s-b) e=-b e$ has the degree at most $j-1$. On the other hand, by the left Ore condition, we can find $s_{*} \in S$, and $b_{*} \in E$ with $s_{*} b e=b_{*} s e=0$, hence $s_{*}\left(s^{\prime} e\right)=0$. Take $e^{\prime}:=s^{\prime} e$ and notice that $s_{*} e^{\prime}=0$ with the degree of $e^{\prime}$ being less than degree of $e$, hence by the assumption of induction, there exists $s_{*}^{\prime} \in S^{\prime}$ with $s_{*}^{\prime} s^{\prime} e=s_{*}^{\prime} e^{\prime}=0$. Set $s^{\prime \prime}:=s_{*}^{\prime} s^{\prime}$.

The induced grading on the 2 localized rings is the same as the symbol maps at $s$ and at $s^{\prime}=s+b$ evaluates as the same (or, alternatively, they are induced by the same ring map).

Definition. A multiplicative subset $S \subset E_{n}$ is

- admissible if $\forall s \in S, 0 \neq \bar{s} \in$ gr $E_{n}$;

- saturated if $S=\left\{s \in E_{n} \mid \bar{s} \in \bar{S}\right\}$.

12.11 Corollary. Let $E, t, E_{n}, F_{\bullet} E_{n}$ be as in 12.9. Suppose gr $E$ is a commutative domain. Let $S$ be a multiplicative subset in $E_{n}$. Then

a) $S$ is left and right Ore.

b) $S^{-1} E_{n} \neq 0$ iff $S$ is admissible.

c) $S^{-1} E_{n}$ depends only on $\bar{S} \subset \bar{E}$.

d) $S^{-1} E_{n}$ is filtered by powers of $t$ and $\left(S^{-1} E_{n}\right) /\langle t\rangle \cong \bar{S}^{-1} E_{n}$.

e) Any two saturated Ore sets $S, T$ are compatible, i.e. $S^{-1} T^{-1} E_{n} \cong$ $T^{-1} S^{-1} E_{n}$ and $S T=\{s t \mid s \in S, t \in T\}$ is also saturated.

f) Let $S$ be admissible. Then $\operatorname{gr}\left(S^{-1} E_{n}\right) \cong \bar{S}^{-1} \operatorname{gr} E_{n}$. In particular, $\overline{S^{-1} E_{n}} \cong \bar{S}^{-1} \bar{E}_{n}$.

Sketch of the proof. a) follows as a simple case of $\mathbf{1 2 . 9}$; b) is trivial; c) follows by $\mathbf{1 2 . 1 0}$; d) is evident; f) follows from $\mathbf{1 2 . 8}$ after truncating both sides from $E$ to the quotient filtered ring $E_{n}$ (it is not a special case of $\mathbf{1 2 . 8}$, though, as $E_{n}$ is not a domain); e) Because $T$ is saturated, $\left(T^{-1} E\right)_{n} \cong T^{-1} E_{n}$. In $T^{-1} E_{n}$, set $S$ is still multiplicative, hence by a) applied to $\left(T^{-1} E\right)_{n}$ it is left Ore. This is equivalent to compatibility (cf. Sec. 10).

\section{Differential Ore condition}

An extensive literature is dedicated to differential structures of various kind associated to objects of noncommutative geometry: derivations and rings of regular differential operators on $\mathrm{NC}$ rings, $1^{\text {st }}$ and higher 
order differential calculi, with and without (bi)covariance conditions, $\mathrm{NC}$ connections and de Rham complexes etc.

13.1 Let $\partial: R \rightarrow R$ be an $R$-valued derivation on $R$ and $S$ a left Ore set in $R$. Then the formula

$$
\bar{\partial}\left(s^{-1} r\right)=s^{-1} \partial(r)-s^{-1} \partial(s) s^{-1} r, \quad s \in S, r \in R,
$$

defines a derivation $\bar{\partial}: S^{-1} R \rightarrow S^{-1} R$.

The same conclusion if we started with $\partial: R \rightarrow S^{-1} R$ instead.

Proof. 1. $\bar{\partial}$ IS WELL DEFINED.

Suppose $s^{-1} r=t^{-1} r^{\prime}$ for some $r, r^{\prime} \in R, s, t \in S$. Then

$$
\begin{aligned}
\exists \tilde{s} \in S, \exists \tilde{r} \in R, \quad \tilde{s} t=\tilde{r} s, \quad \tilde{s} r^{\prime}=\tilde{r} r & s^{-1}=t^{-1} \tilde{s}^{-1} \tilde{r} \\
& t^{-1} \partial\left(r^{\prime}\right)=t^{-1} \tilde{s}^{-1} \tilde{s} \partial\left(r^{\prime}\right) \\
= & t^{-1} \tilde{s}^{-1}\left[\partial\left(\tilde{s} r^{\prime}\right)-\partial(\tilde{s}) r^{\prime}\right] \\
= & t^{-1} \tilde{s}^{-1}\left[\partial(\tilde{r} r)-\partial(\tilde{s}) r^{\prime}\right] \\
& =t^{-1} \tilde{s}^{-1} \tilde{s} \partial(t) \\
& =t^{-1} \tilde{s}^{-1}[\partial(\tilde{s} t)-\partial(\tilde{s}) t] \\
& =t^{-1} \tilde{s}^{-1}[\partial(\tilde{r} s)-\partial(\tilde{s}) t] \\
\bar{\partial}\left(t^{-1} r^{\prime}\right)= & t^{-1} \partial\left(r^{\prime}\right)-t^{-1} \partial(t) t^{-1} r^{\prime} \\
= & t^{-1} \tilde{s}^{-1}\left[\partial(\tilde{r} r)-\partial(\tilde{s}) r^{\prime}\right]-t^{-1} \tilde{s}^{-1}[\partial(\tilde{r} s)-\partial(\tilde{s}) t] t^{-1} r^{\prime} \\
= & t^{-1} \tilde{s}^{-1} \partial(\tilde{r} r)-t^{-1} \tilde{s}^{-1} \partial(\tilde{r} s) t^{-1} r^{\prime} \\
= & t^{-1} \tilde{s}^{-1} \partial(\tilde{r} r)-t^{-1} \tilde{s}^{-1} \partial(\tilde{r} s) s^{-1} r \\
= & t^{-1} \tilde{s}^{-1} \partial(\tilde{r}) r+t^{-1} \tilde{s}^{-1} \tilde{r} \partial(r) \\
= & -t^{-1} \tilde{s}^{-1} \partial(\tilde{r}) s s^{-1} r-t^{-1} \tilde{s}-1 \tilde{r} \partial(s) s^{-1} r \\
= & \bar{\partial}\left(s^{-1} r\right)
\end{aligned}
$$

2. $\bar{\partial}$ IS A DERIVATION. We have to prove that for all $s, t \in S$ and $r, r^{\prime} \in R$

$$
\bar{\partial}\left(s^{-1} r t^{-1} r^{\prime}\right)=\bar{\partial}\left(s^{-1} r\right) t^{-1} r^{\prime}+s^{-1} r \bar{\partial}\left(t^{-1} r^{\prime}\right) .
$$

The argument of $\bar{\partial}$ on the left hand side has to be first changed into a left fraction form before we can apply the definition of $\bar{\partial}$. By the left Ore condition, we can find $r_{*} \in R, s_{*} \in S$ such that $r_{*} t=s_{*} r$ i.e. $r t^{-1}=s_{*}^{-1} r_{*}$.

We first prove identity (15) in the case $s=r^{\prime}=1$ i.e.

$$
\bar{\partial}\left(r t^{-1}\right)=\partial(r) t^{-1}+r \bar{\partial}\left(t^{-1}\right) .
$$


The left-hand side of (16) is

$$
\begin{aligned}
\bar{\partial}\left(r t^{-1}\right) & =\bar{\partial}\left(s_{*}^{-1} r_{*}\right) \\
& =s_{*}^{-1} \partial\left(r_{*}\right)-s_{*}^{-1} \partial\left(s_{*}\right) s_{*}^{-1} r_{*} \\
& =s_{*}^{-1} \partial\left(r_{*}\right)+\bar{\partial}\left(s_{*}^{-1}\right) r_{*} .
\end{aligned}
$$

The right-hand side of (16) is

$$
\begin{aligned}
\partial(r) t^{-1}-r t^{-1} \partial(t) t^{-1} & =\partial(r) t^{-1}-s_{*}^{-1} r_{*} \partial(t) t^{-1} \\
& =\partial(r) t^{-1}-s_{*}^{-1} \partial\left(r_{*} t\right) t^{-1}-s_{*}^{-1} \partial\left(r_{*}\right) t t^{-1} \\
& =\partial(r) t^{-1}-\bar{\partial}\left(s_{*}^{-1} r_{*} t\right) t^{-1}+\bar{\partial}\left(s_{*}^{-1}\right) r_{*}-s_{*}^{-1} \partial\left(r_{*}\right) \\
& =\partial(r) t^{-1}-\partial(r) t^{-1}-\bar{\partial}\left(s_{*}^{-1}\right) r_{*}-s_{*}^{-1} \partial\left(r_{*}\right) \\
& =\bar{\partial}\left(s_{*}^{-1}\right) r_{*}-s_{*}^{-1} \partial\left(r_{*}\right) .
\end{aligned}
$$

Hence (16) follows. Using (16), we prove (15) directly:

$$
\begin{gathered}
\bar{\partial}\left(s^{-1} r t^{-1} r^{\prime}\right)=\bar{\partial}\left(\left(s_{*} s\right)^{-1} r_{*} r^{\prime}\right) \\
=\left(s_{*} s\right)^{-1} \partial\left(r_{*} r^{\prime}\right)-\left(s_{*} s\right)^{-1} \partial\left(s_{*} s\right)\left(s_{*} s\right)^{-1} r_{*} r^{\prime} \\
=s^{-1} s_{*}^{-1} \partial\left(r_{*}\right) r^{\prime}+s^{-1} s_{*}^{-1} r_{*} \partial\left(r^{\prime}\right) \\
\quad-s^{-1} s_{*}^{-1} \partial\left(s_{*}\right) s_{*}^{-1} r_{*} r^{\prime}-s^{-1} \partial(s) s^{-1} s_{*}^{-1} r_{*} r^{\prime} \\
=s^{-1} s_{*}^{-1} \partial\left(r_{*}\right) r^{\prime}+s^{-1} t^{-1} r \partial\left(r^{\prime}\right)+s^{-1} \bar{\partial}\left(s_{*}^{-1}\right) r_{*} r^{\prime}+\bar{\partial}\left(s^{-1}\right) s_{*}^{-1} r_{*} r^{\prime} \\
=s^{-1} \bar{\partial}\left(s_{*}^{-1} r_{*}\right) r^{\prime}-s^{-1} \bar{\partial}\left(s_{*}^{-1}\right) r_{*} r+s^{-1} r t^{-1} \partial\left(r^{\prime}\right)+\bar{\partial}\left(s^{-1}\right) r t^{-1} r^{\prime} \\
=s^{-1} \bar{\partial}\left(r t^{-1}\right)-s^{-1} \bar{\partial}\left(s_{*}^{-1}\right) r_{*} r+s^{-1} r t^{-1} \partial\left(r^{\prime}\right)+\bar{\partial}\left(s^{-1}\right) r t^{-1} r^{\prime} \\
\underline{\underline{16}} s^{-1} \partial(r) t^{-1} r^{\prime}+s^{-1} r \bar{\partial}\left(t^{-1}\right) r^{\prime}+s^{-1} r t^{-1} \partial\left(r^{\prime}\right)+\bar{\partial}\left(s^{-1}\right) r t^{-1} r^{\prime} \\
\left.=s^{-1} r\right) t^{-1} r^{\prime}+s^{-1} r \bar{\partial}\left(t^{-1} r^{\prime}\right) .
\end{gathered}
$$

Standard textbooks have incomplete proofs of 13.1, e.g. [28, 100].

13.2 Definition. A Poisson bracket on an unital associative k-algebra is an antisymmetric bilinear operation $\{\}:, A \otimes A \rightarrow A$ satisfying the Jacobi identity $\{f,\{g, h\}\}+\{h,\{f, g\}\}+\{g,\{h, f\}\}=0$ for all $f, g, h \in A$ and such that for each $f$, k-linear map $X_{f}: g \mapsto$ $\{f, g\}$ is a k-derivation of $A$. A Poisson algebra is a commutative algebra with a Poisson bracket.

Proposition. Let $A$ be a k-algebra with a Poisson bracket $\{$,$\} ,$ and $S \subset A \backslash\{0\}$ a central multiplicative set. Then

(i) $S^{-1} A$ posses a bilinear bracket $\{\}=,\{,\}^{S}$ such that the localization map $\iota_{S}: A \rightarrow S^{-1} A$ respects intertwines the brackets $\{,\}^{S} \circ$ $\left(\iota_{S} \otimes_{\mathbf{k}} \iota_{S}\right)=\iota_{S} \circ\{$,$\} .$

(ii) If either the Poisson bracket $\{s, t\} \in \operatorname{Ker} \iota_{S}$ whenever $s, t \in S$, or if $A$ is commutative algebra, then there is unique such $\{,\}^{S}$ which is also skew-symmetric. 
(iii) If $A$ is commutative then this unique $\{,\}^{S}$ is a Poisson bracket.

Proof. (i) Each $X_{b}$ by $\mathbf{1 3 . 1}$ induces a unique derivation $X_{b}^{S}=\bar{\partial}$ on $S^{-1} A$ by (14) for $\partial=X_{b}$. Map $b \mapsto X_{b}^{S}$ is k-linear by uniqueness as $X_{b}^{S}+X_{c}^{S}$ is a derivation extending $X_{b+c}$ as well. For each $s^{-1} a \in S^{-1} A$ define k-linear map $Y_{s^{-1} a}: A \rightarrow S^{-1} A$ by

$$
Y_{s^{-1} a}: b \mapsto-X_{b}^{S}\left(s^{-1} a\right)=-s^{-1}\{b, a\}+s^{-1}\{b, s\} s^{-1} a .
$$

Because $s$ is central, $Y_{s^{-1} a}$ is a k-linear derivation. Namely,

$$
\begin{aligned}
Y_{s^{-1} a}(b c)= & -s^{-1}\{b c, a\}+s^{-1}\{b c, s\} s^{-1} \\
= & -s^{-1}\{b, a\} c-s^{-1} b\{c, a\}+ \\
& +s^{-1} b\{c, s\} s^{-1} a+s^{-1}\{b, s\} c s^{-1} a,
\end{aligned}
$$

and, on the other hand,

$$
\begin{aligned}
Y_{s^{-1} a}(b) c+b Y_{s^{-1} a}(c)= & -s^{-1}\{b, a\} c+s^{-1}\{b, s\} s^{-1} a c- \\
& -b s^{-1}\{c, a\}+b s^{-1}\{c, s\} s^{-1} a .
\end{aligned}
$$

Hence $Y_{s^{-1} a}$ extends to a derivation $Y_{s^{-1} a}^{S}$ on $S^{-1} A \rightarrow S^{-1} A$ by formula (14) as well. Define $\left\{s^{-1} a, t^{-1} b\right\}:=Y_{s^{-1} a}^{S}\left(t^{-1} b\right)$.

(ii) To show skew-symmetry we calculate,

$$
\begin{aligned}
Y_{s^{-1} a}^{S}\left(t^{-1} b\right)= & t^{-1} Y_{s^{-1} a}(b)-t^{-1} Y_{s^{-1} a} t^{-1} b \\
= & -t^{-1} X_{b}^{S}\left(s^{-1} a\right)+t^{-1} X_{t}^{S}\left(s^{-1} a\right) t^{-1} b \\
= & -t^{-1} s^{-1} X_{b}(a)+t^{-1} s^{-1} X_{b}(s) s^{-1} a \\
& +t^{-1} s^{-1} X_{t}(a) t^{-1} b-t^{-1} s^{-1} X_{t}(s) s^{-1} a t^{-1} b . \\
Y_{t^{-1} b}^{S}\left(s^{-1} a\right)= & s^{-1} Y_{t^{-1} b}(a)-s^{-1} Y_{t^{-1} b}(s) s^{-1} a \\
= & -s^{-1} X_{a}^{S}\left(t^{-1} b\right)+s^{-1} X_{s}^{S}\left(t^{-1} b\right) s^{-1} a \\
= & -s^{-1} t^{-1} X_{a}(b)+s^{-1} t^{-1} X_{a}(t) t^{-1} b \\
& \quad+s^{-1} t^{-1} X_{s}(b) s^{-1} a-s^{-1} t^{-1} X_{s}(t) t^{-1} b s^{-1} a .
\end{aligned}
$$

Using $X_{b}(a)=-X_{a}(b)$ etc. and centrality of $s, t$ we see that the first 3 terms in $Y_{s^{-1} a}^{S}\left(t^{-1} b\right)$ match with negative sign the first 3 terms (in order 1,3,2) in expression for $Y_{t^{-1} b}^{S}\left(s^{-1} a\right.$ ). If $a$ and $b$ mutually commute, the 4th term agrees the same way, and if they don't but $X_{s}(t)=0$ in the localization $S^{-1} A$, then they are simply 0 , implying skew-symmetry $\left\{s^{-1} a, t^{-1} b\right\}+\left\{t^{-1} b, s^{-1} a\right\}=0$.

Uniqueness: $Z_{s^{-1} a}\left(t^{-1} b\right):=\left\{s^{-1} a, t^{-1} b\right\}$ defines a derivation $Z_{s^{-1} a}$ on $S^{-1} A$, which restricts to a derivation $Z_{s^{-1} a} \mid: A \rightarrow S^{-1} A$. On the 
other hand, $s^{-1} a \mapsto Z_{s^{-1} a}(b)$ is $-X_{b}^{S}$ by its definition. Hence the value of $Z_{s^{-1} a} \mid$ is determined at every $b$, and by $\mathbf{1 3 . 1}$ this fixes $Z_{s^{-1} a}$.

(iii) We'll prove that if the Jacobi rule holds for given $(a, b, c)$ and $(s, b, c)$ in $S^{-1} A^{\times 3}$, then it follows for $\left(s^{-1} a, b, c\right)$ provided $s$ is invertible. By symmetry of the Jacobi rule and by renaming $s^{-1} a \mapsto$ $a$ we infer that it follows for $\left(s^{-1} a, t^{-1} b, c\right)$, as well, and finally for the general case by one more application of this reasoning. Thus we only need to show that $\operatorname{Jacobi}(a, b, c)$ implies $\operatorname{Jacobi}\left(s^{-1} a, b, c\right)$. For commutative $S^{-1} A$ this is a straightforward calculation, using the Jacobi identity, lemma above and skew-symmetry. We name the summands:

$$
\begin{aligned}
\left\{s^{-1} a,\{b, c\}\right\}= & s^{-1}\{a,\{b, c\}\}-s^{-2}\{s,\{b, c\}\} a=:(A 1)+(A 2) \\
\left\{b,\left\{c, s^{-1} a\right\}\right\}= & s^{-1}\{b,\{c, a\}\}-s^{-2}\{b, s\}\{c, a\}-s^{-2}\{b,\{c, s\}\} a \\
& -s^{-2}\{c, s\}\{b, s\} a-s^{-1}\{c, s\}\{b, a\} \\
=: & (B 1)+(B 2)+(B 3)+(B 4)+(B 5) \\
\left\{c,\left\{s^{-1} a, b\right\}\right\}= & s^{-1}\{c,\{a, b\}\}-s^{-2}\{c, s\}\{a, b\}-s^{-2}\{c,\{s, b\}\} a \\
& +s^{-3}\{s, b\}\{c, s\} a-s^{-2}\{s, b\}\{c, a\} \\
=: & (C 1)+(C 2)+(C 3)+(C 4)+(C 5) .
\end{aligned}
$$

Then $(A 1)+(B 1)+(C 1)=0$ and $(A 2)+(B 3)+(C 3)=0$ by Jacobi for $(a, b, c)$, and $(b, c, s)$ respectively. By skew-symmetry $(B 2)+(C 5)=0$, $(B 5)+(C 2)=0$ and $(B 4)+(C 4)=0$ which finishes the proof.

This fact for $A$ (super)commutative is used for example in the theory of (super)integrable systems, sometimes in connection to 'quantization' which is a rich source of examples in noncommutative geometry.

13.3 Let $(R, \cdot,+)$ be a ring (k-algebra), not necessarily unital. A first order differential calculus (FODC) is a $R-R$-bimodule $\Omega^{1}(R)$ together with an additive (k-linear) map $d: R \rightarrow \Omega^{1}(R)$ satisfying Leibnitz identity

$$
d(a b)=d(a) b+a d(b), \quad a, b \in R,
$$

and such that $\Omega^{1}(R)$ is generated by differentials $d r, r \in R$ as a left module. Define a category $\mathfrak{F o d c}$ : objects are pairs of a ring $R$ and a FODC $\left(\Omega^{1}(R), d\right)$ on $R$. A morphisms is a pair $(f, e):\left(R, \Omega^{1}(R), d\right) \rightarrow$ $\left(R^{\prime}, \Omega^{1}\left(R^{\prime}\right), d^{\prime}\right)$ of a ring map $f: R \rightarrow R^{\prime}$ and a map $e: \Omega^{1}(R) \rightarrow$ $\Omega^{1}\left(R^{\prime}\right)$ of $R-R$-bimodules such that $e \circ d=d^{\prime} \circ f$. Fixing $R$ and allowing only morphisms of the form $\left(\operatorname{Id}_{R}, d\right)$ we obtain a (non-full) subcategory $\mathfrak{F} o \mathfrak{d}_{R}$ of $\mathfrak{F o d c}$. If $R$ is unital, then $\left(\operatorname{Ker}\left(R \otimes_{\mathbf{k}} R \rightarrow R, d\right)\right.$ where $d a=1 \otimes a-a \otimes 1$, and the $R$-bimodule structure is ${ }_{R} R \otimes_{\mathbf{k}} R_{R}$, is an initial object of that category. 
Two objects $c_{R}=\left(R, \Omega^{1}(R), d\right), c_{R^{\prime}}=\left(R^{\prime}, \Omega^{1}\left(R^{\prime}\right), d^{\prime}\right)$ in $\mathfrak{F o d c}$ are compatible along $f: R \rightarrow R^{\prime}$ if there is an $e$ such that $(f, e) \in$ $\mathfrak{F o d c}\left(c_{R}, c_{R^{\prime}}\right)$.

Differential calculi restrict: Given $c_{R^{\prime}} \in \mathfrak{F o d c}_{R^{\prime}}$ and $f$ as above, define $f_{\Omega}^{1} \Omega^{1}\left(R^{\prime}\right)$ to be the smallest additive subgroup of $\Omega^{1}\left(R^{\prime}\right)$ containing all the elements of the form $f(a) \partial^{\prime}(f(b)), a, b \in R$. It appears to be an $R-R$-bimodule. Define $f^{\sharp}\left(c_{R^{\prime}}\right):=\left(R, f_{\Omega}^{1} \Omega^{1}\left(R^{\prime}\right), d^{\prime} \circ f\right)$. Then $f^{\sharp}\left(c_{R^{\prime}}\right) \in \mathfrak{F o d c}_{R}$ because $\partial(b) . c=\partial^{\prime}(f(b)) f(c)=\partial^{\prime}(f(b c))-$ $f(b) \partial^{\prime}(f(c))=\partial(b c)-b . \partial(c) \in f^{\sharp} \Omega^{1}\left(R^{\prime}\right)$. where $\partial=\partial^{\prime} \circ f: R \rightarrow$ $f^{\sharp} \Omega^{1}\left(R^{\prime}\right)$ is the restricted differential. Note the decomposition of $(f, e): c_{R} \rightarrow c_{R^{\prime}}$ into $(f, e): c_{R} \rightarrow f^{\sharp} c_{R^{\prime}}$ and $\left(\operatorname{id}_{R^{\prime}}\right.$, incl $): f^{\sharp} c_{R^{\prime}} \rightarrow c_{R^{\prime}}$, where incl : $f_{\Omega^{1}}^{1} \Omega^{1}\left(R^{\prime}\right) \rightarrow \Omega^{1}\left(R^{\prime}\right)$ is the inclusion of $R^{\prime}$-bimodules.

Unlike restricting, there is no general recipe for extending the calculus along ring maps $f: R \rightarrow R^{\prime}$, except for the special case when $R^{\prime}=S^{-1} R$ and $\Omega^{1} R={ }_{R} R_{R}$, treated in 13.1. That case is of central importance in study of the regular differential operators and Dmodules over noncommutative spaces [66, 67, 68]. We'll just mention a slight generalization.

13.4 Theorem. Let $S \subset R$ be a left Ore set in $R$ and suppose $\left\{x \in \Omega^{1}(R) \mid \exists t \in S, x t=0\right\}=0$.

The following are then equivalent:

(i) The $S^{-1} R$-R-bimodule structure on $S^{-1} \Omega^{1}(R) \equiv S^{-1} R \otimes_{R}$ $\Omega^{1}(R)$ extends to an (actually unique) $S^{-1} R$-bimodule structure which may carry a differential $d_{S}: S^{-1} R \rightarrow S^{-1} \Omega^{1}(R)$ such that the pair of localization maps $\left(\iota_{S}, \iota_{S, \Omega^{1}(R)}\right)$ is a morphism in $\mathfrak{F o d c}$ (i.e. 'the calculi are compatible along the localization').

(ii) The differential Ore condition is satisfied:

$$
\forall t \in S, \forall r \in R, \exists s \in S, \exists \omega \in \Omega^{1}(R), \quad s d r=\omega t .
$$

Proof. (i) $\Rightarrow$ (ii). If $S^{-1} \Omega^{1}(R) \equiv S^{-1} R \otimes_{R} \Omega^{1}(R)$ is a $S^{-1} R$-bimodule then $(d r) t^{-1} \in S^{-1} \Omega^{1}(R)$ for $t \in S, r \in R$. All the elements in $S^{-1} \Omega^{1}(R)$ are of the form $s^{-1} \omega$ where $s \in S$ and $\Omega \in \Omega^{1}(R)$. Hence $\exists s \in S, \exists \omega \in \Omega^{1}(R)$ such that $s d r=\omega t$ in the localization. By $\mathbf{7 . 4}$ this means $s d r=\omega t+\omega^{\prime}$ in $\Omega^{1}(R)$, where $s^{\prime} \omega^{\prime}=0$ for some $s^{\prime} \in S$. Pre-multiplying by $s^{\prime}$ we obtain $\left(s^{\prime} s\right) d r=\left(s^{\prime} \omega\right) t$, with required form.

(ii) $\Rightarrow$ (i). The right $S^{-1} R$ action if it exists is clearly forced by

$$
s_{1}^{-1} a d(r) t^{-1} b=s_{1}^{-1} a s^{-1} \omega b
$$

for $s, \omega$ chosen as above. On the other hand, if (17) holds, this right action does extend the right $R$-action. One has to prove that (17) can 
be taken as a definition of right $S^{-1} R$-action (compatible with the left action), i.e. it does not depend on choices. If we choose $s^{\prime}, \omega^{\prime}$ such that $s^{\prime} d(r)=\omega^{\prime} t$ then $s^{-1} \omega t=\left(s^{\prime}\right)^{-1} \omega^{\prime} t$. As $t$ does not annihilate from the right, $s^{-1} \omega=\left(s^{\prime}\right)^{-1} \omega^{\prime}$. Other cases are left to the reader. Hence $S^{-1} \Omega^{1}(R)$ is a bimodule; its elements are of the form $s^{-1} a d b$.

To prove that it is sufficient, define $d_{S}$ from $d$ by the generalization of formula (14) by $\bar{\partial}$ and $\partial$ replaced by $d_{S}$ and $d$ and proceed with the rest of the proof as in $\mathbf{1 3 . 1}$ - all the calculations there make sense.

\section{Gabriel filter $\mathcal{L}_{S}$ for any $S \subset R$}

14.1 A lattice is a poset $(W, \succ)$ such that for any two elements $z_{1}, z_{2}$ the least upper bound $z_{1} \vee z_{2}$ and the greatest lower bound $z_{1} \wedge z_{2}$ exist. In other words, the binary operations meet $\wedge$ and join $\vee$ are everywhere defined. A poset is bounded if it contains a maximum and a minimum element, which we denote 1 and 0 respectively. A ('proper') filter in a bounded lattice $(W, \succ)$ is a subset $\mathcal{L} \subset W$ such that $1 \in \mathcal{L}, 0 \notin \mathcal{L},\left(z_{1}, z_{2} \in \mathcal{L} \Rightarrow z_{1} \wedge z_{2} \in \mathcal{L}\right)$ and $\left(z \in \mathcal{L}, z^{\prime} \succ z \Rightarrow\right.$ $\left.z^{\prime} \in \mathcal{L}\right)$.

E.g. in any bounded lattice $(W, \succ)$, given $m \in W$, the set ${ }^{m} W$ of all $n \succ m$ is a filter.

14.2 Notation. Given a left ideal $J \in I_{l} R$ and a subset $w \subset R$ define

$$
(J: w):=\{z \in R \mid z w \subset J\}
$$

Then $(J: w)$ is a left ideal in $R$. If $w=: K$ is also a left ideal, then $(J: K)$ is 2-sided ideal. In particular, if $w=K=R$, then $(J: R)$ is the maximal 2-sided ideal contained in $J$. For $r \in R$ we write $(J: r)$ for $(J,\{r\})$.

Given subsets $v, w \subset R$, set $((J: v): w)$ contains precisely all $t_{1}$ such that $t_{1} w \subset(J: v)$, i.e. $t_{1} w v \subset J$. Hence $((J: v): w)=(J: w v)$.

14.3 Preorders on left ideals. Let $I_{l} R$ be the set of all left ideals in a ring $R$. It is naturally a preorder category with respect to the inclusion preorder. This category is a lattice. For the localization questions another partial order $\succ$ on $I_{l} R$ is sometimes better: $K \succ J$ (category notation: $J \rightarrow K$ ) iff either $J \subset K$, or there exist a finite subset $w \subset R$ such that $(J: w) \subset K$. Any filter in $\left(I_{l} R, \succ\right)$ is called a uniform filter . 
14.4 Let $R$ be a unital ring and $S \subset R$ a multiplicative set. Consider

$$
\mathcal{L}_{S}:=\{J \text { left ideal in } R \mid \forall r,(J: r) \cap S \neq \emptyset\} \subset I_{l} R .
$$

We make the following observations:

- As $(R: r)=R, R \in \mathcal{L}_{S}$.

- Suppose $J, K \in \mathcal{L}_{S}$. Given $r \in R, \exists s, t$, such that $s \in(J: r) \cap S$ and $t \in(K: s r) \cap S$. Hence $t s r \in J \cap K$. Set $S$ is multiplicative, hence $t s \in S$ and $t s \in(J: r) \cap(K: r) \cap S=(J \cap K: r) \cap S$. Thus $J \cap K \in \mathcal{L}$.

- $(J: r) \cap S \neq \emptyset$ then, a fortiori, $(K: r) \cap S \neq \emptyset$ for $K \supset J$.

- If $J \in \mathcal{L}_{S}$ then $\forall r \quad(J: r) \cap S \neq \emptyset$. In particular, this holds with $r$ replaced by $r r^{\prime}$. Using $\left((J: r): r^{\prime}\right)=\left(J: r^{\prime} r\right)$ we see that $(J: r) \in \mathcal{L}_{S}$ for all $r \in R$.

- If $\forall r^{\prime} \in R\left(J: r^{\prime}\right) \cap S \neq \emptyset$ and $\left(\left(J^{\prime}: j\right): r\right) \cap S \neq \emptyset$ for all $j \in J$, $r \in R$, then $\exists s \in S$ such that $\operatorname{sr} j \in J^{\prime}$ and $\exists s^{\prime} \in S$ such that $s^{\prime} r^{\prime} \in J$. In particular for $r=1$ and $j=s^{\prime} r^{\prime}$ we have $s s^{\prime} r^{\prime} \in J$. Now $s s^{\prime} \in S$ and $r^{\prime}$ is arbitrary so $J^{\prime} \in \mathcal{F}_{S}$.

These properties can be restated as axioms for a Gabriel filter $\mathcal{L} \subset$ $I_{l} R$ (synonyms "radical set", "radical filter", "idempotent topologizing filter"):

- (F1) $R \in \mathcal{L}$ and $\emptyset \notin \mathcal{L}$.

- (F2) If $J, K \in \mathcal{L}$, then $J \cap K \in \mathcal{L}$.

- (F3) If $J \in \mathcal{L}$ and $J \subset K$ then $K \in \mathcal{L}$.

- (UF) $J \in \mathcal{L} \Leftrightarrow(\forall r \in R,(J: r) \in \mathcal{L})$.

- (GF) If $J \in \mathcal{L}$ and $\forall j \in J$ the left ideal $\left(J^{\prime}: j\right) \in \mathcal{L}$, then $J^{\prime} \in \mathcal{L}_{S}$.

(F1-3) are simply axioms saying that a set $\mathcal{L}$ of ideals in $R$ is a filter in $\left(I_{l} R, \subset\right)$, and if we add (UF) we get a notion of a uniform filter (i.e. a filter in $\left(I_{l} R, \succ\right)$.

Even for commutative $R$, there are examples of Gabriel filters $\mathcal{L}$, which are not of the form $\mathcal{L}_{S}$ for a multiplicative $S \subset R$. Moreover, for rings without unity (F1-3,UF,GF) still make sense, whence a good notion of a multiplicative set and filters $\mathcal{L}_{S}$ fails to exist.

Notice that if a multiplicative set $S$ satisfies the left Ore condition, then $\mathcal{L}_{S}=\mathcal{L}_{S}^{\prime}:=\{J$ is left ideal $\mid J \cap S \neq \emptyset\}$. Namely, $(J: 1) \cap S=$ 
$J \cap S$ for any $\mathrm{S}$, hence $\mathcal{L}_{S} \subset \mathcal{L}_{S}^{\prime}$; and the left Ore condition implies that given an element $s \in J \cap S$ and $r \in R$ we can find $s^{\prime} \in S$, and $r^{\prime} \in R$ with $s^{\prime} r=r^{\prime} s \in r^{\prime} J \subset J$, hence $s^{\prime} \in(J: r) \cap S$; hence $\mathcal{L}_{S}^{\prime} \subset \mathcal{L}_{S}$.

14.5 It is simple to check that the intersection of any family of Gabriel filter is a Gabriel filter. However this is not always true for the union: (GF) often fails.

14.6 Proposition. Let $\mathcal{L}$ be a Gabriel filter. (i) Then

$$
\sigma_{\mathcal{L}}(M):=\{m \in M \mid \exists J \in \mathcal{L}, J m=0\} .
$$

is an $R$-submodule of $M$.

(ii) $\sigma_{\mathcal{L}}^{\prime}(M):=\lim _{J \in(\mathcal{L}, \succ)} \operatorname{Hom}_{R}(R / J, M)$ is naturally a left $R$ module.

(iii) If $1 \in R$ then the endofunctors $\sigma_{\mathcal{L}}$ and $\sigma_{\mathcal{L}}^{\prime}$ (on the categories of modules $M$ with $1_{R} m=m$, where $m \in M$ ) are equivalent.

Proof. (i) It is obvious that $\sigma_{\mathcal{L}}(M)$ is closed with respect to the addition. Suppose $m \in \sigma_{\mathcal{L}}(M)$, i.e. $J m=0$ for some $J \in \mathcal{L}$. Let $r \in R$ be arbitrary. As $\mathcal{L}$ is Gabriel filter, one has $K:=(J: r) \in \mathcal{L}$. Let $k \in K$. Then $k r \in J$, hence $k r m=0$. This is true for any such $k$, hence $K r m=0$ and $r m \in \sigma_{\mathcal{L}}(M)$. As $r$ was arbitrary, we have $R \sigma_{\mathcal{L}}(M) \subset \sigma_{\mathcal{L}}(M)$.

(ii) Let $r \in R$ and $f \in \lim \operatorname{Hom}_{R}(R / J, M)$, i.e. $f=\left[f_{J}\right] \in$ $\operatorname{Hom}_{R}(R / J, M)$ for some $J \in(\mathcal{L}, \succ)$. Let $(r f)_{J} \in \operatorname{Hom}_{R}(R /(J$ : $r), M)$ be given by $(r f)_{J}\left(r^{\prime}+(J: r)\right)=f_{J}\left(r^{\prime} r+J\right)$. This is well defined as changing $r^{\prime}$ by an element $\delta r^{\prime} \in(J: r)$ changes $r^{\prime} r$ by an element $\left(\delta r^{\prime}\right) r$ in $(J: r) r \subset J$.

Define $r f$ to be the limit class of $(r f)_{J}$. It is well defined as clearly $f_{J} \sim f_{J^{\prime}}$ implies $(r f)_{J} \sim(r f)_{J^{\prime}}$ by the defining formula for $(r f)_{J}$.

Finally, $f \mapsto r f$ is a left $R$-action. Indeed, for all $r, r^{\prime}, t \in R$,

$$
\begin{aligned}
\left(\left(r r^{\prime}\right) f_{J}\right)\left(t+\left(J: r r^{\prime}\right)\right) & =\left(r r^{\prime}\right) f_{J}\left(t+\left(\left(J: r^{\prime}\right): r\right)\right) \\
& =f_{J}\left(t r^{\prime} r+\left(\left(J: r^{\prime}\right): r\right)\right) \\
& =f_{J}\left(\left(t r^{\prime}\right) r+\left(\left(J: r^{\prime}\right): r\right)\right) \\
& =(r f)_{J}\left(\left(t r^{\prime}\right) r+\left(\left(J: r^{\prime}\right): r\right)\right) \\
& =(r f)_{\left(J: r^{\prime}\right)}\left(t r^{\prime}+\left(\left(J: r^{\prime}\right): r\right)\right) \\
& =\left(r^{\prime}\left[(r f)_{\left(J: r^{\prime}\right)}\right]\right)\left(t+\left(\left(J: r^{\prime}\right): r\right)\right) .
\end{aligned}
$$

If $R$ and $M$ are unital, then $1_{R} f=f$ as well.

(iii) To make the statement precise, we should first extend $\sigma_{\mathcal{L}}$ to a functor by defining it on morphisms as well $\left(\sigma_{\mathcal{L}}^{\prime}\right.$ is obviously a functor as the formula on object is explicitly written in terms of a composition 
of functors applied on $M)$. As $\sigma_{\mathcal{L}}(M) \subset M$, it is sufficient to show that $f\left(\sigma_{\mathcal{L}}(M)\right)=\sigma_{\mathcal{L}}(f(M))$ and then define $\sigma_{\mathcal{L}}(f):=\sigma_{\mathcal{L}} \circ f$. Element $f(m) \in f\left(\sigma_{\mathcal{L}}(M)\right)$ iff $J m=0$ for some $J \in \mathcal{L}$. This is satisfied iff $f(J m)=J f(m)=0$, i.e. $f(m) \in \sigma_{\mathcal{L}}(f(M))$.

The equivalence $\nu: \sigma_{\mathcal{L}}^{\prime} \rightarrow \sigma_{\mathcal{L}}$ is given by $\nu_{M}\left(\left[f_{J}\right]\right):=f_{J}\left(1_{R}+\right.$ $J) \in \sigma_{\mathcal{L}}(M)$ (because $J f_{J}\left(1_{R}+J\right)=f_{J}(0+J)=0$ ), with inverse $m \mapsto\left[f_{J}^{(m)}\right]$ where $f_{J}^{(m)}: r+J \mapsto r m$ and $J m=0$. Starting with $\left[f_{J}\right]$ with $m:=f_{J}\left(1_{R}+J\right)$ and $f_{J}^{(m)}: r+J \mapsto(r f)_{J}\left(1_{R}+J\right)=f_{J}(r+J)$, hence $f_{J}^{(m)}=f_{J}$. Other way around, start with $m \in \sigma_{\mathcal{L}}(M)$, then $\nu_{M}\left(f_{J}^{(m)}\right)=f_{J}^{(m)}\left(1_{R}+J\right)=1_{R} m=m$. Hence we see that each $\nu_{M}$ is an isomorphism of modules. We leave the check that $\nu, \nu^{-1}$ are natural transformations to the reader.

14.7 If $\mathcal{A}$ is any abelian category, then a subfunctor $\sigma$ of the identity (i.e. $\sigma(M) \subset M$ and $\sigma(f)(\sigma(M))=f(\sigma(M))$, cf. 1.4) with the property $\sigma(M / \sigma(M))=0$ is called a preradical in $\mathcal{A}$. A radical is a left exact preradical.

14.8 Proposition. $\sigma_{\mathcal{L}}$ is an idempotent radical in the category of left $R$-modules i.e. it is a radical and $\sigma_{\mathcal{L}} \sigma_{\mathcal{L}}=\sigma_{\mathcal{L}}$.

Proof. $m+\sigma_{\mathcal{L}} M \in \sigma_{\mathcal{L}}\left(M / \sigma_{\mathcal{L}} M\right) \Rightarrow \exists J \in \mathcal{L}, J m \subset \sigma_{\mathcal{L}} M$.

14.9 Let $\mathcal{L}$ be a Gabriel filter. Then for any left $R$-module $M$ the abelian group

$$
H_{\mathcal{L}}(M)=\lim _{J \in \mathcal{L}} \operatorname{Hom}_{R}(J, M)
$$

has a natural structure of an $R$-module. Given $f \in H_{\mathcal{L}}(M)$, we represent it as $f_{J}$ in $\operatorname{Hom}_{R}(J, M)$. For $r \in R$, the left ideal $(J: r) \in \mathcal{L}$, by the definition of a uniform filter, and the rule $x \mapsto f(x r)$ defines an element $(r f)_{(J: r)}$ in $\operatorname{Hom}_{R}\left((J: r), M / \sigma_{\mathcal{L}}(M)\right)$ representing the class of $r f$. This yields a well defined left action.

14.10 To any Gabriel filter $\mathcal{L}$, one associates a localization endofunctor $Q_{\mathcal{L}}$ on the category of left modules by the formula

$$
Q_{\mathcal{L}}(M):=H_{\mathcal{L}}\left(M / \sigma_{\mathcal{L}}(M)\right)=\lim _{J \in \mathcal{L}} \operatorname{Hom}_{R}\left(J, M / \sigma_{\mathcal{L}}(M)\right) .
$$

Left multiplication by an element $r \in R$ defines a class $[r] \in Q_{\mathcal{L}}(R)$. There is a unique ring structure on $Q_{\mathcal{L}}(R)$, such that the correspondence $i_{\mathcal{L}}: r \mapsto[r]$ becomes a ring homomorphism $i_{\mathcal{L}}: R \rightarrow Q_{\mathcal{L}}(R)$.

Notice that (19) is a generalization of RHS of Deligne's formula, 10.4a.

14.11 Not only every Gabriel filter defines an idempotent radical, but also $(\underline{52})$ : 
Proposition. Every radical defines a Gabriel filter by the rule

$$
\mathcal{L}_{\sigma}:=\{J \text { left ideal in } R \mid \sigma(R / J)=R / J\} .
$$

More generally, if $M$ be a left $R$-module and $\sigma$ a radical, define

$$
\mathcal{L}_{M, \sigma}:=\{L \text { left } R \text {-submodule in } M \mid \sigma(M / L)=M / L\} .
$$

Then $\mathcal{L}_{M}:=\mathcal{L}_{M, \sigma}$ satisfies the following properties

- (GT1) $M \in \mathcal{L}_{M}$.

- (GT2) If $L, K \in \mathcal{L}_{M}$, then $L \cap K \in \mathcal{L}$.

- (GT3) If $L \in \mathcal{L}_{M}, K \subset M$ a left ideal and $L \subset K$, then $K \in \mathcal{L}_{M}$.

- (GT4) If $J \in \mathcal{L}_{M}$ and $K \in \mathcal{L}_{J}$ the left ideal $K \in \mathcal{L}_{M}$.

14.11a When we restrict to the idempotent radicals, then the rule $\sigma \mapsto \mathcal{L}_{\sigma}$ gives a bijection between the idempotent radicals and Gabriel filters.

\section{Localization in abelian categories}

The language of Gabriel filters is not suited for some other categories where additive localization functors are useful. Special subcategories closed with respect to useful operations (e.g. extensions of objects) is a wider method, particularly in abelian and triangulated setting. We confine ourselves just to a summary of basic notions in abelian setting and comment on the connection to the language of Gabriel filters, as a number of references is available [17, 34, 35, 52, 87, 91].

15.1 Let $\mathcal{A}$ be an additive category. Let $\mathcal{P}$ be a full subcategory of $\mathcal{A}$. Define the left and right orthogonal to $\mathcal{P}$ to be the full subcategories ${ }^{\perp} \mathcal{P}$ and $\mathcal{P}^{\perp}$ consisting of all objects $A \in \mathcal{A}$ such that $\mathcal{A}(P, A)=0$ (resp. $\mathcal{A}(A, P)=0$ ) for all $P \in \mathcal{P}$. Zero object is the only object in $\mathcal{P} \cap^{\perp} \mathcal{P}$. It is clear that taking (left or right) orthogonal reverses inclusions and that $\mathcal{P} \subset{ }^{\perp}\left(\mathcal{P}^{\perp}\right)$ and $\mathcal{P} \subset\left({ }^{\perp} \mathcal{P}\right)^{\perp}$. We leave as an exercise that $\mathcal{P}^{\perp}=\left({ }^{\perp}\left(\mathcal{P}^{\perp}\right)\right)^{\perp}$ and ${ }^{\perp} \mathcal{P}={ }^{\perp}\left(\left({ }^{\perp} \mathcal{P}\right)^{\perp}\right)$.

15.2 A thick subcategory of an abelian category $\mathcal{A}$ is a replete (= full and closed under isomorphisms) subcategory $\mathcal{T}$ of $\mathcal{A}$ which is closed under extensions, subobjects and quotients. In other words, an object $M^{\prime}$ in a short sequence $0 \rightarrow M \rightarrow M^{\prime} \rightarrow M^{\prime \prime} \rightarrow 0$ in $\mathcal{A}$ belongs to $\mathcal{T}$ iff $M$ and $M^{\prime \prime}$ do. 
Given a pair $(\mathcal{A}, \mathcal{T})$ where $\mathcal{A}$ is abelian and $\mathcal{T} \subset \mathcal{A}$ is thick, consider the class

$$
\Sigma(\mathcal{T}):=\{f \mid \operatorname{Ker} f \in \mathrm{Ob} \mathcal{T}, \text { and Coker } f \in \mathrm{Ob} \mathcal{T}\}
$$

The quotient category $\mathcal{A} / \mathcal{T}$ is defined as follows. $\mathrm{Ob} \mathcal{A} / \mathcal{T}=\mathrm{Ob} \mathcal{A}$ and Mor $\mathcal{A} / \mathcal{T}:=\operatorname{Mor} \mathcal{A} \amalg \Sigma^{-1}(\mathcal{T})$, where $\Sigma^{-1}(\mathcal{T})$ is the class of formal inverses of morphisms $f \in \Sigma$; impose the obvious relations. $\mathcal{A} / \mathcal{T}$ is additive in a unique way making the quotient functor additive. In fact (34, 35]), it is abelian.

Proposition. (GRoTHENDIECK [48]) Let $\mathcal{T}$ be a thick subcategory in $\mathcal{A}$ and $\Sigma(\mathcal{T})$ as above. Then $\Sigma$ is a left and right calculus of fractions in $\mathcal{A}$ and $\mathcal{A}[\Sigma(\mathcal{T})]^{-1}$ is naturally isomorphic to $\mathcal{A} / \mathcal{T}$.

A thick subcategory $\mathcal{T}$ is a localizing subcategory if the morphisms which are invertible in the quotient category $\mathcal{A} / \mathcal{T}$ are precisely the images of the morphisms in $\Sigma(\mathcal{T})$.

Every exact localization functor $T^{*}: \mathcal{A} \rightarrow \mathcal{B}$ (i.e. an exact functor with fully faithful right adjoint $T_{*}$ ) of an abelian category $\mathcal{A}$ is the localization at the localizing subcategory $\Sigma$ consisting of those morphisms $f$ such that $T^{*} f$ is either kernel or a cokernel morphism of an invertible morphism in $\mathcal{B}$.

If $T^{*}: \mathcal{A} \rightarrow \mathcal{B}$ is any exact localization functor, then set $\mathcal{T}:=$ Ker $T^{*}$ to be the full subcategory of $\mathcal{A}$ generated by all objects $X$ such that $T^{*}(X)=0$. Then $T^{*}$ factors uniquely as $\mathcal{A} \stackrel{\mathcal{Q}^{*}}{\rightarrow} \mathcal{A} / \mathcal{T} \rightarrow \mathcal{B}$ where $\mathcal{Q}^{*}$ is the natural quotient map.

More than one thick subcategory may give the same quotient category, and that ambiguity is removed if we consider the corresponding localizing subcategories instead.

A composition of localization functors corresponds to Gabriel multiplication - on thick subcategories. Actually, for any subcategories $\mathcal{B}, \mathcal{D}$ an abelian category $\mathcal{A}$ one defines $D \bullet B$ to be the full subcategory of $\mathcal{A}$ consisting of precisely those $A$ in $\mathcal{A}$ for which there is an exact sequence $0 \rightarrow B \rightarrow A \rightarrow D \rightarrow 0$ with $B$ in $\mathrm{Ob} \mathcal{B}$ and $D$ in $\mathrm{Ob} \mathcal{D}$. There is also a version in terms of radical filters, cf. (97]).

15.3 In this article, we often viewed exact localizations (and quotient categories, cf. 10.4b) as categorical analogues of open spaces. Their complements should then be the complementary data to the quotient categories, and such data are localizing subcategories. More precise and detailed discussion of subcategories which may be considered as subschemes and closed subschemes may be found in [66], Part I and 97, 108]. Cf the notion of a (co)reflective subcategory in 8.9. 
Thus we view that it is more geometrically appealing to split the data of a category to a localizing subcategory and a quotient subcategory, then into two different subcategories. However, the latter point of view is more traditional, under the name of "torsion theory' and has geometrically important analogues for triangulated categories. A torsion theory [17, 52] in Abelian category $\mathcal{A}$ is a pair $(\mathcal{T}, \mathcal{F})$ of replete subcategories of $\mathcal{A}$ such that $\mathcal{F}^{\perp}=\mathcal{T}$ and ${ }^{\perp} \mathcal{T}=\mathcal{F}$.

For any idempotent radical $\sigma$ in $\mathcal{A}$ (14.8), the class $\mathcal{T}_{\sigma}$ of $\sigma$-torsion objects and the class $\mathcal{F}_{\sigma}$ of $\sigma$-torsion free objects are defined by formulas

$$
\mathcal{T}_{\sigma}=\{M \in \mathrm{Ob} \mathcal{A} \mid \sigma(M)=M\}, \quad \mathcal{F}_{\sigma}=\{M \in \mathrm{Ob} \mathcal{A} \mid \sigma(M)=0\} .
$$

This pair $\left(\mathcal{T}_{\sigma}, \mathcal{F}_{\sigma}\right)$ is an example of a torsion theory and $\mathcal{T}_{\sigma}$ is a thick subcategory of $\mathcal{A}$. Not every torsion theory corresponds to a radical, but hereditary theories do. That means that a subobject of a torsion object is torsion. Cohn localization of the last section is not necessarily hereditary, but it is always a torsion theory as shown there.

\section{Quasideterminants and Cohn local- ization.}

Notation. Let $M_{m}^{n}(R)$ be the set of all $n \times m$ matrices over a (noncommutative) ring $R$, so that $M_{n}(R):=M_{n}^{n}(R)$ is a ring as well. Let $I, J$ be the ordered tuples of row and column labels of $A=\left(a_{j}^{i}\right) \in M_{m}^{n}(R)$ respectively. For subtuples $I^{\prime} \subset I, J^{\prime} \subset J$ and $A=\left(a_{j}^{i}\right) \in M_{m}^{n}(R)$, denote by $A_{J^{\prime}}^{I^{\prime}}$ the submatrix of $A_{J}^{I}:=A$ consisting only of the rows and columns with included labels; e.g. $A_{\{j\}}^{\{i\}}=a_{j}^{i}$ is the entry in $i$-th row and $j$-th column. When $I$ is known and $K \subset I$, then $|K|$ is the cardinality of $K$ and ${ }^{\wedge}$ is the symbol for omitting, i.e. $\hat{K}=I \backslash K$ is the complementary $(|K|-|I|)$-tuple.

We may consider the $r$-tuple $\tilde{I}=\left(I_{1}, \ldots, I_{r}\right)$ of sub-tuples which partitions the $n$-tuple $I=\left(i_{1}, i_{2}, \ldots, i_{n}\right)$, i.e. $I_{k}$ are disjoint and all labels from $I$ are included; then $|\tilde{I}|:=r$. Given $\tilde{I}, \tilde{J}$ form the corresponding block matrix in $M_{\tilde{J}}^{\tilde{I}}$ out of $A$, i.e. the $|\tilde{I}| \times|\tilde{J}|$ matrix $A_{\tilde{J}}^{\tilde{I}}$ whose entries are matrices $A_{\tilde{J}_{l}}^{\tilde{I}_{k}}:=A_{J_{l}}^{I_{k}}$ cut-out from $A$ by choosing the selected tuples. Forgetting the partition gives the canonical bijection of sets $M_{\tilde{J}}^{\tilde{I}} \rightarrow M_{J}^{I}$. The multiplication of block matrices is defined by 
the usual matrix multiplication formula $(A B)_{J_{j}}^{I_{i}}=\sum_{l=1}^{r} A_{K_{l}}^{I_{i}} A_{J_{j}}^{K_{l}}$ if $A B$ and the sizes of subtuples for columns of $A$ and rows of $B$ match. One can further nest many levels of partitions (block-matrices of blockmatrices ...). Some considerations will not depend on whether we consider matrices in $R$ or block matrices, and then we'll just write $M_{J}^{I}$ etc. skipping the argument. More generally, the labels may be the objects in some abelian category $\mathcal{A}$, and entries $a_{j}^{i} \in \mathcal{A}(i, j) ; I$ will be the sum $\oplus_{i \in I} i$, hence $A: I \rightarrow J$. Ring multiplication is replaced by composition, defined when labels match.

Observation. Multiplication of block matrices commutes with forgetting (one level) of block-matrix structure. In other words we may multiply in stages (if working in $\mathcal{A}$ this is the associativity of $\oplus$ ). Corollaries:

(i) if $\tilde{I}=\tilde{J}$ then $M_{\tilde{I}}(R):=M_{\tilde{I}}^{\tilde{I}}(R)$ is a ring.

(ii) We can invert matrices in stages as well ('heredity').

(iii) The same for linear equations over noncommutative rings.

Any pair $(i, j) \in I \times J$ determines partitions $\tilde{I}=(i, \hat{i})$ and $\tilde{J}=$ $(j, \hat{j})$. For each $A$ in $M_{J}^{I}$ it induces a $2 \times 2$ block-matrix $A_{\tilde{J}}^{\tilde{I}}$. Reader should do the exercise of inverting that block matrix (with noncommutative entries), in terms of the inverses of blocks. As we will see, the $(i, j)$-quasideterminant of $A$ is the inverse of the $(j, i)$-entry of $A^{-1}$ if the latter is defined; though it may be defined when the latter is not.

16.1 The $(i, j)$-th quasideterminant $|A|_{i j}$ of $A$ is

$$
|A|_{i j}=a_{j}^{i}-\sum_{k \neq i, l \neq j} a_{l}^{i}\left(A_{\hat{j}}^{\hat{i}}\right)_{l k}^{-1} a_{j}^{k}
$$

provided the right-hand side is defined (at least in the sense of evaluating a rational expression, which will be discussed below). In alternative notation, the distinguished labels $i j$ may be replaced by a drawing of a box around the entry $a_{j}^{i}$ as in

$$
\left|\begin{array}{rrr}
a_{1}^{1} & a_{2}^{1} & a_{3}^{1} \\
a_{1}^{2} & a_{2}^{2} & a_{3}^{2} \\
a_{1}^{3} & a_{2}^{3} & a_{3}^{3}
\end{array}\right| \equiv\left|\begin{array}{lll}
a_{1}^{1} & a_{2}^{1} & a_{3}^{1} \\
a_{1}^{2} & a_{2}^{2} & a_{3}^{2} \\
a_{1}^{3} & a_{2}^{3} & a_{3}^{3}
\end{array}\right|_{32}
$$

At most $n^{2}$ quasideterminants of a given $A \in M_{n}(R)$ may be defined.

16.2 If all the $n^{2}$ quasideterminants $|A|_{i j}$ exist and are invertible then the inverse $A^{-1}$ of $A$ exist in $A \in M_{n}(R)$ and

$$
\left(|A|_{j i}\right)^{-1}=\left(A^{-1}\right)_{j}^{i}
$$


Thus we also have

$$
|A|_{i j}=a_{j}^{i}-\sum_{k \neq i, l \neq j} a_{l}^{i}\left|A_{\hat{j}}^{\hat{i}}\right|-1 a_{k l}^{k}
$$

16.3 On the other hand, the existence of inverse $A^{-1}$ does not imply existence of quasideterminants. For example, the unit $2 \times 2$ matrix over field $\mathbb{Q}$ has only 2 quasideterminants, not 4 . Or, worse, matrix $\left(\begin{array}{cc}3 & 2 \\ -2 & 3\end{array}\right)$ over ring $\mathbb{Z}\left[\frac{1}{7}\right]$ is invertible but no entry is, and hence no quasideterminants exist.

16.4 Quasideterminants are invariant under permutation of rows or columns of $A$ if we appropriately change the distinguished labels.

16.5 Suppose now we are given an equation of the form

$$
A x=\xi
$$

where $A \in M_{n}(R)$ and $x, \xi$ are $n$-tuples of indeterminates and free coefficients in $R$ respectively (they are column "vectors"). Then one can attempt to solve the system by finding the inverse of matrix $A$ and multiply the equation by $A^{-1}$ from the left, or one can generalize the Cramer's rule to the noncommutative setup.

Define thus $A(j, \xi)$ as the $n \times n$ matrix whose entries are the same as of $A$ except that the $j$-th column is replaced by $\xi$. Then the noncommutative left Cramer's rule says

$$
|A|_{i j} x^{j}=|A(j, \xi)|_{i j}
$$

and the right-hand side does not depend on $i$.

To see that consider first $n=2$ case:

$$
\begin{aligned}
& a_{1}^{1} x^{1}+a_{2}^{1} x^{2}=\xi^{1} \\
& a_{1}^{2} x^{1}+a_{2}^{2} x^{2}=\xi^{2}
\end{aligned}
$$

Then

$$
\begin{aligned}
|A|_{11} x^{1} & =a_{1}^{1} x^{1}-a_{2}^{1}\left(a_{2}^{2}\right)^{-1} a_{1}^{2} x^{1} \\
& =\left(\xi^{1}-a_{2}^{1} x^{2}\right)-a_{2}^{1}\left(a_{2}^{2}\right)^{-1} a_{1}^{2} x^{1} \\
& =\xi^{1}-a_{2}^{1}\left(a_{2}^{2}\right)^{-1} a_{2}^{2} x^{2}-a_{2}^{1}\left(a_{2}^{2}\right)^{-1} a_{1}^{2} x^{1} \\
& =\xi^{1}-a_{2}^{1}\left(a_{2}^{2}\right)^{-1} \xi^{2}=|A(1, \xi)|_{11} .
\end{aligned}
$$

The general proof is exactly the same, just one has to understand 
which indices are included or omitted in the sums involved:

$$
\begin{aligned}
|A|_{i j} x^{j} & =a_{j}^{i} x^{j}-\sum_{k \neq j, l \neq i} a_{k}^{i}\left(A_{\hat{j}}^{\hat{i}}\right)_{k l}^{-1} a_{j}^{l} x^{j} \\
& =\left(\xi^{i}-\sum_{h \neq j} a_{h}^{i} x^{h}\right)-\sum_{k \neq j, l \neq i} a_{k}^{i}\left(A_{\hat{j}}^{\hat{i}}\right)_{k l}^{-1} a_{j}^{l} x^{j} \\
& =\xi^{i}-\sum_{h \neq j, k \neq j, l \neq i} a_{k}^{i}\left(A_{\hat{j}}^{\hat{i}}\right)_{k l}^{-1} a_{h}^{l} x^{h}-\sum_{k \neq j, l \neq i} a_{k}^{i}\left(A_{\hat{j}}^{\hat{i}}\right)_{k l}^{-1} a_{j}^{l} x^{j} \\
& =\xi^{i}-\sum_{1 \leq h \leq n, k \neq j, l \neq i} a_{k}^{i}\left(A_{\hat{j}}^{\hat{i}}\right)_{k l}^{-1} a_{h}^{l} x^{h} \\
& =\xi^{i}-\sum_{1 \leq h \leq n, k \neq j, l \neq i} a_{k}^{i}\left(A_{\hat{j}}^{i}\right)_{k l}^{-1} a_{h}^{l} x^{h} \\
& =|A(j, \xi)|_{i j} .
\end{aligned}
$$

Similarly consider equation $\sum_{k} y^{k} B_{k}^{l}=\zeta^{l}$. Apparently the individual coefficients multiply $y^{k}$ from the right, but the combinatorics of matrix labels is organized as if we multiply By (alas, otherwise the rule of writing upper indices for rows would force us to write such equations upside-down!). The canonical antiisomorphism $R \rightarrow R^{\mathrm{op}}$ clearly sends any quasideterminant into the quasideterminant of the transposed matrix. Hence the left Cramer's rule implies the right Cramer's rule

$$
y^{j}\left|B^{T}\right|_{j i}=\left|(B(j, \zeta))^{T}\right|_{j i} .
$$

16.6 Row and column operations. Ordinary determinants do not change if we add a multiple of one row to another, and similarly for the columns.

We have to distinguish between left and right linear combinations.

If $|A|_{i j}$ is defined and $i \neq l$, then it is unchanged under left-row operation

$$
A^{l} \rightarrow A^{l}+\sum_{s \neq l} \lambda_{s} A^{s}
$$

Proof. We may assume $i=1$. Define the row matrix

$$
\vec{\lambda}=\left(\lambda_{2}, \ldots, \lambda_{n}\right) \text {. }
$$

Then $\vec{\lambda} T=\sum_{s \neq k} \lambda_{s} T^{s}$ for any matrix $T$ with row-labels $s=2, \ldots, n$. Then $\Lambda T=\sum_{s \neq k} \lambda_{s} T^{s}$. Assume the matrix $A$ is in the block-form written as

$$
\left(\begin{array}{cc}
a & \vec{b} \\
\vec{c}^{T} & D
\end{array}\right)
$$

with $a$ of size $1 \times 1$. Then

$$
\begin{aligned}
\left|\begin{array}{cc}
a+\vec{\lambda} \vec{c}^{T} & \vec{b}+\vec{\lambda} D \\
\vec{c}^{T} & D
\end{array}\right| & =a+\vec{\lambda} \vec{c}^{T}-(\vec{b}+\vec{\lambda} D) D^{-1} \vec{c}^{T} \\
& =a-\vec{b} D^{-1} \vec{c}^{T} .
\end{aligned}
$$


If we multiply the $l$-th row from the left by an invertible element $\mu$ then the quasideterminant $|A|_{i j}$ won't change for $i \neq l$ and will be multiplied from the left by $\mu$ if $i=l$. Actually, more generally, left multiply the $i$-th row by $\mu$ and the block matrix consisting of other rows by invertible square matrix $\Lambda$ (i.e. other rows can mix among themselves, and scale by different factors):

$$
A \rightarrow\left(\begin{array}{cc}
\mu & 0 \\
0 & \Lambda
\end{array}\right)
$$

Then $|A|_{i j}$ gets left multiplied by $\mu$ :

$$
\begin{aligned}
\left|\begin{array}{cc}
\mu a & \mu \vec{b} \\
\Lambda \vec{c}^{T} & \Lambda D
\end{array}\right|_{11} & =\mu a-\mu \vec{b}(\Lambda D)^{-1} \Lambda \vec{c}^{T} \\
& =\mu\left(a-\vec{b} D^{-1} \vec{c}^{T}\right)=\mu|A|_{i j} .
\end{aligned}
$$

16.7 Jacobi's ratio theorem. 59 Let $A$ be a matrix with possibly noncommutative entries such that the inverse $B=A^{-1}$ is defined. Choose some row index $i$ and some column index $j$. Make a partition of the set of row indices as $I \cup\{i\} \cup J$ and a partition of the set of column indices as $I^{\prime} \cup\{j\} \cup J^{\prime}$, with the requirements card $I=\operatorname{card} I^{\prime}$ and $\operatorname{card} J=\operatorname{card} J^{\prime}$. Then

$$
\left(\left|A_{I \cup\{i\}, I^{\prime} \cup\{j\}}\right|_{i j}\right)^{-1}=\left|B_{J^{\prime} \cup\{j\}, J \cup\{i\}}\right|_{j i} .
$$

Proof. The block decomposition of matrices does not change the multiplication, i.e. we can multiply the block matrices and then write out the block entries in detail, or we can write the block entries of the multiplicands in detail and then multiply and we get the same result. In particular, as $A=B^{-1}$, the block-entries of $A$ can be obtained by block-inversion of matrix $B$.

After possible permutation of labels, we may find the block-entry of the matrix $A=B^{-1}$ at the intersection of rows $I \cup\{i\}$ and columns $I^{\prime} \cup\{j\}$ by means of block-inverting the block matrix

$$
A=\left(\begin{array}{cc}
A_{I \cup\{i\}, I^{\prime} \cup\{j\}} & A_{I \cup\{i\}, J^{\prime}} \\
A_{J, I^{\prime} \cup\{j\}} & A_{J, J^{\prime}}
\end{array}\right)
$$

Then $A_{I \cup\{i\}, I^{\prime} \cup\{j\}}=\left(B_{I \cup\{i\}, I^{\prime} \cup\{j\}}-B_{I \cup\{i\}, J^{\prime}}\left(B_{J J^{\prime}}\right)^{-1} B_{J, I \cup\{i\}}\right)^{-1}$ or, equivalently,

$$
\left(A_{I \cup\{i\}, I^{\prime} \cup\{j\}}\right)^{-1}=B_{I \cup\{i\}, I^{\prime} \cup\{j\}}-B_{I \cup\{i\}, J^{\prime}}\left(B_{J J^{\prime}}\right)^{-1} B_{J, I^{\prime} \cup\{j\}}
$$


This is a matrix equality, and therefore it implies the equality of the $(i, j)$-th entry of both sides of the equation. We obtain

$$
\left(\left(A_{I \cup\{i\}, I^{\prime} \cup\{j\}}\right)^{-1}\right)_{i j}=b_{i j}-\sum_{k \in J^{\prime}, l \in J} b_{i, k}\left(B_{J J^{\prime}}\right)_{k l}^{-1} b_{l j} .
$$

Finish by applying the formula $|C|_{j i}=\left(C^{-1}\right)_{i j}$.

16.8 Muir's law of extensionality. [59] Let an identity $\mathcal{I}$ between quasiminors of a submatrix $A_{J}^{I}$ of a generic matrix $A$ be given. Let $K \cap I=\emptyset, L \cap J=\emptyset$ and $K=L$. If every quasiminor $\left|A_{V}^{U}\right|_{u v}$ of $A_{J}^{I}$ in the identity $\mathcal{I}$ is replaced by the quasiminor $\left|A_{V \cup L}^{U \cup K}\right|_{u v}$ of $A_{J \cup L}^{I \cup K}$ then we obtain a new identity $\mathcal{I}^{\prime}$ called the extensional to $\mathcal{I}$.

16.9 Quasitelescoping sum. Let $A=\left(a_{j}^{i}\right)$ be a generic $n \times n$ matrix. For any $k>2$, and $i, j \in\{1, k-1\}$ consider the quasiminor

$$
\left|A_{j, k, k+1, \ldots, n}^{i, k, k+1, \ldots, n}\right|_{i j}
$$

The quasitelescoping sum involves such minors:

$Q T\left(A_{1, \ldots, n}^{1, \ldots, n}\right)=\sum_{k=3}^{n}\left|A_{k-1, k, \ldots, n}^{1, k, \ldots, n}\right| 1, k-1\left|A_{k-1, k, \ldots, n}^{k-1, k, \ldots, n}\right|_{k-1, k-1}^{-1}\left|A_{1, k, \ldots, n}^{k-1, k, \ldots, n}\right|_{k-1,1}$

Then, by Muir's law and induction on $n$ we have

$$
Q T\left(A_{1, \ldots, n}^{1, \ldots, n}\right)=a_{1}^{1}-|A|_{11}
$$

For $n=3$ this is simply the identity obtained by extending by the third row and column the identity expressing the expansion of the $2 \times 2$ upper left quasiminor. Suppose now we have proved (22) for $n$. Take an $(n+1) \times(n+1)$-matrix $A$. Then, by induction, this is true for the submatrix

$$
A_{\hat{2}}^{\hat{2}}=A_{1,3, \ldots, n}^{1,3, \ldots, n}
$$

But

$$
\begin{aligned}
Q T & \left(A_{1, \ldots, n}^{1, \ldots, n}\right)=Q T\left(A_{1,3, \ldots, n}^{1,3, \ldots, n}\right)+\left|A_{2,3, \ldots, n}^{1,3, \ldots, n}\right|_{1,2}\left|A_{2,3, \ldots, n}^{2,3, \ldots, n}\right|_{2,2}^{-1}\left|A_{1,3, \ldots, n}^{2,3, \ldots, n}\right|_{2,1} \\
& =a_{1}^{1}-\left|A_{1,3, \ldots, n}^{1,3, \ldots, n}\right|_{11}+\left|A_{2,3, \ldots, n}^{1,3, \ldots, n}\right|_{1, k-1}\left|A_{2,3, \ldots, n}^{2,3, \ldots, n}\right|_{k-1, k-1}^{-1}\left|A_{1,3, \ldots, n}^{2,3, \ldots, n}\right|_{k-1,1} \\
& =a_{1}^{1}-\left|A_{1,2,3,3, \ldots, n}^{1,2, n}\right|_{11}
\end{aligned}
$$

where the last two summands were added, again by expansion of $2 \times 2$ upper left corner of $A$ and extending the identity by rows and columns $3, \ldots, n$. 
16.10 Homological relations. Start with the identity

$$
\left(a_{1}^{1}-a_{2}^{1}\left(a_{2}^{2}\right)^{-1} a_{1}^{2}\right)\left(a_{1}^{2}\right)^{-1}=-\left(a_{2}^{1}-a_{1}^{1}\left(a_{1}^{2}\right)^{-1} a_{2}^{2}\right)\left(a_{2}^{2}\right)^{-1}
$$

which in the quasideterminant language is

$$
\left|\begin{array}{ll}
a_{1}^{1} & a_{2}^{1} \\
a_{1}^{2} & a_{2}^{2}
\end{array}\right|\left(a_{1}^{2}\right)^{-1}=-\left|\begin{array}{ll}
a_{1}^{1} & a_{2}^{1} \\
a_{1}^{2} & a_{2}^{2}
\end{array}\right|\left(a_{1}^{2}\right)^{-1}
$$

and extend the latter by Muir's law, adding rows $3, \ldots, n$ to each minor of an $n \times n$ matrix $A$. Renaming the indices arbitrarily we obtain the row homological relations:

$$
|A|_{i j}\left|A_{\hat{j}^{\prime}}^{\hat{i}}\right|_{i^{\prime} j}^{-1}=-|A|_{i j^{\prime}}\left|A_{\hat{j}}^{\hat{i}}\right|_{i^{\prime} j^{\prime}}^{-1}
$$

for $j \neq j^{\prime}$. Similarly, starting with the identity

$$
\left(a_{2}^{1}\right)^{-1}\left(a_{1}^{1}-a_{2}^{1}\left(a_{2}^{2}\right)^{-1} a_{1}^{2}\right)-\left(a_{2}^{2}\right)^{-1}\left(a_{1}^{2}-a_{2}^{2}\left(a_{2}^{2}\right)^{-1} a_{1}^{2}\right)
$$

the same way we obtain the column homological relations.

$$
\left|A_{\hat{j}}^{\hat{i}^{\prime}}\right|_{i j^{\prime}}^{-1}|A|_{i j}=-\left|A_{\hat{j}}^{\hat{i}}\right|_{i^{\prime} j^{\prime}}^{-1}|A|_{i^{\prime} j}
$$

16.11 Laplace expansion for quasideterminants. Start with the identity

$$
\sum_{j} a_{j}^{i}\left(A^{-1}\right)_{k}^{j}=\delta_{k}^{i} .
$$

If $i \neq k$ and $A^{-1}$ exists then using $\left(A^{-1}\right)_{k}^{j}=|A|_{k j}^{-1}$ this specializes to

$$
\sum_{j} a_{j}^{i}|A|_{i j}^{-1}=1
$$

Multiply this equation from the right by $|A|_{i l}$ and split the sum into the part with $j \neq l$ and the remaining term:

$$
a_{l}^{i}+\sum_{j \neq l} a_{j}^{i}|A|_{i j}^{-1}|A|_{i l}=|A|_{i l}
$$

and apply the row homological relations (23) to obtain the following Laplace expansion for the $(i, j)$-th quasideterminant by the $k$-th row:

$$
a_{l}^{i}-\sum_{j \neq l} a_{j}^{i}\left|A_{\hat{l}}^{\hat{i}}\right|_{k j}^{-1}\left|A_{\hat{j}}^{\hat{i}}\right|_{k l}=|A|_{i l}
$$


Similarly, multiplying from the left the equation $\sum_{i}|A|_{i j}^{-1} a_{j}^{i}=1$ by $|A|_{l j}$ and splitting the sum into two terms we obtain

$$
a_{j}^{l}+\sum_{i \neq l}|A|_{l j}|A|_{i j}^{-1} a_{j}^{i}=|A|_{l j},
$$

which after the application of the column homological relations (24) gives the following Laplace expansion for the $(i, j)$-th quasideterminant by the $k$-th column:

$$
a_{j}^{l}-\sum_{j \neq l}\left|A_{\hat{j}}^{\hat{i}}\right| l k\left|A_{\hat{j}}^{\hat{l}}\right|_{i k}^{-1} a_{l}^{i}=|A|_{l j}
$$

Notice that the summation sign involves $(n-1)$ summands whereas the similar summation in the recursive formula (21) for quasideterminants involves $(n-1)^{2}$ summands.

16.12 23, 24] Let $R$ be a possibly noncommutative ring, and $\Sigma$ a given set of square matrices of possibly different (finite) sizes with entries in $R$. Map $f: R \rightarrow S$ of rings is $\Sigma$-inverting if each matrix in $\Sigma$ is mapped to an invertible matrix over $S$. A $\Sigma$-inverting ring map $i_{\Sigma}: R \rightarrow R_{\Sigma}$ is called Cohn localization (or universal $\Sigma$-inverting localization) if for every $\Sigma$-inverting ring map $f: R \rightarrow S$ there exist a unique ring map $\tilde{f}: R_{\Sigma} \rightarrow S$ such that $f=\tilde{f} \circ i_{\Sigma}$.

A set $\Sigma$ of matrices is called (upper) multiplicative if $1 \in \Sigma$ and, for any $A, B \in \Sigma$ and $C$ of right size over $R,\left(\begin{array}{cc}A & C \\ 0 & B\end{array}\right)$ is in $\Sigma$. If $\Sigma$ is the smallest multiplicative set of matrices containing $\Sigma_{0}$, then a map is $\Sigma_{0}$-inverting iff it is $\Sigma$-inverting. Inclusion $\Sigma_{0} \subset \Sigma$ makes every $\Sigma$-inverting map $f: R \rightarrow S$ also $\Sigma_{0}$-inverting. Conversely, if each of the diagonal blocks can be inverted, a block-triangular matrix can be inverted, hence $\Sigma_{0}$-inverting maps are $\Sigma$-inverting.

The universal $\Sigma$-inverting localization can be constructed by "invertive method", as follows. Represent $R$ as a free algebra $F$ on a generating set $\mathbf{f}$ modulo a set of relations $I$. For each quadratic matrix $A \in \Sigma$ of size $n \times n$, add $n^{2}$ generators $(A, i, j)$ to $\mathbf{f}$. This way we obtain a free algebra $F^{\prime}$ over some generating set $\mathbf{f}^{\prime}$. All $(A, i, j)$ for fixed $A$ clearly form a $n \times n$-matrix $A^{\prime}$ over $F^{\prime}$. Then $\Sigma^{-1} R=F^{\prime} / I^{\prime}$ where $I^{\prime}$ is the ideal generated by $I$ and by all elements of matrices $A A^{\prime}-I$ and $A^{\prime} A-I$ for all $A \in \Sigma$. Then $i_{\Sigma}: R \rightarrow \Sigma^{-1} R$ is the unique map which lifts to the embedding $F \hookrightarrow F^{\prime}$.

16.13 Warning. A naive approach to quotient rings, would be just adding new generators $a^{\prime}$ and relations $a a^{\prime}=a^{\prime} a=1$ for each 
$a \in R$ which needs to be inverted in first place. For any geometric purposes this would induce pretty unpredictable behaviour on modules etc. But suppose that one thinks as ring theorist and wants a quotient skewfield. After inverting all the nonzero elements one tries to invert all their sums which are nonzero and so on. The problem, even in principle, is that one may not know which elements from $m$-th step will be forced to zero by new relations a few steps later. So one should skip inverting some elements, as they will become zeros after a few steps when inverting other elements. There is no recipe which elements to leave out at each step. Moreover, for a given ring, there may be none (no quotient field) or multiple possibilities for such recipe. More precisely, given two embeddings $R \hookrightarrow K_{i}$ into skewfields $K_{1} \neq K_{2}$, there may be different smallest subskewfields $L_{i} \hookrightarrow K_{i}$ containing $R$.

16.14 Proposition. Let $\Sigma$ be multiplicative set of square matrices over $R$ and $f: R \rightarrow S$ a $\Sigma$-inverting map. Let $S(i, \Sigma) \subset R$ consists of all components of solutions over $S$ of all equations $f(A) x=f(b)$ where $A \in \Sigma, b$ is a column-vector over $R$ and $x$ a column of unknowns.

(i) $S(i, \Sigma)$ is a subring of $S$.

(ii) $S(i, \Sigma)$ coincides with the image of $R_{\Sigma}$ under the unique map $\tilde{f}: R_{\Sigma} \rightarrow S$ for which $f=\tilde{f} \circ i_{\Sigma}$.

In particular, if $f$ is 1-1 then $\tilde{f}$ is isomorphism and $i_{\Sigma}$ is 1-1.

(i) If components $x_{i}$ and $y_{j}$ of column vectors $x$ and $y$ over $S$ are in $S(i, \Sigma)$, with $f(A) x=b$ and $f(B) y=c$, then by possibly enlarging $x, y, b, c$ by zeroes and $A$ and $B$ by diagonal unit blocks, we may always make $i=j$ and $b$ and $c$ of the same length. Then $f\left(\begin{array}{cc}A & -A+B \\ 0 & B\end{array}\right)\left(\begin{array}{c}x+y \\ y\end{array}\right)=f\left(\begin{array}{c}b+c \\ c\end{array}\right)$ and as the left-hand side matrix is in $f(\Sigma)$ by multiplicativity, then $x_{i}+y_{i} \in S(i, \Sigma)$, as claimed. For $z$ a (row or column) vector consider the diagonal square matrix $\operatorname{diag}(z)$ with diagonal $z$. Then $\operatorname{diag}(z)(1,1, \ldots, 1)^{T}=$ $\left(z_{1}, \ldots, z_{n}\right)$. For a fixed $i$, there is a matrix $P_{i}$ such that $P_{i}\left(y_{1}, \ldots, y_{n}\right)^{T}=$ $\left(y_{i}, \ldots, y_{i}\right)^{T}$. Hence, $f\left(\begin{array}{cc}B & -\operatorname{diag}(c) P_{i} \\ 0 & A\end{array}\right)\left(\begin{array}{l}y \\ x\end{array}\right)=f\left(\begin{array}{l}0 \\ b\end{array}\right)$ has as the $j$-th component $\left(f(B)^{-1} f(c)\right)_{j}\left(f(A)^{-1} f(b)\right)_{i}$. But our blocktriangular matrix is in $\Sigma$, hence $x_{i} y_{j}$ is in $S(i, \Sigma)$. Similarly if we worked with algebras over $\mathbf{k}$ we could have taken some weights on diagonal instead of $\operatorname{diag}(c)$ to obtain any possible $\mathbf{k}$ linear combination of such.

(ii) The corestriction of $i$ onto $S(i, \Sigma)$ is also $\Sigma$-inverting. Hence there is a unique map form $R_{\Sigma}$. But there is by construction no smaller 
ring than $S(i, \Sigma)$ containing $f\left(R_{\Sigma}\right)$. As $i\left(R_{\Sigma}\right)$ is a ring they must coincide. If the map is 1-1 it has no kernel hence $\tilde{f}$ is an isomorphism.

16.15 Proposition. (left-module variant of P. M. CoHN 24, 2.1) If $\Sigma$ is multiplicative, then $\exists$ ! subfunctor $\sigma_{\Sigma}: R-\operatorname{Mod} \rightarrow R-\operatorname{Mod}$ of identity such that, as a subset, $\sigma_{\Sigma}(M)$ equals

$\left\{m \in M \mid \exists u=\left(u_{1}, \ldots, u_{n}\right)^{T} \in M^{\times n}, \exists i, m=u_{i}\right.$ and $\left.\exists A \in \Sigma, A u=0\right\}$

for every $M \in R-$ Mod. Moreover, $\sigma_{\Sigma}$ is an idempotent preradical.

Proof. 1. $\sigma_{\Sigma}(M)$ is an $R$-submodule of $M$. It is sufficient to show that for any $r \in R, m, m^{\prime} \in \sigma_{\Sigma}(M)$ the left linear combination $m+$ $r m^{\prime} \in \sigma_{\Sigma}(M)$. Choose $A, B \in \Sigma, A u=0, B v=0, u \in M^{\times k}, v \in M^{\times l}$, $m=u_{i}, m^{\prime}=v_{j}$. We may assume $k=l, i=j$, hence $m+r m^{\prime}=(u+$ $r v)_{i}$, by adjusting $A, B, u, v$. For example, $\tilde{A}:=\operatorname{diag}\left(I_{s}, A, I_{t}\right) \in \Sigma$ and $\tilde{u}:=\left(0_{s}, u_{1}, \ldots, u_{k}, 0_{t}\right)^{T} \in M^{s+k+t}$ satisfy $\tilde{A} \tilde{u}=0$ with $m=\tilde{u}_{i+s}$. Then $\left(\begin{array}{cc}A & -A r \\ 0 & B\end{array}\right) \in \Sigma$ and $\left(\begin{array}{cc}A & -A r \\ 0 & B\end{array}\right)\left(\begin{array}{c}u+r v \\ v\end{array}\right)=\left(\begin{array}{l}0 \\ 0\end{array}\right)$.

2. $M \mapsto \sigma_{\Sigma}(M)$ extends to a unique subfunctor of identity. If $m=$ $u_{i} \in \Sigma$ for some $i$ and $A\left(u_{1}, \ldots, u_{k}\right)^{T}=0$ then $A\left(f\left(u_{1}\right), \ldots, f\left(u_{k}\right)\right)^{T}=$ 0 whenever $f: M \rightarrow N$ is $R$-module map. As $f(m)=f\left(u_{i}\right)$ this proves that $f\left(\sigma_{\Sigma}(M)\right) \subset \sigma_{\Sigma}(f(M))$ as required.

3. $\sigma_{\Sigma}(M)$ is a preradical: $\sigma_{\Sigma}\left(M / \sigma_{\Sigma}(M)\right)=0$. If $m \in \sigma_{\Sigma}(M)$, then $\exists u_{1}, \ldots, u_{k} \in M, \exists A \in \Sigma, \exists p \leq k$, such that $A \vec{u}^{T}=0 \bmod \sigma_{\Sigma}(M)$ and $m=u_{p}$ where $\vec{u}:=\left(u_{1}, \ldots, u_{k}\right)$. Hence $\exists v_{1}, \ldots, v_{k} \in \sigma_{\Sigma}(M)$ such that $A \vec{u}^{T}=\left(v_{1}, \ldots, v_{k}\right)^{T}$ and there are matrices $B_{1}, \ldots, B_{k}$, where $B_{s}$ is of size $h_{s} \times h_{s}$, and vectors $\left(w_{1 s}, \ldots, w_{h_{s} s}\right)$ of size $h_{s}$, such that $B_{s}\left(w_{1 s}, \ldots, w_{h s}\right)^{T}=0$ for all $s$; and we have that $v_{i}=w_{s_{i} i}$ for some correspondence $i \mapsto s_{i}$. Let $\vec{w}=\left(w_{11}, \ldots, w_{h_{1} 1}, w_{12}, \ldots, w_{k s_{k}}\right)$. Let matrix $J=\left(J_{j}^{i}\right)$ be defined by $J_{s_{i}}^{i}=1$ for each $i$ and all other entries are 0 . This matrix by construction satisfies $J \vec{w}^{T}=\vec{v}$. Define also the block matrix $B:=\operatorname{diag}\left\{B_{1}, \ldots, B_{k}\right\}$. Clearly $B \vec{w}^{T}=0$ by construction and $B \in \Sigma$ by multiplicativity of $\Sigma$. In this notation the summary of just said is encoded in this block identity

$$
\left(\begin{array}{cc}
A & -J \\
0 & B
\end{array}\right)(\vec{u}, \vec{v})^{T}=0, \quad\left(\begin{array}{cc}
A & -J \\
0 & B
\end{array}\right) \in \Sigma, m=u_{p}
$$

4. $\sigma_{\Sigma}\left(\sigma_{\Sigma}(M)\right)=\sigma_{\Sigma}(M)$. If $m=u_{i}$ for some $i$ and $A\left(u_{1}, \ldots, u_{k}\right)^{T}=$ 0 for some $A \in \Sigma$ with all $u_{j} \in \sigma_{\Sigma}(M)$, then in particular, all $u_{j} \in M$. Q.E.D.

Exercise. Let $\Sigma, \Sigma^{\prime}$ be multiplicative sets of matrices over $R$. 
If for every $A \in \Sigma$ there are permutation matrices $w, w^{\prime} \in G L(k, \mathbb{Z})$ such that $w A w^{\prime} \in \Sigma^{\prime}$ then $\sigma_{\Sigma^{\prime}}(M) \subset \sigma_{\Sigma}(M)$ for all $M$.

16.16 Warning. $\sigma_{\Sigma}$ is not necessarily left exact. Equivalently, the associated torsion theory is not always hereditary (i.e. a submodule of a $\sigma_{\Sigma^{-}}$-torsion module is not necessarily $\sigma_{\Sigma^{-}}$-torsion). Hereditary torsion theories correspond to Gabriel localizations.

16.17 The main problem with Cohn localization in ring-theoretic practice is that it is usually hard to determine the kernel of the localization map $i_{\Sigma}$. In geometric circumstances, the lack of flatness of the inverse image functor $Q^{*}$ is the main problem. It is also not clear when the flatness is lost. There are several characterizations under additional assumptions, usually assuming that we invert all matrices related to a certain ideal, rather than an explicitly given set of matrices which is more often the case in geometry.

16.17a The advantage is that this localization may be fully described in terms of the ground ring. Gabriel localization has flatness but elements of $S$ are not really inverted. In the definition of quasicoherent sheaf one may replace localizations of modules by tensor products with localized ring, as if it were a perfect localization. Such a setup has some good general properties, as noticed by Orlov [83]).

16.18 Quasideterminants vs. Cohn localization. Quasideterminants are given by explicit formulas. It is more manageable to invert them than matrices when the inverse can not be expressed in terms of them. But this is generic, as even the unit matrix does not have all quasideterminants. Hence one combines a process, to have good ring and still to have more explicit handle. The combination is however not necessarily a Cohn localization.

So, let $\Sigma$ be as before. For each $A \in \Sigma$ and pair $(i j)$ such that $\mid A_{\mid} i j$ exists and is nonzero add a variable $B_{i j}$ and require $B_{i j} \mid A_{\mid} i j=$ $A_{\mid} i j \mid B_{i j}=1$. One obtains a localization $j: R \rightarrow R_{\Sigma}^{q 0}$. Then one inverts $j(\Sigma)$ by the Cohn method, which amounts to adding formal variables just for those entries which are not added before as quasideterminants, and adding relations for them. The result is some localization $i_{\Sigma}^{q}: R \rightarrow R_{\Sigma}^{q}$ which is $\Sigma$-inverting and clearly a quotient ring of the Cohn localization. If $i_{\Sigma}^{q}$ is injective, it is just the Cohn localization.

There are obvious variants of this method, cf. warning $\mathbf{1 6 . 1 3}$ for a hint, and recall that quasideterminants may be defined inductively by size. Some rings may be quotiented by ideals to get e.g. commutative or Ore domains. A quasideterminant may be proven to be nonzero, 
as its image in the quotient is nonzero, which is a good procedure for some concrete $\Sigma$ (cf. 106, Th.7).

We have seen that for the usual descent of quasicoherent sheaves one needs flatness, which is lacking for Cohn localization. We would still like, in the spirit of an example [106], Th.7, to be able to consider some global noncommutative spaces where the local coordinates are compared using Cohn localizations. ORLOv ([83]) defines quasicoherent sheaves on ringed sites; and considers an interesting Grothendieck topology on (noncommutative) Aff. As Cohn localizations always define a ring, and non-perfect exact localizations define only a category, one should emphasize the structure sheaf rather than only categories when trying to construct global spaces related locally to the Cohn localizations of other spaces.

Acknowledgements. My biggest thanks go to Prof. A. RANICKI, who patiently encouraged the completion of this article.

Many thanks to Prof. J. RoBBin for trying to improve my writing style, to be straight, explicit and to avoid a too frequent use of 'it is wellknown that'. Thanks to Indiana University, Bloomington where a piece of my Wisconsin student 'preprint' started growing into this paper, and Prof. V. LunTs of Indiana for insights into mathematics and his manuscripts. I thank the Rudjer Bošković Institute, Zagreb, and the Max Planck Institute, Bonn, for allowing me to use a big chunk of my research time there to complete this survey. Finally, my apology to the reader that I needed much more space and time for this article to be polished and balanced than I could deliver.

Bibliography. We try to give a few of the most useful references for a reader who has similar geometric needs to the author. The bibliography is intentionally incomplete. For the benefit of the geometrically oriented reader, we used the following preference criteria: geometrically motivated, historically important, readable (for the author at least), irreplacable. The literature which is obscure to me in a major way is naturally not in the list. However I mention here some undoubtedly important alternative works by listing only Math. Reviews code. For ring theorists there is a monograph on torsion theories by Golan MR88c:16934 and on localization by Jatageonkar MR88c:16005 and by Golan MR51:3207.

Many equivalent approaches to Gabriel localization have been multiply discovered (Goldman (1969) [44, Silver (1967) MR36:205, Maranda (1964) MR29:1236 etc.) in various formalisms, e.g. torsion theories (the term is basically from Dickson (1965) MR32:2472).

Despite their historical importance, we ignore these, and recommend the systematic treatment in Gabriel's thesis [34] as well as the books [52, 87, 114 and Ch. 6 of [20]. For abelian categories see [17, 32, 34, 36, 37, 52, 87, 109, 48, 127; for localization in abelian categories see books [17, 32, 52, 87, 109. Other longer bibliographies are in [32, 52, 60, 87]. Abbrev.: LMS = London 
Mathematical Society, MPI = Max Planck Inst. preprint (Bonn). \& for Springer series: GTM (Graduate Texts in Math.), LNM (Lecture Notes in Math.), Grundl.MW (Grundlehren Math.Wiss.).

\section{References}

[1] P. Ara, M. Martin, Local multipliers of $C^{*}$-algebras, Springer, London 2003.

[2] M. ARTin, Noncommutative deformations of commutative rings, preprint 1994.

[3] M. Artin, J. J. Zhang, Noncommutative projective schemes, Adv. Math. 109 (1994), no. 2, pp. 228-287.

[4] K. Asano, Über die Quotientenbildung von Schiefringen, (German) J. Math. Soc. Japan 1, (1949), pp. 73-78.

[5] M. BARR, Composite triples and derived functors, in Seminar on triples and categorical homology theory, H. Appelgate et al. eds. LNM 80, Springer 1969.

[6] M. Barr, C. Wells, Toposes, triples and theories, Grundl.MW 178, Springer 1985.

[7] J. Beck, Distributive laws, in Seminar on triples and categorical homology theory, H. Appelgate et al. eds. LNM 80, Springer 1969.

[8] J. M. BECK, Triples, algebras and cohomology, Ph.D. Thesis, Columbia Univ. 1963.

[9] KaI Behrend, Differential Graded Schemes I: Perfect resolving algebras, math.AG/0212225 II: The 2-category od dg schemes, math.AG/0212226.

[10] S. Beilinson, V. Drinfeld, Chiral algebras, (large, $>300$ pp.) preprint (1995-).

[11] A. I. Bondal, M. M. Kapranov, Representable functors, Serre functors, and reconstructions, Izv. Akad. Nauk SSSR Ser. Mat. 53 (1989), no. 6, 1183-1205, 1337; translation in Math. USSR-Izv. 35 (1990), no. $3,519-541$

[12] A. Bondal, D. Orlov, Derived categories of coherent sheaves, math. AG/0206295.

[13] A. Bondal, M. VAn Den Bergh, Generators and representability of functors in commutative and noncommutative geometry, Mosc. Math. J. 3 (2003), no. 1, 1-36, 258; math.AG/0204218.

[14] D. Ben-Zvi, T. Nevins, From solitons to many-body systems, math.AG/0310490 
[15] G. M. Bergman, The diamond lemma for ring theory, Adv.M. 29 (1978), 178-219.

[16] J. Bernstein, V. LunTs, Equivariant sheaves and functors, LNM 1578 (1994).

[17] F. Borceux, Handbook of categorical algebra, 3 vols. Enc. of Math. and its Appl. 50, Cambridge Univ. Press, 1994.

[18] A. Borel, Linear Algebraic Groups, 2nd edition, GTM 126, Springer 1991.

[19] N. Bourbaki, Elements de mathematique: Algebre commutative, II 1964-9 Hermann, Paris, (engl. transl. Commutative Algebra II, Addison Wesley 1972).

[20] I. Bucur, A. Deleanu, Introduction to the theory of categories and functors, Pure Appl. Math. XIX, Wiley 1968.

[21] T. Brzeziński, S. Majid, Coalgebra bundles, Comm. Math. Phys. 191 (1998), no. 2, pp. 467-492.

[22] P. CARTiER, A mad day's work: from Grothendieck to Connes and Kontsevich - the evolution of concepts of space and symmetry, Bull. AMS 38, No.4, pp. 389-408, 2001.

[23] P. M. Cohn, Free rings and their relations, Academic Press 1971.

[24] P. M. CoHN, Inversive localization in Noetherian rings, Comm. Pure. Appl. Math. 26 (1973), pp. 679-691.

[25] P. M. Conn, Algebra, Vol. 3, 2nd ed. John Wiley and Sons, Chichester, 1991.

[26] A. Connes, Noncommutative geometry, Academic Press, New York 1994.

[27] M. Demazure, P. Gabriel, Groupes algébriques I, 1970 (French), Introduction to algebraic geometry and algebraic groups (Engl.transl. Ch.13), North Holland Publ.Co. 1980.

[28] J. Dixmier, Enveloping algebras, North Holland (1977) (enhanced version AMS 1997).

[29] V. DRINFELD, DG quotients of DG categories, math.KT/0210114.

[30] C. Ehresmann, Catégories et structure, Dunod 1965.

[31] V. P. Elizarov, Kol'ca častnyh (russian), Alg. i logika 8 (4) 1969, pp. 381-424; K obščej teorii kolec častnyh, Sib. Mat. Ž 11 (3), 1970 pp. 526-546; Sil'nye prekručenija i sil'nye fil'try, moduli i kol'ca častnyh, Sib. Mat. Ž 14 (3), 1973, pp. 549-559.

[32] C. Faith, Algebra I,II, Grundleheren Math. Wiss. 190, 191, Springer $1973,1976,1981$. 
[33] C. FAITH, Rings and things and a fine array of twentieth century associative algebra, Math. Surv. Monographs 65, AMS 1999.

[34] P. Gabriel, Des catégories abéliennes, Bull. Soc. Math. France 90 (1962), 323-448;

[35] P. Gabriel, M. Zisman, Calculus of fractions and homotopy theory, Springer 1967.

[36] S. I. Gelfand, Yu. I. Manin, Homological algebra, Springer.

[37] S. I. Gelfand, Yu. I. Manin, Methods of homological algebra, Springer 1996; translated from the Russian original, Nauka 1988.

[38] I. M. Gel'fand, V. S. Retakh, Determinants of matrices over noncommutative rings, Fun. Anal. Appl. vol 25 (1991), no. 2, pp. 91-102. transl. 21 (1991), pp. 51-58.

[39] I. M. Gel'fand, V. S. Retakh, A theory of noncommutative determinants and characteristic functions of graphs, Fun. Anal. Appl. vol. 26 (1992), no.4, pp. 231-246.

[40] I. M. Gel'fand, V. S. Retakh, Quasideterminants I, Selecta Mathematica, New Series 3 (1997) no.4, pp. 517-546; q-alg/9705026

[41] I. Gelfand, S. Gelfand, V. Retakh, R. Wilson, Quasideterminants, arXiv:math.QA/0208146

[42] J. Giraud, Cohomologie non abélienne, Grundl. Math. Wiss. 179, Springer 1971.

[43] J. S. Golan, J. Raynaud, F. van Oystaeyen, Sheaves over the spectra of certain noncommutative rings, Comm. Alg. 4(5), (1976), pp. 491-502.

[44] O. Goldman, Rings and modules of quotients, J. Algebra 13 (1969), pp. 10-47.

[45] K. R. Goodearl, R. B. Warfield JR., An introduction to noncommutative Noetherian rings, LMS Student Texts, Vol. 16, Cambridge Univ. Press 1989.

[46] A. GRothendieck, Éléments de géométrie algébrique. IV. Étude locale de schémas et de morphismes de schémas, Publ. Math. IHES 32 (1967)

[47] A. Grothendieck et AL., Revêtements étales et groupe fondamental, Séminaire de Géometrie Algébrique du Bois Marie 1960-1961 (SGA 1), LNM 224, Springer (1971), retyped as: arXiv:math.AG/0206203

[48] A. Grothendieck, Sur quelques points d'algèbre homologique, Tôhoku Math. J. (2) 91957 119-221.

[49] R. Hartshorne, Algebraic geometry, Springer, GTM 52, 1977.

[50] R. HARTSHORNe, Residues and duality (with an appendix by P. Deligne), Springer 1966. 
[51] T. J. Hodges, S. P. Smith, Sheaves of noncommutative algebras and the Beilinson-Bernstein equivalence of categories, Proc. AMS, 92, N. 3, 1985 .

[52] P. Jara, A. Verschoren, C. Vidal, Localization and sheaves: a relative point of view, Pitman Res. Notes in Math. 339, Longman 1995.

[53] P. T. Johnstone, Topos theory, LMS Monographs 10, Acad. Press 1977;

Sketches of an elephant: a topos theory compendium, 3 vols, Oxford Univ. Press 2002.

[54] M. Kapranov, Noncommutative geometry based on commutator expansions, J. Reine Angew. Math. 505 (1998), pp. 73-118; arXiv:math.AG/9802041

[55] A. Kapustin, A. Kuznetsov, D. Orlov, Noncommutative instantons and twistor transform, Comm. Math. Phys. 221 (2001), pp. 385-432; arXiv:hep-th/0002193

[56] M. Kontsevich, Deformation quantization for algebraic varieties, math.QA/0106006

[57] M. Kontsevich, A. L. Rosenberg, Noncommutative smooth spaces, The Gelfand Math. Seminars, 1996-1999, Birkhäuser 2000, pp. 85-108; arXiv:math.AG/9812158

[58] M. Kontsevich, Y. Soibelman, Homological mirror symmetry and torus fibrations, Symplectic geometry and mirror symmetry (Seoul, 2000), pp. 203-263, math.SG/0011041.

[59] D. Krob, B. LeClerc, Minor identities for quasi-determinants and quantum determinants, Comm.Math.Phys. 169 (1995), pp. 1-23.

[60] J. LAMBEK, Torsion theories, additive semantics and rings of quotients, LNM 177.

[61] N.P. Landsman, Bicategories of operator algebras and Poisson manifolds, Math. physics in math. \& physics (Siena, 2000), Fields Inst. Comm. 30, 271-286, AMS 2001.

[62] N.P. LANDSMAN, Math. topics between classical \& quantum mechanics, Springer 1998.

[63] O.A. LAUdAL, Noncommutative algebraic geometry, preprint MPI 2000115.

[64] D.A. LEITES, Introduction to the theory of supermanifolds (Russian), Usp. Mat. Nauk. 35 (1980), pp. 3-57.

[65] V. A. LunTs, Deformations of quasicoherent sheaves of algebras, J. Alg. 259 (2003), pp. 59-86; arXiv:math.AG/0105006 
[66] V. A. Lunts, A. L. Rosenberg, Differential calculus in noncommutative algebraic geometry, Max Planck Institute Bonn preprints:

I. D-calculus on noncommutative rings, MPI 96-53;

II. D-calculus in the braided case. The localization of quantized enveloping algebras, MPI 96-76, Bonn 1996.

[67] V. A. Lunts, A. L. Rosenberg, Differential operators on noncommutative rings, Sel. Math. N. S. 3 (1997), pp. 335-359.

[68] V. A. Lunts, A. L. Rosenberg, Localization for quantum groups, Selecta Math. (N.S.) 5 (1999), no. 1, pp. 123-159.

[69] V. A. Lunts, A. L. Rosenberg, Localization for quantum groups II, manuscript.

[70] G. LuszTig, Introduction to quantum groups, Progress in Math. 110, Birkhäuser, Boston 1993.

[71] S. MAC LANE, Categories for the working mathematician, GTM 5, Springer 1971.

[72] S. Mac Lane, I. MoerdiJk, Sheaves in geometry and logic, Springer 1992.

[73] YU. I. MANIN, Quantum groups \& non-commutative geometry, CRM, Montreal 1988.

[74] Yu. I. Manin, Moduli, motives, mirrors, pp. 53-73, Proc. 3rd Eur. Cong. Math. Barcelona July 10-14, 2000, Prog. Math. 201; math. AG/0005144.

[75] Yu. I. Manin, Topics in noncommutative geometry, Princeton Univ. Press 1991.

[76] H. Fausk, Po Hu, J. P. May, Isomorphisms between left and right adjoints, Theory Appl. Categ. 11 (2003), pp. 107-131 (electronic).

[77] J. C. MCConnell, J. C. RoBson, Noncommutative noetherian rings, 1987.

[78] D. C. Murdoch, F. van Oystaeyen, Noncommutative localization and sheaves, J. Alg. 38 (1975), pp. 500-515.

[79] S. Montgomery, H.-J. Schneider, Hopf crossed products, rings of quotients and prime ideals, Adv. Math. 112 (1995), pp. 1-55.

[80] J. Ndirahisha, F. VAn Oystaeyen, Grothendieck representations of categories and canonical noncommutative topologies, K-theory 25 (2002), pp. 355-371.

[81] A. Neeman, The Grothendieck duality theorem via Bousfield's techniques and Brown representability, J. AMS 9 (1996), pp. 205-236.

[82] O. ORE, Linear equations in noncomm. fields, Ann. Math. 32 (1931), pp. $463-477$. 
[83] D. O. OrLov, Quasi-coherent sheaves in commutative and noncommutative geometry, Izvestiya: Math. 67:3 (2003), pp. 119-138.

[84] D. O. Orlov, Derived categories of coherent sheaves and equivalences between them, (Russian) Uspehi Mat. Nauk 58 (2003), no. 3, pp. 89-172.

[85] C. J. PAPPaCena, Frobenius bimodules between noncommutative spaces, math.QA/0304386

[86] A. Polishchuk, M. Rothstein, Fourier transform for D-algebras, math.AG/9901009.

[87] N. Popescu, Abelian categories with applications to rings and modules, London Math. Soc. Monographs 3, Academic Press (1973).

[88] L. Popescu, N. Popescu, Theory of categories, Bucuresti \& Alphen: Editura Academiei \& Sijthoff \& Noordhoff International Publishers, 1979.

[89] T. Porter, $\mathcal{S}$-categories, $\mathcal{S}$-groupoids, Segal categories and quasicategories, math.AT/0401274

[90] N. Reshetikhin, A. Voronov, A. Weinstein, Semiquantum geometry, Algebraic geometry 5, J. Math. Sci. 82 (1996), no. 1, pp. 3255-3267; q-alg/9606007

[91] A. L. Rosenberg, Noncomm. local algebra, Geom. Fun. Anal. 4 (5), $1994,545-585$.

[92] A. L. Rosenberg, The spectrum of abelian categories and reconstruction of schemes, in Rings, Hopf algebras, and Brauer groups (Antwerp/Brussels, 1996), pp. 257-274, Lec. Notes Pure Appl. Math. 197, Dekker, NY 1998. ('96 version: MPI-1996-108).

[93] A. L. Rosenberg, Noncommutative schemes, Comp. Math. 112 (1998), pp. $93-125$.

[94] A. L. Rosenberg, Noncommutative spaces and schemes, MPI-1999-84, Bonn 1999.

[95] A. L. Rosenberg, Spectra related with localizations, MPI-2003-112, Bonn 2003.

[96] A. L. Rosenberg, Non-commutative affine semischemes and schemes, Seminar on supermanifolds No. 26, edited by D. Leites, Dept. of Math., Univ. of Stockholm 1988.

[97] A. L. Rosenberg, Noncommutative algebraic geometry and representations of quantized algebras, MAIA 330, Kluwer 1995.

[98] A. L. Rosenberg, Spectra of noncommutative spaces, MPI-2003-110, Bonn 2003.

[99] A. L. RosenBerg, Underlying spectra of noncommutative schemes, MPI-2003-111, Bonn 2003. 
[100] L. Rowen, Ring theory, 2 vols, Acad. Press (Pure \& Applied Math. $127 \& 128), 1988$.

[101] A. C.Da Silva, A. Weinstein, Geometric models for noncommutative algebra, UC Berkeley, AMS, 1999, 184 pp.

[102] Z. ŠKodA, Cyclic structures on simplicial objects from comonads, preprint.

[103] Z. ŠKodA, Coherent states for Hopf algebras, math.QA/0303357

[104] Z. ŠKODA, Globalizing Hopf-Galois extensions, preliminary version.

[105] Z. ŠKoDA, Localized coinvariants I,II, preprints.

[106] Z. ŠKODA, Localizations for construction of quantum coset spaces, in "Noncommutative geometry and quantum groups", P. M. Hajac, W. Pusz eds. Banach Centre Publications vol.61, pp. 265-298, Warszawa 2003; arXiv:math.QA/0301090.

[107] Z. ŠKODA, Quantum bundles using coactions and localization, in preparation.

[108] P. S. Smith, Subspaces of non-commutative spaces, preprint math.QA/0104033

Integral non-commutative spaces, preprint math.QA/0104046

Maps between non-commutative spaces, preprint math.QA/0208134

[109] P. S. SмIтH, Non-commutative algebraic geometry, preprint notes, U. Wash. 2000.

[110] Y. SoIBelman, Quantum tori, mirror symmetry and deformation theory, Lett. Math. Phys. 56 (2001), no. 2, 99-125 math.QA/0011162.

with P. BREssler, Mirror symmetry and deformation quantization, hep-th/0202128.

with V. VOLOGODSKY, Non-commutative compactifications and elliptic curves, math.AG/0205117.

[111] J. T. Stafford, Noncommutative projective geometry, Proceedings of the ICM, Beijing 2002, vol. 2, pp. 93-104; math.RA/0304210

[112] J. T. Stafford, T. A. Nevins, Sklyanin algebras and Hilbert schemes of points, math.AG/0310045

[113] J. T. Stafford, M. VAn Den Bergh, Noncommutative curves and noncommutative surfaces, Bull. AMS 38 (2001), pp.171-216.

[114] Bo Stenström, Rings of quotients, Grundlehren Math. Wiss. 217, Springer 1975.

[115] R. Street, The formal theory of monads, Journal of Pure and Applied Algebra 2 (1972), 149-168;

part II (with S. LACK) J. Pure Appl. Algebra 175 (2002), no. 1-3, 243265. 
[116] B. Toen, Homotopical and higher categorical structures in algebraic geometry, math.AG/0312262

Affine stacks (Champs affines) math.AG/0012219

[117] B. Toen, G. Vezzosi, Algebraic geometry over model categories etc. math.AG/0110109, math.AG/0207028, math.AG/0210407, math.AG/0212330, math.AT/0309145.

[118] M. VAN DEN BERGH, Blowing up of non-commutative smooth surfaces, Mem. Amer. Math. Soc. 154, 2001.

[119] A. VAN DEN Essen, Algebraic microlocalization and modules with regular singularities over filtered rings, Handbook of algebra, Vol. 1, 813-840, North-Holland, 1996.

[120] A. Verschoren, Sheaves and localization, J. Algebra 182 (1996), no. 2, pp. 341-346.

[121] F. van Oystaeyen, Algebraic geometry for associative algebras, Marcel Dekker 2000.

[122] F. van Oystaeyen, A. Verschoren, Reflectors and localization, application to sheaf theory, Lec. Notes in Pure. Appl. Math. 41, M. Dekker 1979.

[123] F. Van Oystaeyen, L. Willaert, Grothendieck topology, coherent sheaves and Serre's theorem for schematic algebras, J. Pure Appl. Alg. 104 (1995), pp. 109-122.

[124] F. van Oystaeyen, L. Willaert, Cohomology of schematic algebras, J. Alg. 185 (1996), pp. 74-84.

[125] A. Vistoli, Notes on Grothendieck topologies, fibered categories and descent theory.

[126] C. E. WaTts, Intrinsic characterization of some additive functors, Proc. AMS 11 (1960), pp. 1-8.

[127] C. Weibel, Homological algebra, Cambridge Univ. Press 1994.

[128] R. Wisbauer, Module and comodule categories - a survey, Proc. of the Math. Conf. Birzeit/Nablus, 1998, World Scientific (2000), pp. 277-304.

[129] H. WolfF, $\mathcal{V}$-localizations and $\mathcal{V}$-monads, J. Alg. 24 (1973), pp. 405438.

[130] A. YeKUTIELI, On deformation quantization in algebraic geometry, math.AG/0310399

[131] A. YeKutieli, J. L. Zhang, Dualizing complexes and perverse sheaves on noncommutative ringed schemes, math.QA/0211309 


\section{Index}

$\mathfrak{I S p}, 50$

$\dashv, 34$

$\mathbb{Z}$-filtered object, 55

$\mathcal{C}_{\mathcal{D}}(R, S), 15$

ISp, 45

$\mathfrak{r} \mathfrak{s p}, 45$

$\operatorname{Aff}(\tilde{A}), 45$

Fas $\mathcal{C}, 8$

$\mathrm{Pa} \mathcal{E}, 11$

cAff, 45

1-cell, 53

2-category, 53

strict, 53

2-cell, 53

abstract affine scheme, 45

action of monad, 33

adjoint functors, 34

adjunction, 34

admissible subset, 60

affine functor, 52

algebra over monad, 33

algebraic microlocalization, 55

almost flat, 52

associated graded algebra, 56

Barr-Beck lemma, 36

bicategory, 53

block matrix, 72

bounded poset, 66

category of paths, 11

coequalizer, 4

cofree G-comodule, 34

coherence, 32

column homological relations, 78

commutative localization, 44

comonad, 33

comparison functor, 34

compatible calculi, 65

completely prime ideal, 47

configuration category, 38

conservative family, 37 continuous functor, 52

coproduct

of comonad, 33

counit of adjunction, 34

counit of comonad, 33

deformation, 55

Deligne's theorem, 50

derived algebraic geometry, 45

differential Ore condition, 65

distributive law, 39

Eilenberg-Moore, 34

equalizer, 4

exhaustive filtration, 55

extending calculi, 65

extending Ore property, 24

extension of scalars, 50

filter, 66

uniform, 66

filtered-relative Ore condition, 58

first order differential calculus, 64

flat functor, 52

flat morphism, 37

FODC, 64

fork diagram, 4

Frobenius module, 54

Gabriel filter, 67

geometric affine schemes, 44

gluing procedure, 44

Godement product, 32

Grothendieck (pre)topology, 46

Grothendieck category, 8

homological relations, 78

horizontal composition, 32

idempotent radical, 69

identity arrow, 53

inclusion preorder, 66

initial object, 4

injective hulls, 3 
interchange law, 33

Jacobi's ratio theorem, 76

join, 66

kernel, 4

Laplace expansion for quasideterminants, 78 lattice, 66

left calculus of fractions, 14

left Cramer's rule, 74

left denominator set, 13

left Ore set, 13

left orthogonal subcategory, 70

Leibnitz identity, 64

localizing subcategory, 71

localizing to open subscheme, 50

meet, 66

module over monad, 33

monad, 33

associated to adjunction, 34

monoid, 13

monoidal category

symmetric, 45

morphism of monads, 35

Muir's law of extensionality, 77

multiplicative set

of matrices, 79

multiplicative subset, 13

multiplicative system, 14

multiplicatively generated, 13

Ore localization, 16

partitioned matrix, 72

pentagon axiom, 32

Poisson algebra, 62

Poisson bracket, 62

preradical, 69

preserving limits, 5

prime ideal, 47

principal open set, 47

pseudofunctor, 38

quasicompact relative noncommutative scheme, 52

quasitelescoping sum, 77 radical, 69

radical filter, 67

reflecting limits, 5

regular element, 59

replete subcategory, 70

restriction of scalars, 50

right Cramer's rule, 75

right orthogonal subcategory, 70

ring of fractions, 16

row and column operations, 75

row homological relations, 78

saturated multiplicative subset, 60

semigroup, 13

separated filtration, 55

sieve, 46

simplicial endofunctor, 35

site, 46

subobject, 4

symbol map, 56

terminal object, 4

the category of fractions, 12

thick subcategory, 70

unit of adjunction, 34

Watts' theorem, 52

Zariski topology, 47

zero object, 4 